\title{
High frequency oscillatory flows in a slightly rarefied gas according to the Boltzmann-BGK equation
}

\author{
Jason Nassios ${ }^{1}$ and John E. Sader ${ }^{1,2, \dagger}$ \\ ${ }^{1}$ Department of Mathematics and Statistics, The University of Melbourne, Victoria 3010, Australia \\ ${ }^{2}$ Kavli Nanoscience Institute and Department of Physics, California Institute of Technology, Pasadena, \\ CA 91125, USA
}

(Received 17 December 2012; revised 24 April 2013; accepted 27 May 2013; first published online 19 July 2013)

The Boltzmann equation provides a rigorous theoretical framework to study dilute gas flows at arbitrary degrees of rarefaction. Asymptotic methods have been applied to steady flows, enabling the development of analytical formulae. For unsteady (oscillatory) flows, two important limits have been studied: (i) at low oscillation frequency and small mean free path, slip models have been derived; and (ii) at high oscillation frequency and large mean free path, the leading-order dynamics are free-molecular. In this article, the complementary case of small mean free path and high oscillation frequency is examined in detail. All walls are solid and of arbitrary smooth shape. We perform a matched asymptotic expansion of the unsteady linearized Boltzmann-BGK equation in the small parameter $v / \omega$, where $v$ is the collision frequency of gas particles and $\omega$ is the characteristic oscillation frequency of the flow. Critically, an algebraic expression is derived for the perturbed mass distribution function throughout the bulk of the gas away from any walls, at all orders in the frequency ratio $v / \omega$. This is supplemented by a boundary layer correction defined by a set of first-order differential equations. This system is solved explicitly and in complete generality. We thus provide analytical expressions up to first order in the frequency ratio, for the density, temperature, mean velocity and stress tensor of the gas, in terms of the temperature and mean velocity of the wall, and the applied body force. In stark contrast to other asymptotic regimes, these explicit formulae eliminate the need to solve a differential equation for a body of arbitrary geometry. To illustrate the utility of these results, we study the oscillatory thermal creep problem for which we find a tangential boundary layer flow arises at first order in the frequency ratio.

Key words: kinetic theory, micro-/nano-fluid dynamics, rarefied gas flow

\section{Introduction}

The Boltzmann equation is a conservation law for the number density of particles in a dilute gas system and has been applied across a range of disciplines. These include applications in plasma physics (Bittencourt 2004), cosmology and astrophysics (Dodelson 2003; Camenzind 2007), molecular biology (Dubois, Ouanounou \& 
Rouzaire-Dubois 2009), and economics (Toscani 2009). When applied to study of the dynamics of a dilute gas, the underlying assumptions and physical basis of the Boltzmann equation ensure solutions remain accurate for flow regimes outside the continuum limit (Grad 1958; Vincenti \& Kruger 1965; Cercignani 1988; Sone 2000). The distinction between continuum and non-continuum flows is most commonly made through use of the Knudsen number $K n$, which is defined as

$$
K n=\frac{\lambda}{L},
$$

where $\lambda$ is the mean free path and $L$ is a characteristic geometric length scale of the flow. Importantly, solutions of the Boltzmann equation provide an accurate model of the (true) flow across the full range of Knudsen number for a dilute gas (Agarwal, Yun \& Balakrishnan 2001; Hadjiconstantinou 2005b). For consistency, in this article we adopt the convention that the constituent molecules/particles of the dilute gas are simply referred to as particles.

Analytical solutions of the Boltzmann equation are difficult to obtain due to the complicated nature of the collision integral, which constrains the efficacy of this approach. The collision integral is derived by considering the number of replenishing and depleting collisions that occur between gas particles in phase space, which results in a quadratic nonlinearity in the mass distribution function for a dilute gas; see Vincenti \& Kruger (1965) and Sone (2007). The Boltzmann equation is therefore a nonlinear integro-partial differential equation for the mass distribution function of the gas. This statement is true for all non-zero gas particle interaction potentials, as discussed by Sone (2007).

For this reason, numerical methods based on the direct simulation Monte Carlo (DSMC) algorithm by Bird (1963) have been developed to directly study flows in complex geometries (Bird 1998). Until recently, DSMC simulations of low Mach number flows were complicated by excessive statistical noise (Hadjiconstantinou et al. 2003; Baker \& Hadjiconstantinou 2005; Chun \& Koch 2005). This issue was addressed by Homolle \& Hadjiconstantinou (2007), who developed the low variance DSMC (LVDSMC) method to solve the hard-sphere Boltzmann equation. Later work by Wagner (2008) and Radtke, Hadjiconstantinou \& Wagner (2011) generalized this approach to simulate low Mach number flows using the variable hard sphere (VHS) collision model.

Model equations have also been proposed to simplify the nature of the collision integral and preserve the qualitative behaviour of the flow (Bhatnagar, Gross \& Krook 1954; Welander 1954; Lebowitz, Frisch \& Helfand 1960; Holway 1963; Bouchut \& Perthame 1993; Struchtrup 1997; Cercignani 2000; Sone 2000). The BGK collision model by Bhatnagar et al. (1954) and Welander (1954) approximates the collision integral with a simple relaxation process. The relaxation rate is the interparticle collision frequency, $v$, of the gas, which is independent of particle velocity. Importantly, the physical conservation laws and the Boltzmann $H$-theorem also hold for the Boltzmann-BGK equation (Sone 2000). The insight afforded by this model equation is sufficient to describe the qualitative properties of the (true) flow in many cases of practical interest (Vincenti \& Kruger 1965; Cercignani 2000; Sone 2007). Furthermore, the DSMC and LVDSMC methods discussed for the Boltzmann equation have been applied to study of the Boltzmann-BGK equation (Ramanathan \& Koch 2009; Hadjiconstantinou, Radtke \& Baker 2010). Other numerical schemes, such as finite differencing techniques and the lattice Boltzmann (LB) method, have been used to investigate a number of canonical gas flows (Loyalka, Petrellis \& Storvick 1979; 
Chen et al. 2003; Yu, Girimaji \& Luo 2005; Loyalka \& Tompson 2009; Shi \& Sader 2010; Yap \& Sader 2012). Analytical moment methods have also been proposed and applied; see Struchtrup \& Torrilhon (2003), Torrilhon \& Struchtrup (2008) and Taheri et al. (2009).

Two well-documented shortcomings of the Boltzmann-BGK equation exist. First, the simplified collision integral depends on the local equilibrium Maxwellian distribution function, and is thus highly nonlinear (Cercignani 2000; Sone 2000). Second, the BGK approximation contains a single parameter, the inter-particle collision frequency. This sole degree of freedom is insufficient to simultaneously set the correct gas viscosity and thermal conductivity. As such, the correct Prandtl number for a monatomic gas is not recovered (Cercignani 2000; Sone 2000).

Alternatives to the BGK kinetic model have been proposed to address these issues. The quadratic nonlinearity of the Boltzmann equation is recovered under certain conditions by the integro-differential model studied by Lebowitz et al. (1960); see also Cercignani (2000). The correct Prandtl number is predicted by the ellipsoid statistical (ES) model proposed by Holway (1963), where the local equilibrium Maxwellian solution that appears in the BGK approximation is replaced by an anisotropic Gaussian defined in terms of the Prandtl number and the stress tensor of the gas (Holway 1966; Cercignani 2000; Brull \& Schneider 2008; Gallis \& Torczynski 2011). The BGK approximation is a special case of the ES model, that is recovered when the Prandtl number is set to unity. Relative to the BGK approximation, the improved accuracy that these model equations and others offer comes at a cost of diminished tractability (Cercignani 1988, 2000). Further, the physical intuition garnered by an analysis of the Boltzmann-BGK equation has proved to be important in many theoretical investigations (Sone 2000). As such, this article focuses on the Boltzmann-BGK equation.

Asymptotic methods have been utilized to investigate steady flow according to both the Boltzmann equation for hard spheres and the Boltzmann-BGK equation in the small and large Knudsen number limits, i.e. $K n \ll 1$ and $K n \gg 1$ (Hilbert 1900; Knudsen 1909a,b; Hilbert 1912; Chapman 1916; Enskog 1917). Hilbert (1900, 1912) performed an expansion of the Boltzmann equation in the Knudsen number, and recovered the Euler equations to leading order; higher-order equations in the Knudsen number contained additional forcing terms, but the classical nonlinear Navier-Stokes equations did not appear (Sone 2000). Sone $(1969,1974)$ generalized the approach of Hilbert in an analysis of the linearized Boltzmann-BGK equation for steady, slightly rarefied gas flows across solid walls, i.e. $K n \ll 1$. A matched asymptotic expansion was performed, where solutions for the perturbation to the mass distribution function and its respective moments were derived in the asymptotic limit of infinitesimal scaled Knudsen number $k$, defined as

$$
k=\frac{\sqrt{\pi}}{2} K n .
$$

The method implemented by Sone formally accounted for the thin Knudsen layer present near the wall at small Knudsen numbers, which is not modelled by classical continuum theory. The usual Stokes equations for creeping flow were derived in the bulk (outer) flow region, while analysis of the inner region yielded a general set of slip models and Knudsen layer corrections. The techniques and results employed by Sone have been applied to the study of many canonical rarefied gas flows (Cercignani 1962, 1964; Sone 1966, 1969, 1972, 1974). The hard-sphere Boltzmann equation was 
investigated much later (Ohwada, Sone \& Aoki 1989; Sone, Ohwada \& Aoki 1989; Ohwada \& Sone 1992).

Critically, the steady-state assumption underlying these analyses is brought into question in modern nanoscale flows, which exhibit Knudsen numbers of order unity, and are distinctly oscillatory (time-varying) with a broad oscillation frequency range, $\omega$. For example, oscillation frequencies of several hundred megahertz have been reported for nanoelectromechanical systems (NEMS) (Bargatin, Kozinsky \& Roukes 2007), while oscillation frequencies ranging from tens of gigahertz to several terahertz have been observed for metal nanoparticles (Pelton et al. 2009; Juvé et al. 2010). This broad spectrum of operating conditions encompasses the inter-particle collision frequency, $v$, of many typical gases at standard laboratory conditions. In the low frequency range, gas particles undergo many inter-particle collisions over a single oscillation period; this contrasts with the high frequency range, where $\omega$ is much larger than the relaxation frequency of the gas $v$. To characterize this behaviour, the frequency ratio, $\theta$, is defined:

$$
\theta=\frac{\omega}{v}
$$

The limit $\theta \ll 1$ therefore corresponds to the low oscillation frequency range, while $\theta \gg 1$ corresponds to high frequency. The purpose of this article is to theoretically investigate the dynamics of oscillatory gas flows in the high frequency limit, based on an analysis of the Boltzmann-BGK equation.

The approach of Sone $(1969,1974)$ was revisited recently by Nassios \& Sader (2012), who examined the linearized Boltzmann-BGK equation for oscillatory gas flows. In independent work, Takata \& Hattori (2012) presented an analysis of the linearized Boltzmann equation for hard spheres, and the linearized Boltzmann-BGK equation. Both studies derived slip models and bulk flow hydrodynamic equations for flows at low frequency in a slightly rarefied gas, i.e. $\theta \ll 1$ and $k \ll 1$. The low frequency assumption was implicit in both cases. In Nassios \& Sader (2012), $\theta$ is written in the form

$$
\theta=\frac{1}{2} \beta k^{2},
$$

where $\beta=\omega L^{2} / v_{\text {Kin }}$ is the Stokes number and $v_{\text {Kin }}$ is the kinematic viscosity. The frequency ratio $\theta$ is small when operating in the asymptotic limit of slight gas rarefaction $k \ll 1$, for all values of inertia $\beta$. Takata \& Hattori (2012) express the frequency ratio as

$$
\theta=S t k
$$

where the Strouhal number

$$
S t=\frac{\omega L}{v_{m p}\left(T_{0}\right)}
$$

is assumed to be $O(k)$. The most probable speed of gas particles at the equilibrium temperature $T_{0}$ is $v_{m p}\left(T_{0}\right)$. Interestingly, in both studies the classical steady slip models for the tangential component of the mean velocity remained unaltered at second order in the scaled Knudsen number $k$, while all other slip models, i.e. for the temperature and normal component of the mean velocity, were modified. In contrast, a variation of the classical linearized Navier-Stokes equation for the bulk flow appeared at first order in the scaled Knudsen number for non-isothermal systems - an additional body force term appeared, that was proportional to $\beta$. Therefore, the hydrodynamic equations are 
modified by unsteadiness at $O(k)$, whereas the slip boundary conditions are altered at $O\left(k^{2}\right)$. Nassios \& Sader (2012) explored the effect of the additional body force at $O(k)$, by studying the oscillatory (time-varying) thermal creep problem, where temperature gradients are applied along two adjacent walls.

Unsteady effects in the complementary ultra-rarefied limit $K n \gg 1$ have also been investigated using asymptotic methods, by Sone (2007). No assumption was made on the form of the collision integral, with the results applicable for all interaction potentials and model equations. To facilitate analysis, the frequency ratio was rewritten as in (1.5), and it was assumed that $S t \sim O(1)$. As such, the frequency ratio was assumed to be large, $\theta \gg 1$ (see (1.5)), and an asymptotic expansion was performed in the inverse of the scaled Knudsen number $k^{-1}$. No boundary layer flow arose. Instead, in the asymptotic limit of ultra-rarefied and highly oscillatory flow, i.e. $k^{-1} \rightarrow 0$ and $\theta^{-1} \rightarrow 0$, the mass distribution function throughout the bulk of the gas was given by the solution of the collisionless Boltzmann equation, as expected; see Clausing (1971), Sone (1984), Cercignani (2000), Park, Bahukudumbi \& Beskok (2004), Hadjiconstantinou (2005a), Sharipov \& Kalempa (2007) and Yap \& Sader (2012). The equations for the higher-order terms in the $k^{-1}$-expansion of the mass distribution function took a similar form. However, source terms in these equations appeared that account for the effect of inter-particle collisions. The general solution to the first-order correction for the mass distribution function in the inverse scaled Knudsen number $k^{-1}$ was found by the method of characteristics. This correction was defined in terms of the leading-order solution, and the task of solving the collisionless Boltzmann equation remained.

The purpose of this article is to present a formal asymptotic analysis of the Boltzmann-BGK equation for slightly rarefied oscillatory flows at high oscillation frequency, i.e. small Knudsen numbers and large frequency ratios $(k \ll 1$ and $\theta \gg 1)$. In so doing, we elucidate the physical distinction between this class of highly oscillatory flows and the ultra-rarefied limit $(k \gg 1)$ investigated by Sone (2007). Our work also provides the complementary high frequency solution to the recent studies by Nassios \& Sader (2012) and Takata \& Hattori (2012), of slightly rarefied flows at low frequency $(\theta \ll 1)$. These two analyses can be realized physically using a single oscillating device of fixed (small) size, operating at low and high frequency. An asymptotic expansion in the inverse frequency ratio $\theta^{-1}$ is performed, and $k$ is assumed to be a small parameter. As we shall show, flow throughout the bulk of the gas (away from any walls) is not free-molecular. This contrasts with the ultra-rarefied formulation of Sone (2007), where flow of gas particles is free-molecular to leading order in $k^{-1}$ throughout the gas. Instead, the mass distribution function away from the walls for slightly rarefied flow up to first order in $\theta^{-1}$ is directly proportional to the applied oscillatory body force; the linearized inviscid Euler equation thus describes the mean velocity of the bulk flow away from the walls to leading order in $\theta^{-1}$. The first-order corrections are also derived and discussed.

A matched asymptotic expansion is performed to account for the collisionless boundary layer near the walls. The dominant wall-normal length scale is the acoustic length scale $L_{c}=v_{m p} / \omega$, which is small relative to the mean free path of the gas; this acoustic length scale specifies the nominal thickness of the collisionless boundary layer. Free-molecular transport away from the wall, i.e. in the wall-normal direction only, governs the local leading-order correction to the mass distribution function in $\theta^{-1}$. The usual multi-dimensional collisionless Boltzmann equation, which fully describes the leading-order dynamics of an ultra-rarefied gas flow, does not appear in 
the collisionless boundary layer. At higher order, source terms arise in this region that describe the influence of (i) collisions, and (ii) the advection of gas particles parallel to the wall; these effects become increasingly significant at small inverse frequency ratios.

Importantly, the boundary layer equations can be solved in complete generality. We thus derive explicit and general analytical expressions for the gas density, temperature, mean velocity and stress tensor components, up to $O\left(\theta^{-1}\right)$. Critically, these formulae supplant the need to solve any additional differential equations for the bulk flow quantities. This explicit solvability property contrasts to slightly rarefied flows at low frequency $(k \ll 1$ and $\theta \ll 1)$, and ultra-rarefied flows at high frequency $(k \gg 1$ and $\theta \gg 1$ ); see Sone (2007) and Nassios \& Sader (2012) and Takata \& Hattori (2012).

In line with previous work, application of this theory is illustrated with a study of the oscillatory (time-varying) thermal creep flow generated between two adjacent walls. As a cornerstone rarefaction phenomenon, this mechanism has been widely discussed and applied. Thermal creep was first studied by Maxwell (1879), and is the dominant physical effect driving steady flow in the Knudsen compressor (Knudsen 1909b, 1910; Loyalka 1971; Vargo et al. 1999; Sone 2007). More recently, steady thermal creep has also been used to explain motion of (volatile) Leidenfrost drops on a ratchet surface; see Lagubeau et al. (2011) and Würger (2011).

An analytical formula for the classical steady thermal creep flow at small scaled Knudsen number $k$ was derived by Sone (1966) using the linearized Boltzmann-BGK equation. The single-wall problem was considered as opposed to the two-wall case; this yields an integral equation for the mean tangential velocity of the gas that is of identical form to the one studied by Tamada \& Sone (1966). Using the general solution given there, it was shown that the leading-order thermal creep flow was $O(k)$; because the flow was steady, this result was also valid in the two-wall case studied numerically by Loyalka et al. (1979), where identical (symmetric) temperature gradients are applied along a pair of parallel, plane walls. The ultra-rarefied limit was also investigated by Sone (1984), who showed that no steady thermal creep flow occurs if the gas is free-molecular, i.e. $k^{-1}=0$. This result was proved for the Maxwell-type boundary condition, of which diffuse reflection is one special case; see Sone (1985, 2000). Preziosi (1992) later studied the thermal creep problem for a dense gas using the discrete Boltzmann equation, where both binary and triple inter-particle collisions are permitted to occur. As discussed, the oscillatory (time-varying) problem was studied for low frequency ratio and small scaled Knudsen number by Nassios \& Sader (2012). For zero inertia, the classical steady result at small Knudsen number of Sone (1966) was recovered; additional out-of-phase corrections to the bulk flow appeared as inertia increased.

While oscillatory shear-driven flows, e.g. oscillatory Couette flow, have been widely studied using a variety of numerical and analytical techniques, these flows are unidirectional in the linear limit (Park et al. 2004; Hadjiconstantinou 2005a; Sharipov \& Kalempa 2007, 2008; Tang et al. 2008; Yap \& Sader 2012). Small collisional corrections arise at $O\left(\theta^{-1}\right)$, but these do not alter the leading-order (free-molecular) shear flow. For this reason, we only present a detailed exposition of the oscillatory (time-varying) thermal creep problem in $\S 4$.

As we shall show for $\theta \gg 1$ and $k \ll 1$, flow driven by oscillatory temperature gradients along two parallel plane walls is confined to thin collisionless boundary layers - unsteady effects do not result in a bulk inviscid flow away from the walls. Analysis of the boundary layer flows near each wall decouple, and can be performed independently. We therefore consider the related problem of a single wall, and seek the 
thermal creep flow generated in a half-space of gas. No thermal creep flow is found to leading order in the inverse frequency ratio $\theta^{-1}$, i.e. no mean flow occurs parallel to the wall, but a normal flow away from the wall is observed. A thermal creep flow does develop at first order in the inverse frequency ratio, i.e. $O\left(\theta^{-1}\right)$, and the physical mechanisms driving this flow are explored. The complete solution to first order in $\theta^{-1}$ is therefore critical in describing the full two-dimensional flow that arises for $k \ll 1$ and $\theta \gg 1$.

The outline of this article is as follows. In $\S 2$, a brief summary of the linearization of the governing equation is presented, assuming small perturbations about the equilibrium gas state. We assume the flow to be slightly rarefied and of high frequency, i.e. $k \ll 1$ and $\theta \gg 1$. The appropriate scaling analysis for this problem is discussed in $\S 2.1$. The shape of the wall is arbitrary, and is characterized by the method of moving frame; see $\S 2.2$. The boundary conditions are given in $\S 2.3$. In $\S 3$ we provide the details of our matched asymptotic expansion for the mass distribution function and its moments. Explicit analytical formulae for all bulk flow hydrodynamic quantities are given in table 1. The leading-order collisionless boundary layer corrections are given in table 2, while the first-order corrections appear in appendix A. Finally, the oscillatory thermal creep problem is investigated in $\S 4$. Details of the mathematical and numerical analysis are relegated to appendices B and C.

\section{Formalism}

We assume that the mass distribution function $F(\boldsymbol{x}, \boldsymbol{v}, t)$ of the homogeneous, dilute gas obeys the Boltzmann-BGK equation (Bhatnagar et al. 1954; Welander 1954),

$$
\frac{\partial F}{\partial t}+v_{i} \frac{\partial F}{\partial x_{i}}+a_{i} \frac{\partial F}{\partial v_{i}}=v\left(\rho(\boldsymbol{x}, t) f_{0}(\boldsymbol{v})-F\right),
$$

where $t$ is time, $v$ is the inter-particle collision frequency, and $\boldsymbol{x}, \boldsymbol{v}$ and $\boldsymbol{a}$ are the particle position, particle velocity and body force, respectively. The local gas density is $\rho(\boldsymbol{x}, t)$, while the equilibrium velocity distribution function $f_{0}(\boldsymbol{v})$ is evaluated at the local gas temperature $T(\boldsymbol{x}, t)$ and mean velocity $\overline{\boldsymbol{v}}(\boldsymbol{x}, t)$, and takes the Gaussian form

$$
f_{0}(\boldsymbol{v})=\left(\frac{1}{\sqrt{\pi} v_{m p}(T)}\right)^{3} \exp \left(-\left[\frac{v_{i}-\bar{v}_{i}}{v_{m p}(T)}\right]^{2}\right) .
$$

Here, $k_{B}$ is Boltzmann's constant and the most probable speed of gas particles of mass $m$ at temperature $T(\boldsymbol{x}, t)$ is

$$
v_{m p}(T)=\sqrt{\frac{2 k_{B} T}{m}} .
$$

The local gas density $\rho$, mean gas velocity $\overline{\boldsymbol{v}}$, gas temperature $T$ and stress tensor $e_{i j}$ are given by the following moments of the mass distribution function:

$$
\begin{gathered}
\rho=\int_{-\infty}^{\infty} F \mathrm{~d} \boldsymbol{v}, \\
\overline{\boldsymbol{v}}=\frac{1}{\rho} \int_{-\infty}^{\infty} \boldsymbol{v} F \mathrm{~d} \boldsymbol{v}, \\
\frac{3 k_{B} T}{m}=\frac{1}{\rho} \int_{-\infty}^{\infty}(\boldsymbol{v}-\overline{\boldsymbol{v}})^{2} F \mathrm{~d} \boldsymbol{v},
\end{gathered}
$$




$$
\begin{gathered}
e_{i j}=\int_{-\infty}^{\infty}\left(v_{i}-\bar{v}_{i}\right)\left(v_{j}-\bar{v}_{j}\right) F \mathrm{~d} \boldsymbol{v}, \\
\frac{p}{\rho}=\frac{k_{B} T}{m},
\end{gathered}
$$

where the equation of state is the ideal gas law, and $p(x, t)$ is the local pressure of the gas.

We consider weakly perturbed solutions of (2.1) for which deviations in the mass distribution function and its moments about their equilibrium states are small. This corresponds to an assumption of low Mach and Reynolds number flow, i.e. $M a \ll R e \ll 1$. The gas is therefore slightly rarefied, which can be shown using the von Kármán (1963) relation:

$$
K n \propto \frac{M a}{R e} \ll 1 .
$$

The mass distribution function $F$ and its moments are thus written as

$$
\begin{gathered}
\rho=\rho_{0}(1+\sigma(\boldsymbol{x}, t)), \\
T=T_{0}(1+\tau(\boldsymbol{x}, t)), \\
p=p_{0}(1+P(\boldsymbol{x}, t)), \\
e_{i j}=p_{0}\left(\delta_{i j}+\epsilon_{i j}\right), \\
F=\rho_{0} E_{0}(1+\phi(\boldsymbol{x}, \boldsymbol{v}, t)) .
\end{gathered}
$$

The functions $\rho_{0}, T_{0}, p_{0}$ and $\rho_{0} E_{0}$ are the equilibrium density, temperature, pressure and mass distribution function, respectively, while $\sigma, \tau, P$ and $\phi$ are perturbations to these equilibrium values, with $\epsilon_{i j}$ defined as the perturbation to the stress tensor. $E_{0}$ in (2.6) is given by

$$
E_{0}=\left(\frac{1}{\sqrt{\pi} v_{m p}\left(T_{0}\right)}\right)^{3} \exp \left(-\left[\frac{v_{i}}{v_{m p}\left(T_{0}\right)}\right]^{2}\right) .
$$

We then substitute (2.6) into (2.1), and retain terms linear in the perturbed quantities $\sigma$, $\tau, P$ and $\phi$. This allows all time-varying functions to be expressed as

$$
\alpha(\boldsymbol{x}, \boldsymbol{v}, t)=\tilde{\alpha}(\boldsymbol{x}, \boldsymbol{v}) \exp (-\mathrm{i} \omega t),
$$

where $\mathrm{i}$ is the usual imaginary unit, and $\alpha$ represents any of (i) the perturbed quantities defined in (2.6), (ii) the mean gas velocity, $\bar{v}_{i}$, or (iii) the body force $a_{i}$, which is regarded in general as oscillatory (time-varying). For simplicity, the " , notation is omitted henceforth, and we operate exclusively in the frequency domain. This yields the linearized Boltzmann-BGK equation for oscillatory flow,

$$
-\mathrm{i} \theta \phi+\frac{1}{v} v_{i} \frac{\partial \phi}{\partial x_{i}}-\frac{2}{v v_{m p}^{2}\left(T_{0}\right)} v_{i} a_{i}=\sigma-\phi+\frac{2}{v_{m p}^{2}\left(T_{0}\right)} \bar{v}_{i} v_{i}+\left[\left(\frac{v_{i}}{v_{m p}\left(T_{0}\right)}\right)^{2}-\frac{3}{2}\right] \tau,
$$

where we have used the definition of $\theta$ in (1.3) for the frequency ratio. Note that the linearization procedure removes all gradients of $\phi$ with respect to the particle velocity $v_{i}$, given that $a_{i}$ is regarded to be oscillatory. Performing a similar procedure on (2.4) 
yields the linearized moment relations

$$
\begin{gathered}
\sigma=\int_{-\infty}^{\infty} \phi E_{0} \mathrm{~d} v \\
\bar{v}_{i}=\int_{-\infty}^{\infty} v_{\mathrm{i}} \phi E_{0} \mathrm{~d} v \\
\frac{3}{2} \tau=\int_{-\infty}^{\infty}\left(\left(\frac{v_{i}}{v_{m p}\left(T_{0}\right)}\right)^{2}-\frac{3}{2}\right) \phi E_{0} \mathrm{~d} v \\
\frac{v_{m p}^{2}\left(T_{0}\right)}{2} \epsilon_{i j}=\int_{-\infty}^{\infty} v_{i} v_{j} \phi E_{0} \mathrm{~d} v \\
P=\sigma+\tau .
\end{gathered}
$$

\subsection{Scaling analysis}

We choose the following scalings for the particle velocity $v_{i}$, mean velocity $\bar{v}_{i}$ and body force $a_{i}$ :

$$
v_{s}=v_{m p}\left(T_{0}\right), \quad \bar{v}_{s}=v_{m p}\left(T_{0}\right), \quad a_{s}=v_{m p}\left(T_{0}\right) \omega,
$$

where a subscript ' $s$ ' denotes a scale. The choice of $a_{s}$ is appropriate under the assumption of an oscillatory body force.

Three distinct length scales exist: (i) the characteristic geometric length scale of the solid boundary/wall, $L$, (ii) the gas mean free path, $\lambda$, and (iii) the acoustic length scale, $L_{c}$, which is defined as

$$
L_{c}=\frac{v_{m p}\left(T_{0}\right)}{\omega} .
$$

This is the most probable distance travelled by a gas particle in equilibrium, over a single oscillation period.

As discussed in $\S 1$, the unsteady flow of an ultra-rarefied gas $k \gg 1$ was investigated by Sone (2007), where the Strouhal number defined in (1.6) was assumed to be of order unity, i.e. $S t \sim O(1)$. For this choice of $k$ and $S t$, the inverse frequency ratio $\theta^{-1}$ is small; see (1.5). By (1.5) and (1.6) the three length scales in the analysis of Sone (2007) are then related by the inequality

$$
\lambda \gg L \sim L_{c} \text {. }
$$

To study the bulk flow of the gas (away from any wall), all spatial dimensions are scaled isotropically by the characteristic geometric length scale L. From (2.13), the mean free path is much larger than $L$ and the bulk flow is thus free-molecular in all spatial directions for this limit of $k \gg 1$, as expected. In addition, $L$ and $L_{c}$ are identical given that $S t \sim O(1)$. As such, the free-molecular solution remains valid for $k \gg 1$ near any solid wall. No boundary layer flow occurs, and the mass distribution function is obtained via a Hilbert expansion in the small parameter $k^{-1}$, which is matched directly to the boundary condition at the wall at all orders in $k^{-1}$.

In this article, we solve the linearized Boltzmann-BGK equation in (2.9) to study high frequency oscillatory flows when the gas is slightly rarefied, i.e. $\theta \gg 1$ and $k \ll 1$. This provides the complementary analysis to Sone (2007), who considered $k \gg 1$ only. For $\theta \gg 1$ and $k \ll 1,(1.2)$ and (1.3) yield the following relationship between the 


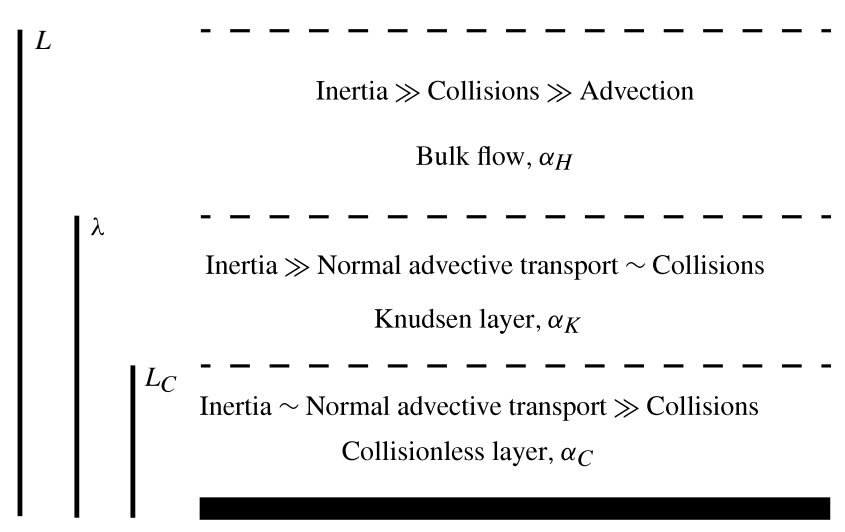

FIGURE 1. An illustration of the general physical structure of the flow at high frequency in a slightly rarefied gas, i.e. $\theta \gg 1$ and $k \ll 1$. The illustrated upper, middle and lower regions are referred to as the Hilbert (bulk flow), Knudsen and collisionless regions, respectively.

three length scales:

$$
L_{c} \ll \frac{\sqrt{\pi}}{2} \lambda \ll L .
$$

This breaks the degeneracy between $L$ and $L_{c}$ that exists in the ultra-rarefied limit of $k \gg 1$ studied by Sone (2007), and in general yields three distinct flow regimes; see figure 1. We now discuss these three regions in detail, which are formally obtained by balancing terms in the linearized Boltzmann-BGK equation in (2.9).

\subsubsection{Hilbert region}

The characteristic geometric length scale $L$ in the Hilbert region is found to be large relative to all other length scales of the flow; see figure 1 and analysis below. Therefore, flow throughout the bulk of the gas away from the walls is not freemolecular - this differs fundamentally from the $k \gg 1$ limit. Under the assumption of small Knudsen number, inter-particle collisions dominate gas particle advection. This result is also true for slightly rarefied flows at low oscillation frequencies $\theta \ll 1$; see Nassios \& Sader (2012) and Takata \& Hattori (2012), where collisional effects also dominate inertia. However, at high oscillation frequency ratios $(\theta \gg 1)$, collisional effects are negligible relative to inertia.

For a general length scale $l$, the method of dominant balance may be used to show that the advection term in (2.9) is negligible relative to all other terms provided that

$$
l \gg \frac{v_{m p}\left(T_{0}\right)}{v}=\frac{\sqrt{\pi}}{2} \lambda .
$$

By (2.14), the length scale $l$ in the bulk flow region is defined to be $l \equiv L$. Therefore, the inertial terms in (2.9) are $O(\theta)$, while the collisional terms on the right-hand side are $O(1)$. The advection term is $O(k)$, which is small in comparison. We thus investigate the bulk flow by scaling the linearized Boltzmann-BGK equation isotropically by $L$. A Hilbert expansion is performed in the small parameter $\theta^{-1}$ to derive the relevant hydrodynamic equations. 


\subsubsection{Knudsen region}

For small scaled Knudsen numbers $k \ll 1$, the effect of gas rarefaction is localized near the wall within a thin boundary layer referred to as the Knudsen layer; the thickness of this layer scales with the mean free path $\lambda$, as shown in figure 1 . Within the Knudsen layer, advection of reflected gas particles away from the wall, i.e. in the wall-normal direction only, locally balances the effect of inter-particle collisions on the flow; see Sone (1969, 1974, 2000), Nassios \& Sader (2012) and Takata \& Hattori (2012). Mathematically, the wall-normal component of the advective term in (2.9) is equivalent in order to the collisional contributions on the right-hand side. This balance directly gives the appropriate length scale $l$ for this region:

$$
l=\frac{\sqrt{\pi} \lambda}{2},
$$

where $\sqrt{\pi} / 2 \sim O(1)$.

Both the thickness of the Knudsen layer and the dominant wall-normal length scale therefore scale with the mean free path. As such, to study flow in the Knudsen layer the linearized Boltzmann-BGK equation is rescaled in the wall-normal direction by $l$; see (2.16). As we discussed, only advection normal to the wall balances collisional effects at small Knudsen numbers. Therefore, the tangential directions remain scaled by $L$, as before.

\subsubsection{Collisionless region}

Throughout both the bulk of the gas and the Knudsen layer, inertial effects dominate the flow due to the high oscillation frequency. However, since

$$
\theta=\frac{\sqrt{\pi}}{2} \frac{\lambda}{L_{c}},
$$

high frequency flow, i.e. $\theta \gg 1$, corresponds to the case where the acoustic wavelength $L_{c}$ is much smaller than the mean free path, $\lambda$, i.e. $L_{c} \ll \lambda$. Particles undergoing diffuse reflection from the wall may therefore travel a short distance $L_{c} \ll \lambda$ away from the wall in a single oscillation period. Physically, this corresponds to a (local) balance between the advection of reflected gas particles away from the wall, i.e. in the wall-normal direction only, and particle inertia; see figure 1, which illustrates this thin collisionless layer that forms near the wall of thickness $L_{c}$. Mathematically, the wall-normal component of the advective term in (2.9) locally balances the remaining inertial terms on the left-hand side of this equation. The method of dominant balance then gives the appropriate length scale $l$ in the wall-normal direction,

$$
l \sim \frac{v_{m p}\left(T_{0}\right)}{\omega}=L_{c} .
$$

As we shall show, this balance corresponds to a leading-order local flow of gas particles in the normal direction from the wall, that is free-molecular. We study the flow in this collisionless layer by rescaling (2.9) once more in the wall-normal direction by the local length scale $L_{c}$; see figure 1 . As discussed for the Knudsen layer flow, the tangential directions again remain scaled by the macroscopic length scale $L$.

\subsubsection{Mathematical approach}

To account for this three-layer structure, we seek a solution for $\phi$ and all its respective moments (all represented by $\alpha$ ) using a 3-way matched asymptotic expansion in the (small) inverse frequency ratio $\theta^{-1} \ll 1$. Importantly, expanding in 
small $\theta^{-1}$ first, rather than small scaled Knudsen number $k$, ensures that the acoustic length scale $L_{c}$ approaches zero relative to the mean free path $\lambda$, for all small $k$. The resulting composite solution for the flow is thus:

$$
\alpha=\alpha_{H}+\alpha_{K}+\alpha_{C}
$$

where the bulk flow quantities in the Hilbert region are represented by a subscript $H$, while the Knudsen layer corrections are denoted by a subscript $K$. The collisionless layer corrections are distinguished by a subscript $C$. The sum of the three distinct components yields the complete solution; see (2.19). These three components and the thickness of the two boundary layers are illustrated in figure 1.

\subsection{Generalized coordinates}

The surface geometry within the Knudsen and collisionless layers is specified using the method of moving frame (Cartan 1977; Sone 2000). A local orthonormal coordinate system is chosen comprising two (principal) tangent vectors $t_{i}^{1}$ and $t_{i}^{2}$ (parametrized by $\chi_{1}$ and $\chi_{2}$ respectively) and an associated outward wall-normal $n_{i}$. The coordinate normal to a solid wall is denoted by $\eta_{K}$ within the Knudsen layer, and is scaled by the mean free path $\lambda$. Within the collisionless layer, this coordinate is denoted by $\eta_{C}$ and is scaled by the most probable distance travelled $L_{c}$. The wall curvature is chosen to be negative when its centre of curvature lies within the gas (Sone 1969, 1974). The analysis of the Knudsen and collisionless layers requires the following geometric relations (Cartan 1977; Sone 2000):

$$
\begin{gathered}
t_{j}^{q} \frac{\partial t_{i}^{q}}{\partial x_{j}}=-\kappa_{q} n_{i}-(-1)^{q} g_{q} t_{i}^{3-q}, \\
t_{j}^{q} \frac{\partial t_{i}^{3-q}}{\partial x_{j}}=(-1)^{q} g_{q} t_{i}^{q}, \\
t_{j}^{q} \frac{\partial n_{i}}{\partial x_{j}}=\kappa_{q} t_{i}^{q}, \\
\kappa_{i j}=\kappa_{1} t_{i}^{1} t_{j}^{1}+\kappa_{2} t_{i}^{2} t_{j}^{2}, \\
\bar{\kappa}=\frac{\kappa_{1}+\kappa_{2}}{2},
\end{gathered}
$$

where the index $q$ takes the values 1,2 , and the normal and geodesic curvatures are $\kappa_{q}$ and $g_{q}$, respectively. The curvature tensor $\kappa_{i j}$ is defined on the surface in terms of the normal curvatures and local tangents, whereas the mean curvature is $\bar{\kappa}$.

\subsection{Boundary conditions}

In this section, we discuss solution of (2.9) subject to classic diffuse reflection from a rigid wall of arbitrary and smooth shape. Without loss of generality, we assume the presence of a single wall, and choose a local coordinate system as per the formalism in $\S 2.2$ to analyse the Knudsen and collisionless layer flows; see figure 1 . The results generalize to multiple walls by choosing a unique local coordinate system to study the local flows near each wall.

We assume the following functional form of the mass distribution function for reflected particles $\left(v_{i} n_{i} \geqslant V_{i} n_{i}\right)$ :

$$
F_{b}=\rho_{b}\left(\frac{1}{\sqrt{\pi} v_{m p}\left(T_{b}\right)}\right)^{3} \exp \left(-\left[\frac{v_{i}-V_{i}}{v_{m p}\left(T_{b}\right)}\right]^{2}\right),
$$


where the subscript ' $b$ ' denotes values at the solid wall, and $V_{i}$ is the velocity of the solid wall.

Zero net mass flux at the solid wall is also required. This immediately leads to the following results for the density of particles reflected from the wall and the usual no-penetration condition,

$$
\begin{gathered}
\rho_{b}=-2 \frac{\sqrt{\pi}}{v_{m p}\left(T_{b}\right)} \int_{\left(v_{i}-V_{i}\right) n_{i}<0}\left(v_{i}-V_{i}\right) n_{i} F \mathrm{~d} \boldsymbol{v}, \\
V_{i} n_{i}=\bar{v}_{i} n_{i} .
\end{gathered}
$$

Linearizing and scaling (2.21) and (2.22) yields the required set of boundary conditions for particles reflected from the solid wall,

$$
\begin{gathered}
\phi_{b}=\sigma_{b}+2 V_{i} v_{i}+\left(v_{i}^{2}-\frac{3}{2}\right) \tau_{b} \\
\sigma_{b}=\sqrt{\pi} V_{i} n_{i}-\frac{1}{2} \tau_{b}-2 \sqrt{\pi} \int_{-\infty}^{\infty} \int_{-\infty}^{\infty} \int_{-\infty}^{0} \xi \phi E\left(v_{i} t_{i}^{1}, v_{i} t_{i}^{2}, \xi\right) \mathrm{d} \xi \mathrm{d}\left(v_{i} t_{i}^{1}\right) \mathrm{d}\left(v_{i} t_{i}^{2}\right) \\
V_{i} n_{i}=\bar{v}_{i} n_{i}, \\
\xi=v_{i} n_{i}-V_{i} n_{i},
\end{gathered}
$$

where $\phi$ in the second line of (2.23) is evaluated at the wall, and we have defined the normalized Gaussian function

$$
E\left(v_{1}, v_{2}, v_{3}\right)=\pi^{-3 / 2} \exp \left(-v_{i}^{2}-v_{2}^{2}-v_{3}^{2}\right) .
$$

From (2.23), it then follows that outgoing particles from the solid wall satisfy the condition

$$
\left.\phi_{C, \xi \geqslant 0}\right|_{\eta_{C}=0}=\phi_{b}-\phi_{H}-\phi_{K}
$$

where $\phi_{H}$ and $\phi_{K}$ in (2.25) are evaluated at the solid wall. In the outer region of the Knudsen and collisionless layers, i.e. $\eta_{K} \rightarrow \infty$ and $\eta_{C} \rightarrow \infty$, respectively, we also require that $\phi_{K}$ and $\phi_{C}$ decay to zero:

$$
\begin{gathered}
\lim _{\eta_{K} \rightarrow \infty} \phi_{K, \xi<0}=0, \\
\lim _{\eta_{C} \rightarrow \infty} \phi_{C, \xi<0}=0 .
\end{gathered}
$$

Finally, the solid wall is considered rigid, as formalized by the condition of zero rate-of-strain:

$$
\frac{\partial V_{i}}{\partial x_{j}}+\frac{\partial V_{j}}{\partial x_{i}}=0
$$

This ensures that the curvatures $\kappa_{s}$ and $g_{s}$ are constant and well-defined over the course of the flow.

\section{Asymptotic results}

The mass distribution function is expanded according to (2.19) and scaled in each flow region (see $§ 2.1$ ). This leads to the following set of scaled governing equations:

$$
-\frac{1}{\theta}\left(\mathrm{i} \phi_{H}+2 a_{i} v_{i}\right)+k v_{i} \frac{\partial \phi_{H}}{\partial x_{i}}=\sigma_{H}-\phi_{H}+2 \bar{v}_{H \mid i} v_{i}+\left(v_{i}^{2}-\frac{3}{2}\right) \tau_{H},
$$




$$
\begin{aligned}
-\frac{\mathrm{i} \phi_{K}}{\theta}= & \sigma_{K}-\phi_{K}+2 \bar{v}_{K \mid i} v_{i}+\left(v_{i}^{2}-\frac{3}{2}\right) \tau_{K}-v_{i} n_{i} \frac{\partial \phi_{K}}{\partial \eta_{K}} \\
& -k\left(v_{i} t_{i}^{1} t_{j}^{1} \frac{\partial \phi_{K}}{\partial x_{j}}+v_{i} t_{i}^{2} t_{j}^{2} \frac{\partial \phi_{K}}{\partial x_{j}}\right) \\
\frac{1}{\theta}\left(-\mathrm{i} \phi_{C}+v_{i} n_{i} \frac{\partial \phi_{C}}{\partial \eta_{C}}\right)= & \sigma_{C}-\phi_{C}+2 \bar{v}_{C \mid i} v_{i}+\left(v_{i}^{2}-\frac{3}{2}\right) \tau_{C} \\
& -k\left(v_{i} t_{i}^{1} t_{j}^{1} \frac{\partial \phi_{C}}{\partial x_{j}}+v_{i} t_{i}^{2} t_{j}^{2} \frac{\partial \phi_{C}}{\partial x_{j}}\right) .
\end{aligned}
$$

Here, we introduce the vertical line ' $\mid$ ' in the subscript to delineate between the indices of the tensor (on the right of the vertical line) and other subscripts (on the left). This notation will be adopted henceforth.

The boundary conditions are reported in (2.23), (2.25) and (2.26). The scaled moment equations are

$$
\begin{gathered}
\sigma_{A}=\int_{-\infty}^{\infty} \phi_{A} E(\boldsymbol{v}) \mathrm{d} \boldsymbol{v}, \\
\bar{v}_{A \mid i}=\int_{-\infty}^{\infty} v_{\mathrm{i}} \phi_{A} E(\boldsymbol{v}) \mathrm{d} \boldsymbol{v}, \\
\frac{3}{2} \tau_{A}=\int_{-\infty}^{\infty}\left(v_{i}^{2}-\frac{3}{2}\right) \phi_{A} E(\boldsymbol{v}) \mathrm{d} \boldsymbol{v}, \\
\frac{\epsilon_{A \mid i j}}{2}=\int_{-\infty}^{\infty} v_{i} v_{j} \phi_{A} E(\boldsymbol{v}) \mathrm{d} \boldsymbol{v},
\end{gathered}
$$

and the pressure is

$$
P_{A}=\sigma_{A}+\tau_{A}
$$

The subscript $A$ in (3.2) and (3.3) takes the values $H, K$ or $C$, i.e. the expressions are equivalent for the Hilbert (bulk flow), Knudsen and collisionless layer quantities. The function $E$ is defined in (2.24). We emphasize that all variables in (3.1)-(3.3) represent their scaled quantities. The perturbation to the mass distribution function $\phi_{A}$, and all moments of this function for the density, mean velocity, temperature and stress tensor of the gas, i.e. $\sigma_{A}, \bar{v}_{A \mid i}, \tau_{A}$ and $\epsilon_{A \mid i j}$, respectively, are the deviations from the equilibrium gas state. Henceforth, this interpretation shall be implicitly assumed, e.g. we refer to $\phi_{A}$ as the mass distribution function, and $\sigma_{A}$ as the density, thus omitting the term 'perturbation'. The full solutions may be constructed from (2.6) and (2.19).

\subsection{Outline of analysis}

For all $A$, which takes the values $H, K$ or $C$, we expand $\phi_{A}$ and all moments of this function in an asymptotic series in the small parameter $\theta^{-1}$, i.e.

$$
\alpha_{A}=\sum_{n=0}^{\infty} \alpha_{A}^{(n)} \theta^{-n},
$$

where $\alpha_{A}$ represents any of these dependent functions, and $\alpha_{A}^{(n)}$ is the $n$ th-order component in the $\theta^{-1}$-expansion. We perform this procedure for all of (i) the linearized Boltzmann-BGK equations in (3.1), (ii) the moment equations in (3.2), 
and (iii) the diffuse reflection, zero net mass flux and decay conditions in (2.23), (2.25) and (2.26). The form of the expansion in (3.4) is identical in all three physically distinct regions, while the equations reported in (3.1) that govern the flows in these regions differ.

Substituting (3.4) into the bulk flow equation in the top line of (3.1) and equating powers of $\theta^{-1}$ yields the required relations for the $n$ th-order terms in the $\theta^{-1}$ expansion of $\phi_{H}$. Evaluating the moments of these expressions using (3.2) produces the relevant hydrodynamic equations at each order in the inverse frequency ratio, $\theta^{-1}$. For completeness, we also evaluate all components of the stress tensor $\epsilon_{i j}$ in the gas, which together with the hydrodynamic equations, are reported up to first order, i.e. $n=1$, in $\S 3.2$.

An equivalent analysis is undertaken for both the Knudsen layer and collisionless layer equations from (3.1). In $\S 3.3$, we prove that the zero solution is the unique solution to the Knudsen layer equation, to infinite order $n$ in the inverse frequency ratio $\theta^{-1}$.

Analysis of the collisionless layer is given in $\$ 3.4$, and yields a collisionless Boltzmann equation in the local wall-normal coordinate $\eta_{C}$ to leading order $(n=0)$. This is solved exactly for the leading-order mass distribution $\phi_{C}^{(0)}$. Explicit equations for the density, mean velocity, temperature and stress tensor of the gas are presented, in terms of the reflected gas particle density, the mean velocity and temperature of the wall, and the applied body force. We remind the reader that the density of reflected gas particles from the wall is chosen to ensure zero net mass flux; see (2.23).

A similar analysis at first order $(n=1)$ also yields explicit solutions for $\phi_{C}^{(1)}$ and its moments, that are valid for a general wall geometry. As before, the equations are expressed in terms of the density of reflected gas particles at the wall, the wall mean velocity and temperature, and the applied body force; additional terms proportional to the normal and geodesic curvatures of the wall also arise. These algebraic formulae are relegated to appendix $\mathrm{A}$, by virtue of their size.

To summarize, we show that flow in the Hilbert region to leading order in $\theta^{-1}$ is inviscid and determined by solution of the linearized Euler equation in $\S 3.2$. Higher-order terms are proportional to gradients in the body force. We prove that the zero solution uniquely satisfies the Knudsen layer equation in $\S 3.3$. Flow within the thin collisionless layer near the wall is free-molecular in the wall-normal direction to leading order; at higher order, advection parallel to the wall and inter-particle collisions also influence the flow. Explicit solutions for the mass distribution function at leading and first order in $\theta^{-1}$ are given in $\$ \$ 3.4 .1$ and 3.4.2, which yield formulae for the hydrodynamic quantities in terms of the mean velocity and temperature of the wall, the density of reflected particles from the wall, and the applied body force.

\subsection{Hilbert expansion and the bulk flow hydrodynamic equations}

Proceeding as outlined above, we arrive at the following expressions for $\phi_{H}^{(n)}$ :

$$
\begin{aligned}
& -\mathrm{i} \phi_{H}^{(0)}=2 a_{i} v_{i}, \\
& -\mathrm{i} \phi_{H}^{(n)}=\sigma_{H}^{(n-1)}-\phi_{H}^{(n-1)}+2 \bar{v}_{H \mid i}^{(n-1)} v_{i}+\left(v_{i}^{2}-\frac{3}{2}\right) \tau_{H}^{(n-1)}-k v_{i} \frac{\partial \phi_{H}^{(n-1)}}{\partial x_{i}}, \quad n \geqslant 1 .
\end{aligned}
$$

Interestingly, all components of the mass distribution function $\phi_{H}^{(n)}$, for all $n \geqslant 0$, are determined explicitly and in complete generality by (3.5). For any given applied body force $a_{i}$, the first line in (3.5) yields the leading-order component, $\phi_{H}^{(0)}$. The 


$$
\begin{array}{lcc}
n=0 & n=1 \\
\phi_{H}^{(0)}=2 \mathrm{i} a_{m} v_{m} & \phi_{H}^{(1)}=2 k v_{m} v_{l} \frac{\partial a_{m}}{\partial x_{l}} \\
\sigma_{H}^{(0)}=0 & \sigma_{H}^{(1)}=k \frac{\partial a_{m}}{\partial x_{m}} \\
P_{H}^{(0)}=0 & P_{H}^{(1)}=\frac{5 k}{3} \frac{\partial a_{m}}{\partial x_{m}} \\
\tau_{H}^{(0)}=0 & \tau_{H}^{(1)}=\frac{2 k}{3} \frac{\partial a_{m}}{\partial x_{m}} \\
\epsilon_{H \mid i j}^{(0)}=0 & \epsilon_{H \mid i j}^{(1)}=k\left(\frac{\partial a_{i}}{\partial x_{j}}+\frac{\partial a_{j}}{\partial x_{i}}+\delta_{i j} \frac{\partial a_{m}}{\partial x_{m}}\right) \\
-\mathrm{i} \bar{v}_{H \mid i}^{(0)}=a_{i} & \bar{v}_{H \mid i}^{(1)}=0
\end{array}
$$

TABLE 1. Outer flow field hydrodynamic relations to first order $(n=1)$ in the inverse frequency ratio $\theta^{-1}$.

higher-order corrections in $\theta^{-1}$ can be found iteratively. The leading-order $(n=0)$ and first-order $(n=1)$ components of the mass distribution function, and all the moments from (3.2), are reported in table 1; the symbol $\delta_{i j}$ is the usual Kronecker delta function.

From table 1, the bulk gas flow in the asymptotic limit $\theta^{-1} \rightarrow 0$ is inviscid; see the hydrodynamic equation for $\bar{v}_{H \mid i}^{(0)}$, which is the linearized Euler equation. Also, the flow at leading order is isobaric; the only forcing term that appears in the Euler equation is the applied body force. In addition, the gas is isothermal at this order, while all components of the stress tensor are zero. For small and finite $\theta^{-1}$, the first-order $(n=1)$ gas density, temperature, and pressure, as well as all components of the stress tensor, are non-zero; see the $n=1$ quantities in table 1 . These all depend on spatial inhomogeneities in the body force. In contrast, the first-order mean velocity is zero. As such, the leading-order solution for the bulk flow mean velocity $\bar{v}_{H \mid i}^{(0)}$ is accurate to $O\left(\theta^{-2}\right)$ away from the walls.

Critically, the equations in table 1 are explicit in terms of the applied body force. No differential equations arise. This contrasts with the low frequency ratio formulation by Nassios \& Sader (2012) and Takata \& Hattori (2012), where a set of integral equations were derived for $\phi_{H}^{(n)}$. In this case, by enforcing conservation of mass, momentum and energy, a coupled set of differential hydrodynamic equations for the moments of $\phi_{H}^{(n)}$ are derived, at each order $n$.

\subsection{Knudsen layer analysis}

Substituting (3.4) into the Knudsen layer equation from (3.1) and equating powers of $\theta^{-1}$ results in the following expressions for $\phi_{K}^{(n)}$ :

$$
\begin{aligned}
-\mathrm{i} \phi_{K}^{(0)}= & 0, \\
-\mathrm{i} \phi_{K}^{(n)}= & \sigma_{K}^{(n-1)}-\phi_{K}^{(n-1)}+2 \bar{v}_{K \mid i}^{(n-1)} v_{i}+\left(v_{i}^{2}-\frac{3}{2}\right) \tau_{K}^{(n-1)}-v_{i} n_{i} \frac{\partial \phi_{K}^{(n-1)}}{\partial \eta_{K}} \\
& -k\left(v_{i} t_{i}^{1} t_{j}^{1} \frac{\partial \phi_{K}^{(n-1)}}{\partial x_{j}}+v_{i} t_{i}^{2} t_{j}^{2} \frac{\partial \phi_{K}^{(n-1)}}{\partial x_{i}}\right), \quad n \geqslant 1 .
\end{aligned}
$$


It is clear from the first line of (3.6) that

$$
\phi_{K}^{(0)}=0 .
$$

In appendix $\mathrm{B}$, we use (3.7) to prove that $\phi_{K}^{(n)} \equiv 0$ for all $n \geqslant 0$ in the Knudsen layer using the method of induction. The problem thus reduces to a classical matched asymptotic expansion between two unique solutions: the bulk flow represented by a subscript $H$, and the collisionless layer represented by a subscript $C$. An analysis of the collisionless layer is the focus of the following subsection.

\subsection{Collisionless layer analysis}

We now substitute (3.4) into the collisionless layer equation in (3.1). Equating powers of $\theta^{-1}$, we arrive at a set of equations for $\phi_{C}^{(n)}$ of general form,

$$
-\mathrm{i} \phi_{C}^{(n)}+v_{i} n_{i} \frac{\partial \phi_{C}^{(n)}}{\partial \eta_{C}}=G^{(n)}\left(\eta_{C}\right)+K^{(n)}\left(\eta_{C}\right)
$$

where $G^{(n)}\left(\eta_{C}\right)$ and $K^{(n)}\left(\eta_{C}\right)$ are defined as

$$
\begin{gathered}
G^{(n)}\left(\eta_{C}\right)= \begin{cases}0, & n=0, \\
\sigma_{C}^{(n-1)}-\phi_{C}^{(n-1)}+2 \bar{v}_{C \mid i}^{(n-1)} v_{i}+\left(v_{i}^{2}-\frac{3}{2}\right) \tau_{C}^{(n-1)}, & n \geqslant 1,\end{cases} \\
K^{(n)}\left(\eta_{C}\right)= \begin{cases}0, & n=0, \\
-k\left(v_{j} t_{j}^{1} t_{i}^{1} \frac{\partial \phi_{C}^{(n-1)}}{\partial x_{i}}+v_{j} t_{j}^{2} t_{i}^{2} \frac{\partial \phi_{C}^{(n-1)}}{\partial x_{i}}\right), & n \geqslant 1 .\end{cases}
\end{gathered}
$$

In (3.9) and (3.10), all collisionless layer quantities are presented with no argument for simplicity of notation, e.g. $\sigma_{C}^{(n-1)}$ implicitly takes the argument $\eta_{C}$. Setting $n=0$ in (3.8)-(3.10) yields a collisionless Boltzmann equation for $\phi_{C}^{(0)}$ in the spatial coordinate $\eta_{C}$; this is the scaled wall-normal coordinate introduced in $\S 2.1$. For a slightly rarefied gas, the leading-order $(n=0)$ flow within the collisionless layer corresponds to freemolecular flow of reflected gas particles away from the wall, i.e. in the wall-normal direction only.

The differential equations that specify the higher-order $(n \geqslant 1)$ components of the mass distribution function $\phi_{C}^{(n)}$ are of similar form; see (3.8). However, inhomogeneous terms are clearly present on the right-hand side of (3.8). For small and finite $\theta^{-1}$, these terms are non-zero in general and alter the flow; see (3.9) and (3.10). To facilitate a clear physical description, we write the inhomogeneities at $O\left(\theta^{-n}\right)$ as the sum of two components, $G^{(n)}$ and $K^{(n)}$. For $n \geqslant 1, G^{(n)}$ is functionally identical to the linearized BGK approximation; see (3.9) and the right-hand side of the first line of (3.1). This term accounts for the influence of inter-particle collisions on the flow at small and finite $\theta^{-1}$. The second component, $K^{(n)}$, is proportional to the scaled Knudsen number $k$ and accounts for advective transport of gas particles parallel to the wall; this is clear from (3.10). For an arbitrary wall shape, we analyse this term using generalized coordinates and the method of moving frame from $\S 2.2$.

As we shall show, the mass distribution function in the collisionless layer, $\phi_{C}^{(n)}$, is a discontinuous function of the wall-normal particle velocity, $v_{i} n_{i}$, at all orders $n$. This is a result of the presence of the wall, and the contrasting conditions applied to particles incident upon the wall and those reflected from the wall. Importantly, both $G^{(n+1)}$ and 
$K^{(n+1)}$ are functions of $\phi_{C}^{(n)}$, for $n \geqslant 0$. Their respective functional forms will thus differ for incident particles $\left(v_{i} n_{i}<V_{i} n_{i}\right)$ and reflected particles $\left(v_{i} n_{i} \geqslant V_{i} n_{i}\right)$.

Next, we present the solutions to (3.8) up to and including all first-order $(n=1)$ terms in the inverse frequency ratio $\theta^{-1}$. We impose the diffuse reflection boundary condition with zero net mass flux at the wall position $\left(\eta_{C}=0\right)$, and require all collisionless layer quantities to decay rapidly for increasing $\eta_{C}$; see (2.23), (2.25) and (2.26). The analysis of the leading-order equation $(n=0)$ is presented in $\S 3.4 .1$. The first-order analysis is given in $\$ 3.4 .2$; the equations for the hydrodynamic quantities at this order are extensive, and relegated to appendix A.

\subsubsection{Leading-order analysis $(n=0)$}

We define the leading-order mass distribution function for incident particles as $\phi_{C \mid v_{i} n_{i}<V_{i} n_{i}}^{(0)}$, and the corresponding quantity for reflected particles as $\phi_{C \mid v_{i} n_{i} \geqslant V_{i} n_{i}}^{(0)}$. Setting $n=0$ in (3.8) and substituting for $G^{(0)}$ and $K^{(0)}$ from (3.9) and (3.10), the resulting differential equations for both $\phi_{C \mid v_{i} n_{i}<V_{i} n_{i}}^{(0)}$ and $\phi_{C \mid v_{i} n_{i} \geqslant V_{i} n_{i}}^{(0)}$ are identical. This is because $G^{(0)}$ and $K^{(0)}$ are both uniquely zero; see (3.9) and (3.10). As discussed in $\S 2.3$, the collisionless layer is localized near the wall. For large $\eta_{C}$, we therefore require that $\phi_{C \mid v_{i} n_{i}<V_{i} n_{i}}^{(0)}$ decay in accordance with (2.26), which gives

$$
\begin{gathered}
-\mathrm{i} \phi_{C \mid v_{i} n_{i}<V_{i} n_{i}}^{(0)}+v_{i} n_{i} \frac{\partial \phi_{C \mid v_{i} n_{i}<V_{i} n_{i}}^{(0)}=0}{\partial \eta_{C}}=0 \\
0=\lim _{\eta_{C} \rightarrow \infty} \phi_{C \mid v_{i} n_{i}<V_{i} n_{i}}^{(0)} .
\end{gathered}
$$

Since particles undergo diffuse reflection with zero net mass flux from the wall, $\phi_{C \mid v_{i} n_{i} \geqslant V_{i} n_{i}}^{(0)}$ must satisfy

$$
\begin{gathered}
-\mathrm{i} \phi_{C \mid v_{i} n_{i} \geqslant V_{i} n_{i}}^{(0)}+v_{i} n_{i} \frac{\partial \phi_{C \mid v_{i} n_{i} \geqslant V_{i} n_{i}}^{(0)}}{\partial \eta_{C}}=0, \\
\left.\phi_{C \mid v_{i} n_{i} \geqslant V_{i} n_{i}}\right|_{\eta_{C}=0}=\sigma_{b}^{(0)}+2 V_{i}^{(0)} v_{i}+\left(v_{i}^{2}-\frac{3}{2}\right) \tau_{b}^{(0)}-\left.\phi_{H}^{(0)}\right|_{\eta_{C}=0},
\end{gathered}
$$

where the reflected particle density is given by

$$
\begin{aligned}
\sigma_{b}^{(0)}= & \sqrt{\pi} V_{i}^{(0)} n_{i}-\frac{1}{2} \tau_{b}^{(0)} \\
& -\left.2 \sqrt{\pi} \int_{-\infty}^{\infty} \int_{-\infty}^{\infty} \int_{-\infty}^{0} \xi \phi_{C \mid \xi \geqslant 0}^{(0)}\right|_{\eta_{C}=0} E\left(v_{i} t_{i}^{1}, v_{i} t_{i}^{2}, \xi\right) \mathrm{d} \xi \mathrm{d}\left(v_{i} t_{i}^{1}\right) \mathrm{d}\left(v_{i} t_{i}^{2}\right) .
\end{aligned}
$$

The integration variable $\xi$ is defined in (2.23). The solution of (3.11)-(3.13) yields the required result for $\phi_{C}^{(0)}$, for all $v_{i} n_{i}$,

$$
\phi_{C}^{(0)}= \begin{cases}\left(P_{b}^{(0)}+2 c_{i}^{(0)} v_{i}+\left(v_{i}^{2}-\frac{5}{2}\right) \tau_{b}^{(0)}\right) \exp \left(\frac{\mathrm{i} \eta_{c}}{v_{i} n_{i}}\right), & v_{i} n_{i} \geqslant V_{i} n_{i}, \\ 0, & v_{i} n_{i}<V_{i} n_{i},\end{cases}
$$

where $P_{b}^{(0)}=\sigma_{b}^{(0)}+\tau_{b}^{(0)}$ and

$$
c_{i}^{(0)}=V_{i}^{(0)}-\left.\mathrm{i} a_{i}\right|_{\eta_{c}=0}
$$




$$
\begin{array}{lc} 
& P_{b}^{(0)} J_{0}\left(-\mathrm{i} \eta_{C}\right)+2 c_{i}^{(0)} n_{i} J_{1}\left(-\mathrm{i} \eta_{C}\right) \\
\sqrt{\pi} \sigma_{C}^{(0)} & +\tau_{b}^{(0)}\left(J_{2}\left(-\mathrm{i} \eta_{C}\right)-\frac{3}{2} J_{0}\left(-\mathrm{i} \eta_{C}\right)\right) \\
& P_{b}^{(0)} J_{1}\left(-\mathrm{i} \eta_{C}\right)+2 c_{i}^{(0)} n_{i} J_{2}\left(-\mathrm{i} \eta_{C}\right) \\
\sqrt{\pi} \bar{v}_{C \mid i}^{(0)} n_{i} & +\tau_{b}^{(0)}\left(J_{3}\left(-\mathrm{i} \eta_{C}\right)-\frac{3}{2} J_{1}\left(-\mathrm{i} \eta_{C}\right)\right) \\
\sqrt{\pi} \bar{v}_{C \mid i} t_{i}^{1,2} & c_{i}^{(0)} t_{i}^{1,2} J_{0}\left(-\mathrm{i} \eta_{C}\right) \\
& P_{b}^{(0)}\left(J_{2}\left(-\mathrm{i} \eta_{C}\right)-\frac{1}{2} J_{0}\left(-\mathrm{i} \eta_{C}\right)\right) \\
\frac{3}{2} \sqrt{\pi} \tau_{C}^{(0)} & +2 c_{i}^{(0)} n_{i}\left(J_{3}\left(-\mathrm{i} \eta_{C}\right)-\frac{1}{2} J_{1}\left(-\mathrm{i} \eta_{C}\right)\right) \\
\frac{\sqrt{\pi}}{2} \epsilon_{C \mid i j}^{(0)} n_{i} n_{j} & +\tau_{b}^{(0)}\left(J_{4}\left(-\mathrm{i} \eta_{C}\right)-2 J_{2}\left(-\mathrm{i} \eta_{C}\right)+\frac{7}{4} J_{0}\left(-\mathrm{i} \eta_{C}\right)\right) \\
\frac{\sqrt{\pi}}{2} \epsilon_{C \mid i j}^{(0)} n_{i} t_{j}^{1,2}=\frac{\sqrt{\pi}}{2} \epsilon_{C \mid j i}^{(0)} n_{i} t_{j}^{1,2} & P_{b}^{(0)} J_{2}\left(-\mathrm{i} \eta_{C}\right)+2 c_{i}^{(0)} n_{i} J_{3}\left(-\mathrm{i} \eta_{C}\right) \\
\epsilon_{C \mid j i}^{(0)} t_{i}^{1,2} t_{j}^{2,1}=\epsilon_{C \mid j i}^{(0)} t_{i}^{1,2} t_{j}^{2,1} & +\tau_{b}^{(0)}\left(J_{4}\left(-\mathrm{i} \eta_{C}\right)-\frac{3}{2} J_{2}\left(-\mathrm{i} \eta_{C}\right)\right) \\
\sqrt{\pi} \epsilon_{C \mid i j}^{(0)} t_{i}^{1} t_{j}^{1}=\sqrt{\pi} \epsilon_{C \mid i j}^{(0)} t_{i}^{2} t_{j}^{2} & P_{b}^{(0)} J_{0}\left(-\mathrm{i} \eta_{C}\right)+2 c_{i}^{(0)} n_{i} J_{1}\left(-\mathrm{i} \eta_{C}\right) \\
& +\tau_{b}^{(0)}\left(J_{2}\left(-\mathrm{i} \eta_{C}\right)-\frac{1}{2} J_{0}\left(-\mathrm{i} \eta_{C}\right)\right) \\
& 0
\end{array}
$$

TABLE 2. The macroscopic flow quantities and stress tensor components to leading order $(n=0)$ in the inverse frequency ratio $\theta^{-1}$ within the collisionless layer.

The solution in (3.14) is a free-molecular solution, where advection of reflected gas particles occurs in the normal direction from the wall only.

To summarize, using the applied body force $a_{i}$, and the mean velocity $V_{i}^{(0)}$ and temperature $\tau_{b}^{(0)}$ of the wall, the density of particles reflected from the wall $\sigma_{b}^{(0)}$ may be evaluated using (3.13). Equation (3.14) is therefore an algebraic expression for $\phi_{C}^{(0)}$, which is the leading-order $(n=0)$ mass distribution function within the collisionless layer.

Substituting (3.14) into the moment relations in (3.2) also yields algebraic expressions for the hydrodynamic quantities within the collisionless layer, at leading order in $\theta^{-1}$. The density, mean velocity and temperature of the gas, and all components of the gas stress tensor, are therefore determined explicitly and in complete generality at this order; see table 2, where the Abramowitz functions $J_{n}(\eta)$ are defined by (Abramowitz \& Stegun 1965)

$$
J_{n}(\eta)=\int_{0}^{\infty} t^{n} \exp \left(-\frac{\eta}{t}-t^{2}\right) \mathrm{d} t .
$$




$$
\begin{array}{lc}
\epsilon_{C \mid i j}^{(0)} n_{i} n_{j} & \frac{1}{2} P_{b}^{(0)}+\frac{2}{\sqrt{\pi}} c_{i}^{(0)} n_{i} \\
\epsilon_{C \mid i j}^{(0)} t_{i}^{1} t_{j}^{1}=\epsilon_{C|j| j}^{(0)} t_{i}^{2} t_{j}^{2} & \frac{1}{2} P_{b}^{(0)}+\frac{1}{\sqrt{\pi}} c_{i}^{(0)} n_{i} \\
\epsilon_{C \mid i j}^{(0)} n_{i} t_{j}^{1,2}=\epsilon_{C \mid j}^{(0)} n_{i} t_{j}^{1,2} & \frac{1}{\sqrt{\pi}} c_{i}^{(0)} t_{i}^{1,2} \\
\epsilon_{C \mid i j}^{(0)} t_{i}^{1,2} t_{j}^{2,1}=\epsilon_{C \mid i j}^{(0)} t_{i}^{1,2} t_{j}^{2,1} & 0
\end{array}
$$

TABLE 3. The leading-order stress tensor components $\epsilon_{i j}^{(0)}$ in the collisionless layer evaluated at the solid wall, $\eta_{C}=0$. The solutions are evaluated using the generalized representation of the Abramowitz functions in $\S \mathrm{C}$.

The Abramowitz functions are discussed further in appendix C. At $\eta=0$, the $J_{n}$ functions reduce to Gaussian moment integrals; see (3.16). This property is exploited to provide algebraic expressions for each component of the leading-order stress tensor $\epsilon_{i j}^{(0)}$ at the wall $\eta_{C}=0$, in terms of $a_{i}, V_{i}^{(0)}, \tau_{b}^{(0)}$, and $P_{b}^{(0)}=\sigma_{b}^{(0)}+\tau_{b}^{(0)}$; see table 3 .

\subsubsection{First-order analysis $(n=1)$}

We now turn our attention to solution of the governing equation in (3.8) at next order, i.e. $n=1$. Setting $n=1$ in (3.8)-(3.10) yields two distinct, inhomogeneous first-order differential equations for $\phi_{C \mid v_{i} n_{i}<V_{i} n_{i}}^{(1)}$ and $\phi_{C \mid v_{i} n_{i} \geqslant V_{i} n_{i}}^{(1)}$. The differential equation and boundary condition for the mass distribution function of incident particles is

$$
\begin{aligned}
& \frac{\partial \phi_{C \mid v_{i} n_{i}<V_{i} n_{i}}^{(1)}}{\partial y}-\frac{\mathrm{i}}{v_{i} n_{i}} \phi_{C \mid v_{i} n_{i}<V_{i} n_{i}}^{(1)}=\frac{1}{v_{i} n_{i}}\left(G_{v_{i} n_{i}<V_{i} n_{i}}^{(1)}\left(\eta_{C}\right)+K_{v_{i} n_{i}<V_{i} n_{i}}^{(1)}\left(\eta_{C}\right)\right), \\
& 0=\lim _{\eta_{C} \rightarrow \infty} \phi_{C \mid v_{i} n_{i}<V_{i} n_{i}}^{(1)} .
\end{aligned}
$$

The corresponding equation for particles reflected from the wall is

$$
\begin{gathered}
\frac{\partial \phi_{C \mid v_{i} n_{i} \geqslant V_{i} n_{i}}^{(1)}-\frac{\mathrm{i}}{\partial \eta_{C}} \phi_{i} n_{i}}{(1)}=\frac{1}{v_{i} n_{i} \geqslant V_{i} n_{i}}\left(G_{v_{i} n_{i} \geqslant V_{i} n_{i}}^{(1)}\left(\eta_{C}\right)+K_{v_{i} n_{i} \geqslant V_{i} n_{i}}^{(1)}\left(\eta_{C}\right)\right), \\
\left.\phi_{C \mid v_{i} n_{i} \geqslant V_{i} n_{i}}^{(1)}\right|_{\eta_{C}=0}=\sigma_{b}^{(1)}+2 V_{i}^{(1)} v_{i}+\left(v_{i}^{2}-\frac{3}{2}\right) \tau_{b}^{(1)}-\left.\phi_{H}^{(1)}\right|_{\eta_{C}=0},
\end{gathered}
$$

where the reflected particle density is

$$
\begin{aligned}
\sigma_{b}^{(1)}= & \sqrt{\pi} V_{i}^{(1)} n_{i}-\frac{1}{2} \tau_{b}^{(1)} \\
& -\left.2 \sqrt{\pi} \int_{-\infty}^{\infty} \int_{-\infty}^{\infty} \int_{-\infty}^{0} \xi \phi_{C \mid \xi \geqslant 0}^{(1)}\right|_{\eta_{C}=0} E\left(v_{i} t_{i}^{1}, v_{i} t_{i}^{2}, \xi\right) \mathrm{d} \xi \mathrm{d}\left(v_{i} t_{i}^{1}\right) \mathrm{d}\left(v_{i} t_{i}^{2}\right) .
\end{aligned}
$$

As discussed earlier, $G^{(1)}$ and $K^{(1)}$ are functions of $\phi_{C}^{(0)}$, which is discontinuous in $v_{i} n_{i}$. Substituting the results in table 2 and (3.14), into (3.9), we find that $G^{(1)}$ for incident 
particles takes the form

$$
\begin{aligned}
G_{v_{i} n_{i}<V_{i} n_{i}}^{(1)}\left(\eta_{C}\right)= & \frac{1}{\sqrt{\pi}}\left[P _ { b } ^ { ( 0 ) } \left(J_{0}\left(-\mathrm{i} \eta_{C}\right)+2 v_{i} n_{i} J_{1}\left(-\mathrm{i} \eta_{C}\right)+\frac{2}{3}\left(v_{i}^{2}-\frac{3}{2}\right)\left(J_{2}\left(-\mathrm{i} \eta_{C}\right)\right.\right.\right. \\
& \left.\left.-\frac{1}{2} J_{0}\left(-\mathrm{i} \eta_{C}\right)\right)\right)+2 c_{i}^{(0)} n_{i}\left(J_{1}\left(-\mathrm{i} \eta_{C}\right)\right. \\
& \left.+2 v_{i} n_{i} J_{2}\left(-\mathrm{i} \eta_{C}\right)+\frac{2}{3}\left(v_{i}^{2}-\frac{3}{2}\right)\left(J_{3}\left(-\mathrm{i} \eta_{C}\right)-\frac{1}{2} J_{1}\left(-\mathrm{i} \eta_{C}\right)\right)\right) \\
& +2 J_{0}\left(-\mathrm{i} \eta_{C}\right)\left(v_{j} t_{j}^{1} c_{i}^{(0)} t_{i}^{1}+v_{j} t_{j}^{2} c_{i}^{(0)} t_{i}^{2}\right)+\tau_{b}^{(0)}\left(J_{2}\left(-\mathrm{i} \eta_{C}\right)-\frac{3}{2} J_{0}\left(-\mathrm{i} \eta_{C}\right)\right. \\
& +2 v_{i} n_{i}\left(J_{3}\left(-\mathrm{i} \eta_{C}\right)-\frac{3}{2} J_{1}\left(-\mathrm{i} \eta_{C}\right)\right)+\frac{2}{3}\left(v_{i}^{2}-\frac{3}{2}\right)\left(J_{4}\left(-\mathrm{i} \eta_{C}\right)\right. \\
& \left.\left.\left.-2 J_{2}\left(-\mathrm{i} \eta_{C}\right)+\frac{7}{4} J_{0}\left(-\mathrm{i} \eta_{C}\right)\right)\right)\right]
\end{aligned}
$$

while the corresponding expression for reflected particles is

$$
\begin{aligned}
G_{v_{i} n_{i} \geqslant V_{i} n_{i}}^{(1)}\left(\eta_{C}\right)= & \frac{1}{\sqrt{\pi}}\left[P _ { b } ^ { ( 0 ) } \left(J_{0}\left(-\mathrm{i} \eta_{C}\right)-\sqrt{\pi} \exp \left(\frac{\mathrm{i} \eta_{C}}{v_{i} n_{i}}\right)+2 v_{i} n_{i} J_{1}\left(-\mathrm{i} \eta_{C}\right)\right.\right. \\
& \left.+\frac{2}{3}\left(v_{i}^{2}-\frac{3}{2}\right)\left(J_{2}\left(-\mathrm{i} \eta_{C}\right)-\frac{1}{2} J_{0}\left(-\mathrm{i} \eta_{C}\right)\right)\right)+2 c_{i}^{(0)} n_{i} \\
& \times\left(J_{1}\left(-\mathrm{i} \eta_{C}\right)-\sqrt{\pi} v_{i} n_{i} \exp \left(\frac{\mathrm{i} \eta_{C}}{v_{i} n_{i}}\right)+2 v_{i} n_{i} J_{2}\left(-\mathrm{i} \eta_{C}\right)\right. \\
& \left.+\frac{2}{3}\left(v_{i}^{2}-\frac{3}{2}\right)\left(J_{3}\left(-\mathrm{i} \eta_{C}\right)-\frac{1}{2} J_{1}\left(-\mathrm{i} \eta_{C}\right)\right)\right)+2\left(v_{j} t_{j}^{1} c_{i}^{(0)} t_{i}^{1}\right. \\
& \left.+v_{j} t_{j}^{2} c_{i}^{(0)} t_{i}^{2}\right)\left(J_{0}\left(-\mathrm{i} \eta_{C}\right)-\sqrt{\pi} \exp \left(\frac{\mathrm{i} \eta_{C}}{v_{i} n_{i}}\right)\right) \\
& +\tau_{b}^{(0)}\left(J_{2}\left(-\mathrm{i} \eta_{C}\right)-\frac{3}{2} J_{0}\left(-\mathrm{i} \eta_{C}\right)+2 v_{i} n_{i}\left(J_{3}\left(-\mathrm{i} \eta_{C}\right)\right.\right. \\
& \left.-\frac{3}{2} J_{1}\left(-\mathrm{i} \eta_{C}\right)\right)-\sqrt{\pi}\left(v_{i}^{2}-\frac{5}{2}\right) \exp \left(\frac{\mathrm{i} \eta_{C}}{v_{i} n_{i}}\right) \\
& \left.\left.+\frac{2}{3}\left(v_{i}^{2}-\frac{3}{2}\right)\left(J_{4}\left(-\mathrm{i} \eta_{C}\right)-2 J_{2}\left(-\mathrm{i} \eta_{C}\right)+\frac{7}{4} J_{0}\left(-\mathrm{i} \eta_{C}\right)\right)\right)\right]
\end{aligned}
$$

Substituting (3.14) into (3.10), we find that $K^{(1)}$ for incident particles is uniquely zero:

$$
K_{v_{i} n_{i}<V_{i} n_{i}}^{(1)}\left(\eta_{C}\right)=0 .
$$

Using the results in $\S 2.2$, the expression for reflected gas particles is non-zero and is given by

$$
\begin{aligned}
K_{v_{i} n_{i} \geqslant V_{i} n_{i}}^{(1)}\left(\eta_{C}\right)= & -k \exp \left(\frac{\mathrm{i} \eta_{C}}{v_{i} n_{i}}\right)\left[\left(v_{j} t_{j}^{1} t_{i}^{1} \frac{\partial}{\partial x_{i}}+v_{j} t_{j}^{2} t_{i}^{2} \frac{\partial}{\partial x_{i}}\right)\left(P_{b}^{(0)}+\left[v_{i}^{2}-\frac{5}{2}\right] \tau_{b}^{(0)}\right)\right. \\
& -\frac{\mathrm{i} \eta_{C}}{\left(v_{i} n_{i}\right)^{2}} v_{i} \kappa_{i j} v_{j}\left(P_{b}^{(0)}+2 c_{i}^{(0)} v_{i}+\left(v_{i}^{2}-\frac{5}{2}\right) \tau_{b}^{(0)}\right)
\end{aligned}
$$




$$
\begin{aligned}
& +2 c_{j}^{(0)} t_{j}^{1}\left(-\kappa_{1} v_{s} t_{s}^{1} v_{i} n_{i}+g_{1} v_{s} t_{s}^{1} v_{i} t_{i}^{2}+g_{2}\left[v_{i} t_{i}^{2}\right]^{2}\right) \\
& -2 c_{j}^{(0)} t_{j}^{2}\left(\kappa_{2} v_{s} t_{s}^{2} v_{i} n_{i}+g_{1}\left[v_{i} t_{i}^{1}\right]^{2}+g_{2} v_{s} t_{s}^{1} v_{i} t_{i}^{2}\right) \\
& +2 c_{j}^{(0)} n_{j} v_{i} \kappa_{i j} v_{j}+2 v_{j} t_{j}^{1}\left(v_{j} t_{j}^{1} t_{i}^{1} \frac{\partial}{\partial x_{i}}+v_{j} t_{j}^{2} t_{i}^{2} \frac{\partial}{\partial x_{i}}\right)\left(c_{i}^{(0)} t_{i}^{1}\right) \\
& +2 v_{j} t_{j}^{2}\left(v_{j} t_{j}^{1} t_{i}^{1} \frac{\partial}{\partial x_{i}}+v_{j} t_{j}^{2} t_{i}^{2} \frac{\partial}{\partial x_{i}}\right)\left(c_{i}^{(0)} t_{i}^{2}\right) \\
& \left.+2 v_{j} n_{j}\left(v_{j} t_{j}^{1} t_{i}^{1} \frac{\partial}{\partial x_{i}}+v_{j} t_{j}^{2} t_{i}^{2} \frac{\partial}{\partial x_{i}}\right)\left(c_{i}^{(0)} n_{i}\right)\right] .
\end{aligned}
$$

By linearity and (3.20)-(3.23), the first-order $(n=1)$ correction to the mass distribution function can be written in the general form

$$
\phi_{C}^{(1)}=\phi_{C, f m}^{(1)}+\phi_{C, c o l}^{(1)}+\phi_{C, t a n}^{(1)} .
$$

The three components of $\phi_{C}^{(1)}$ have the algebraic representations

$$
\begin{aligned}
& \phi_{C, f m}^{(1)}= \begin{cases}{\left[\begin{array}{ll}
\sigma_{b}^{(1)}+2 V_{i}^{(1)} v_{i}+\left(v_{i}^{2}-\frac{3}{2}\right) \tau_{b}^{(1)} & \\
\left.-\left.2 k v_{i} v_{j} \frac{\partial a_{j}}{\partial x_{i}}\right|_{\eta_{C}=0}\right] \exp \left(\mathrm{i} \frac{\eta_{C}}{v_{i} n_{i}}\right), & \\
0, & v_{i} n_{i} \geqslant V_{i} n_{i},
\end{array}, V_{i} n_{i},\right.}\end{cases}
\end{aligned}
$$

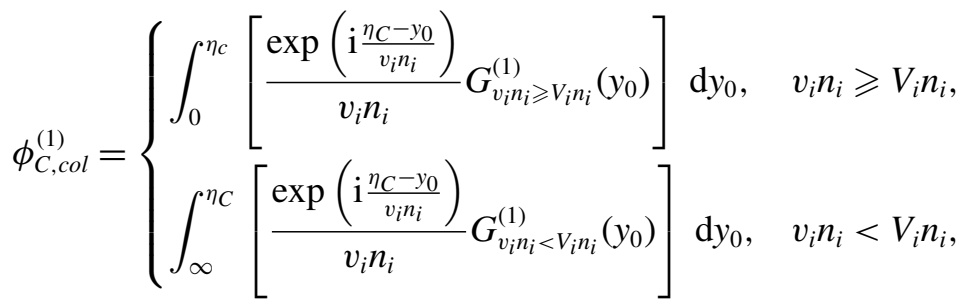

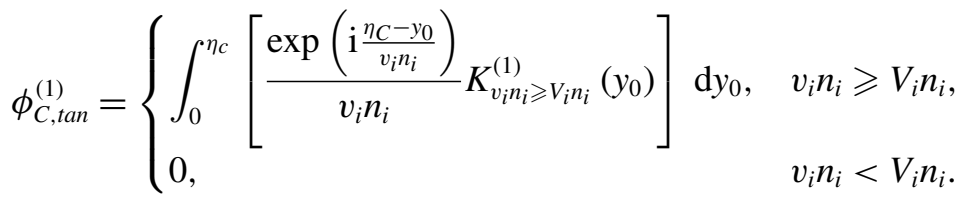

In (3.27), we have made use of (3.22) to eliminate $K_{v_{i} n_{i}<V_{i} n_{i}}^{(1)}$. Mathematically, $\phi_{C, f m}^{(1)}$ is the homogeneous solution to (3.17) and (3.18) for $\phi_{C}^{(1)}$. Physically, this component of $\phi_{C}^{(1)}$ accounts for free-molecular flow of reflected gas particles away from the wall, i.e. in the wall-normal direction only; we remind the reader that this was the only physical mechanism present at leading order $(n=0)$, as discussed in $\$ 3.4 .1$. The second component $\phi_{C, c o l}^{(1)}$ is one part of the particular solution to (3.17) and (3.18); given the dependence upon $G^{(1)}$, this models the effect of inter-particle collisions on the flow, for small but finite inverse frequency ratio $\theta^{-1}$. The third component $\phi_{C \text {,tan }}^{(1)}$ is defined in terms of $K^{(1)}$. It therefore accounts for advection of gas particles parallel to the wall, and the influence of this motion on the flow within the collisionless layer.

Interestingly, (3.24)-(3.27) define a set of algebraic expressions for the first-order $(n=1)$ mass distribution function. As was the case at leading order $(n=0)$, the 


$$
\begin{aligned}
& P_{b}^{(0)}\left(6.33 \times 10^{-7}-0.0700 \mathrm{i}\right)+c_{i}^{(0)} n_{i}\left(2.40 \times 10^{-6}-0.1420 \mathrm{i}\right) \\
& \epsilon_{C \mid i j}^{(1)} n_{i} n_{j} \\
& +\tau_{b}^{(0)}\left(1.54 \times 10^{-6}-0.0582 \mathrm{i}\right)+\frac{P_{b}^{(1)}}{4}+\frac{V_{i}^{(1)} n_{i}}{\sqrt{\pi}} \\
& -\left.\frac{k}{2} \frac{\partial a_{j}}{\partial x_{k}}\right|_{\eta_{C}=0}\left(n_{j} n_{k}+\frac{\delta_{j k}}{2}\right) \\
& P_{b}^{(0)}\left(1.26 \times 10^{-5}-0.0606 \mathrm{i}\right)+c_{i}^{(0)} n_{i}\left(4.23 \times 10^{-6}+0.0853 \mathrm{i}\right) \\
& +\tau_{b}^{(0)}\left(1.62 \times 10^{-5}+0.0237 \mathrm{i}\right)+\frac{P_{b}^{(1)}}{2}+\frac{V_{i}^{(1)} n_{i}}{\sqrt{\pi}} \\
& \epsilon_{C \mid i j}^{(1)} t_{i}^{1} t_{j}^{1} \\
& -\left.\frac{k}{4} \frac{\partial a_{i}}{\partial x_{i}}\right|_{\eta_{C}=0}-\frac{k}{2}\left(t_{k}^{1} \frac{\left.\partial\left(a_{j} t_{j}^{1}\right)\right|_{\eta_{C}=0}}{\partial x_{k}}+\left.\kappa_{1}\left(a_{j} n_{j}\right)\right|_{\eta_{C}=0}\right. \\
& \left.-\left.g_{1}\left(a_{j} t_{j}^{2}\right)\right|_{\eta_{C}=0}\right)+\frac{\mathrm{i} k}{4 \sqrt{\pi}}\left(3 \kappa_{1}+\kappa_{2}\right)\left[P_{b}^{(0)}-\frac{1}{2} \tau_{b}^{(0)}\right] \\
& P_{b}^{(0)}\left(1.26 \times 10^{-5}-0.0606 \mathrm{i}\right)+c_{i}^{(0)} n_{i}\left(4.23 \times 10^{-6}+0.0853 \mathrm{i}\right) \\
& +\tau_{b}^{(0)}\left(1.62 \times 10^{-5}+0.0237 \mathrm{i}\right)+\frac{P_{b}^{(1)}}{2}+\frac{V_{i}^{(1)} n_{i}}{\sqrt{\pi}} \\
& \epsilon_{C \mid i j}^{(1)} t_{i}^{2} t_{j}^{2} \\
& -\left.\frac{k}{4} \frac{\partial a_{i}}{\partial x_{i}}\right|_{\eta_{C}=0}-\frac{k}{2}\left(t_{k}^{2} \frac{\left.\partial\left(a_{j} t_{j}^{2}\right)\right|_{\eta_{C}=0}}{\partial x_{k}}+\left.\kappa_{2}\left(a_{j} n_{j}\right)\right|_{\eta_{C}=0}\right. \\
& \left.+\left.g_{2}\left(a_{j} t_{j}^{1}\right)\right|_{\eta_{C}=0}\right)+\frac{\mathrm{i} k}{4 \sqrt{\pi}}\left(\kappa_{1}+3 \kappa_{2}\right)\left[P_{b}^{(0)}-\frac{1}{2} \tau_{b}^{(0)}\right] \\
& c_{i}^{(0)} t_{i}^{1,2}\left(-2.56 \times 10^{-9}-0.1068 \mathrm{i}\right)+\frac{V_{i} t_{i}^{1,2}}{\sqrt{\pi}} \\
& \epsilon_{C \mid i j}^{(1)} n_{i} t_{j}^{1,2}=\epsilon_{C \mid j i}^{(1)} n_{i} t_{j}^{1,2} \\
& -\left.\frac{k}{4}\left(t_{j}^{1,2} n_{k}+n_{j} t_{k}^{1,2}\right) \frac{\partial a_{j}}{\partial x_{k}}\right|_{\eta_{C}=0} \\
& \epsilon_{C \mid i j}^{(1)} t_{i}^{1,2} t_{j}^{2,1}=\epsilon_{C \mid j i}^{(1)} t_{i}^{1,2} t_{j}^{2,1} \quad-\left.\frac{k}{4}\left(t_{j}^{1,2} t_{k}^{2,1}+t_{j}^{2,1} t_{k}^{1,2}\right) \frac{\partial a_{j}}{\partial x_{k}}\right|_{\eta_{C}=0}
\end{aligned}
$$

TABLE 4. The leading-order stress tensor components $\epsilon_{i j}^{(1)}$ in the collisionless layer evaluated at the solid wall, $\eta_{C}=0$.

resulting expressions are explicit in terms of $a_{i}, P_{b}^{(0)}, V_{i}^{(0)}$ and $\tau_{b}^{(0)}$. Additional terms also appear, which are proportional to the normal and geodesic curvatures of the wall, as well as $P_{b}^{(1)}=\sigma_{b}^{(1)}+\tau_{b}^{(1)}, V_{i}^{(1)}$ and $\tau_{b}^{(1)}$. The moments of $\phi_{C}^{(1)}$ can be evaluated by substituting (3.24) into the moment relations in (3.2). Interchanging the order of integration over particle velocity, $\boldsymbol{v}$, and coordinate space, $y_{0}$, we arrive at an explicit set of expressions for the macroscopic flow quantities, and the gas stress tensor components. These are summarized in (A 2)-(A 12) in appendix A.

For completeness, the first-order $(n=1)$ components of the gas stress tensor are evaluated at the wall $\eta_{C}=0$, with the results reported in table 4 . The figures reported there were calculated using NIntegrate in MATHEMATICA ${ }^{\circledR}$; see the discussion in appendix C. This approach yields solutions for all non-dimensional hydrodynamic 
quantities, including all stress tensor components, which possess a maximum error magnitude of $10^{-4}$ at the wall, i.e. at $\eta_{C}=0$.

Together with the hydrodynamic equations in table 1 and the leading-order corrections due to the collisionless layer in table 2, the set of equations in appendix A gives the gas density, mean velocity, and temperature, and all components of the stress tensor, up to first order in the inverse frequency ratio $\theta^{-1}$. All equations are explicitly specified in terms of $a_{i}$, and $P_{b}^{(n)}=\sigma_{b}^{(n)}+\tau_{b}^{(n)}, V_{i}^{(n)}$ and $\tau_{b}^{(n)}$ for $n=0,1$. This information is uniquely prescribed for a well-defined boundary value problem. The expressions in tables 1 and 2, and appendix A, thus eliminate the need to solve a system of differential equations for each flow problem and provide a complete asymptotic theory correct to $O\left(\theta^{-1}\right)$, in the limit of high frequency ratio and small scaled Knudsen number, i.e. $\theta \gg 1$ and $k \ll 1$. This theory complements previous analyses at (i) small scaled Knudsen number and low oscillation frequency $(k \ll 1$, $\theta \ll 1$ ) by Nassios \& Sader (2012) and Takata \& Hattori (2012), and (ii) high oscillation frequency, high Knudsen number and Strouhal numbers of order unity $(\theta \gg 1, K n \gg 1, S t \sim O(1))$ by Sone (2007). Next, we contrast these results to the formulation by Sone (2007).

\subsection{Comparison to the ultra-rarefied formulation by Sone (2007)}

As discussed in $\S 1$, for highly oscillatory flow in an ultra-rarefied gas, i.e. $\theta \gg 1$ and $k \gg 1$, inertia is balanced by gas particle advection in all spatial directions and the flow is free-molecular to leading order in $k^{-1}$; see Sone (2007) and $\S 2.1$. For weakly perturbed flows, this yields a collisionless Boltzmann equation and associated boundary condition, for the $n$ th-order term in the $k^{-1}$ expansion of the mass distribution function $\phi$ :

$$
\begin{gathered}
\frac{\partial \phi^{(0)}}{\partial t}+v_{i} \frac{\partial \phi^{(0)}}{\partial x_{i}}=0 \\
\left.\phi_{v_{i} n_{i} \geqslant 0}^{(0)}\right|_{b}=\sigma_{b}^{(0)}+2 V_{i}^{(0)} v_{i}+\left(v_{i}^{2}-\frac{3}{2}\right) \tau_{b}^{(0)} .
\end{gathered}
$$

In (3.28), all spatial directions are scaled isotropically by the geometric length scale $L \ll \lambda$, and particles incident on any solid wall undergo diffuse reflection. The Strouhal number $S t$ defined in (1.6) is assumed to be $O(1)$; see $\S 2.1$.

The leading-order reflected particle density $\sigma_{b}^{(0)}$ from a solid wall is a function of the mass distribution function of incident particles:

$$
\begin{aligned}
\sigma_{b}^{(0)}= & -\frac{1}{2} \tau_{b}^{(0)}-\left.2 \sqrt{\pi} \int_{-\infty}^{\infty} \int_{-\infty}^{\infty} \int_{-\infty}^{0} v_{i} n_{i} \phi_{v_{i} n_{i} \geqslant 0}^{(0)}\right|_{y=0} E\left(v_{i} t_{i}^{1}, v_{i} t_{i}^{2}, v_{i} n_{i}\right) \\
& \times \mathrm{d}\left(v_{i} n_{i}\right) \mathrm{d}\left(v_{i} t_{i}^{1}\right) \mathrm{d}\left(v_{i} t_{i}^{2}\right) .
\end{aligned}
$$

At higher order in $k^{-1}$, Sone (2007) showed that collisions become important, with $\phi^{(1)}$ given by

$$
\begin{gathered}
\frac{\partial \phi^{(1)}}{\partial t}+v_{i} \frac{\partial \phi^{(1)}}{\partial x_{i}}=\sigma^{(0)}-\phi^{(0)}+2 \bar{v}_{i}^{(0)} v_{i}+\left(v_{i}^{2}-\frac{3}{2}\right) \tau^{(0)}, \\
\left.\phi_{v_{i} n_{i} \geqslant 0}^{(1)}\right|_{b}=\sigma_{b}^{(1)}+2 V_{i}^{(1)} v_{i}+\left(v_{i}^{2}-\frac{3}{2}\right) \tau_{b}^{(1)},
\end{gathered}
$$




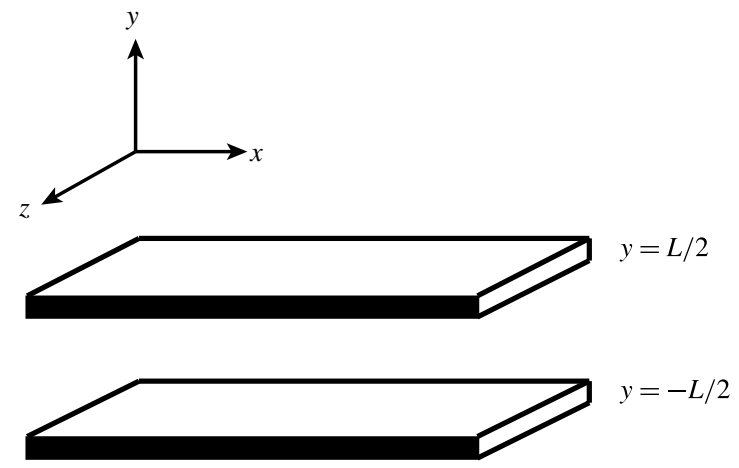

FIGURE 2. Schematic showing infinite parallel walls where temperature gradients are applied in the $x$-direction. Gas is confined between the walls.

where the reflected particle density $\sigma_{b}^{(1)}$ is

$$
\begin{aligned}
\sigma_{b}^{(1)}= & -\frac{1}{2} \tau_{b}^{(1)}-\left.2 \sqrt{\pi} \int_{-\infty}^{\infty} \int_{-\infty}^{\infty} \int_{-\infty}^{0} v_{i} n_{i} \phi_{v_{i} n_{i} \geqslant 0}^{(1)}\right|_{y=0} E\left(v_{i} t_{i}^{1}, v_{i} t_{i}^{2}, v_{i} n_{i}\right) \\
& \times \mathrm{d}\left(v_{i} n_{i}\right) \mathrm{d}\left(v_{i} t_{i}^{1}\right) \mathrm{d}\left(v_{i} t_{i}^{2}\right) .
\end{aligned}
$$

Importantly, (3.28) and (3.30) are valid throughout the gas, i.e. no boundary layer is present for $k \gg 1$ and $\theta \gg 1$; the reflected gas particle densities given in (3.29) and (3.31) are therefore coupled for problems involving more than one wall.

This differs markedly from the results for highly oscillatory flow at small scaled Knudsen number, i.e. $\theta \gg 1$ and $k \ll 1$, where wall-normal and tangential advection of gas particles have disproportionate effects on the flow near a solid wall. This is because particles can travel a short distance $L_{c} \ll \lambda$ away from a wall before undergoing a collision. A collisionless boundary layer is therefore present near any solid wall, where wall-normal advection balances inertia to leading order in $\theta^{-1}$; see $\S 3.4$. The collisionless Boltzmann equation does not appear within the collisionless layer, because tangential advection is a higher-order effect in $\theta^{-1}$ relative to both inertia and wall-normal advection. This physically distinguishes highly oscillatory flows in a slightly rarefied gas studied here, from the ultra-rarefied or free-molecular case investigated by Sone (2007).

\section{Application to time-varying thermal creep}

To illustrate the utility of the derived theory, we analyse the oscillatory thermal creep problem between two walls, which are infinite in extent and separated by a distance $L$; see figure 2 . In accordance with the presented theory, we implicitly assume that (i) the oscillation frequency greatly exceeds the inter-particle collision frequency of the gas $v$, i.e. $\theta \gg 1$, and (ii) the scaled Knudsen number is small, i.e. $k \ll 1$. Solutions to first order in the inverse frequency ratio $\theta^{-1}$ are presented. Oscillatory temperature gradients of magnitude $A$ in the $x$-direction are imposed at the walls, and no body force exists, i.e. $a_{i}=0$.

We consider two canonical flows: (i) the symmetric case, where temperature gradients are identical on both walls, and (ii) the antisymmetric case, where they are equal and opposite in sign. The general problem can be constructed from a 


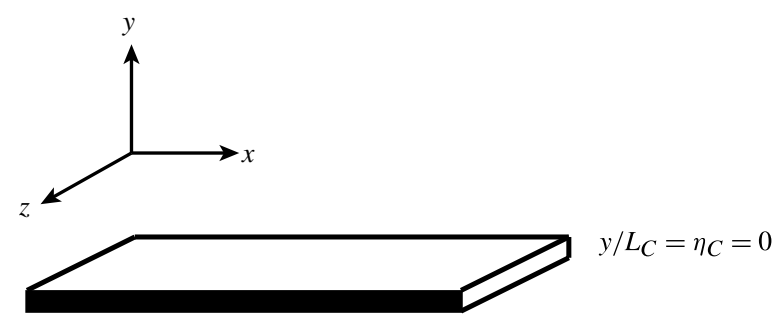

FIGURE 3. Schematic showing a single infinite plane wall, where a temperature gradient is applied in the $x$-direction. An infinite expanse of gas is located in the upper half-plane, $\eta_{C}>0$.

linear superposition of these flows, as in Nassios \& Sader (2012), who considered the complementary problem of low oscillation frequency.

From table 1, it is evident that no bulk flow exists for either the symmetric or antisymmetric oscillatory thermal creep problems. The only non-trivial flow occurs within the collisionless layers and is thus localized to the walls. Consequently, analysis of the flow at the top wall $(y=L / 2)$ formally decouples from that at the bottom wall $(y=-L / 2)$. It is therefore sufficient to analyse the thermal creep flow generated in a half-space of gas by an oscillatory temperature gradient imposed along an isolated plane wall; see figure 3. The flow between two parallel plane walls (the two-wall problem) is then constructed by an appropriate translation and linear superposition of the isolated wall solution.

Within the collisionless layer near a single wall, the scaled wall-normal coordinate is $\eta_{C}$, with the wall positioned at $\eta_{C}=0$. The length scale in the wall-normal direction is the most probable distance travelled by a particle over a single oscillation period $L_{c}$; see (2.12). The length scale $l$ in the $x$-direction is the reciprocal of the (scaled) wall temperature gradient; see figures 2 and 3 , and

$$
l \equiv\left(\frac{A}{T_{0}}\right)^{-1}
$$

As in the two-wall case, $A$ is the magnitude of the temperature gradient in the $x$-direction; see figure 3 . The temperature scale $T_{0}$ is the wall temperature at the reference position $x=0$; the wall temperature at this point is an appropriate scale because it remains constant for all time, as we now illustrate. The $n$ th-order terms in the $\theta^{-1}$-expansion of (perturbed) wall temperature $\tau_{b}$ are

$$
\begin{gathered}
\tau_{b}^{(0)}=x, \\
\tau_{b}^{(n)}=0, \quad n \geqslant 1,
\end{gathered}
$$

where $x$ is the scaled coordinate tangential to the wall; see figure 3. From (4.2), $\tau_{b}^{(n)}=0$ for all $n \geqslant 0$ at $x=0$, thus establishing $T_{0}$ as an appropriate temperature scale for all time. Also, the wall is stationary and therefore

$$
V_{i}^{(n)}=0,
$$

for all $n \geqslant 0$. In addition, we require expressions for the reflected particle densities at leading and first order, i.e. $\sigma_{b}^{(0)}$ and $\sigma_{b}^{(1)}$. Substituting (4.2) and (4.3), as well as the 
formulae for $\phi_{C \mid v_{y}<0}^{(0)}$ and $\phi_{C \mid v_{y}<0}^{(1)}$ from (3.14) and (3.24), into (2.23) yields

$$
\begin{gathered}
\sigma_{b}^{(0)}=-\frac{\tau_{b}^{(0)}}{2} \\
\sigma_{b}^{(1)}=-4 \sqrt{\pi} \tau_{b}^{(0)}\left(I_{3,1}(0)-I_{1,1}(0)+\frac{1}{3}\left[I_{4,2}(0)-\frac{3}{2} I_{2,2}(0)+\frac{3}{2} I_{0,2}(0)\right.\right. \\
\left.\left.-\frac{1}{2} I_{4,0}(0)+\frac{9}{4} I_{2,0}(0)-\frac{9}{4} I_{0,0}(0)\right]\right)
\end{gathered}
$$

The equations for the gas density, mean velocity, temperature and stress tensor of the gas in table 2 and appendix $A$ are defined in terms of $P_{b}^{(0)}=\sigma_{b}^{(0)}+\tau_{b}^{(0)}$ and $P_{b}^{(1)}=\sigma_{b}^{(1)}+\tau_{b}^{(1)}$, which we summarize in (4.5):

$$
\begin{gathered}
P_{b}^{(0)}=\frac{\tau_{b}^{(0)}}{2} \\
P_{b}^{(1)}=-4 \sqrt{\pi} \tau_{b}^{(0)}\left(I_{3,1}(0)-I_{1,1}(0)+\frac{1}{3}\left[I_{4,2}(0)-\frac{3}{2} I_{2,2}(0)+\frac{3}{2} I_{0,2}(0)\right.\right. \\
\left.\left.-\frac{1}{2} I_{4,0}(0)+\frac{9}{4} I_{2,0}(0)-\frac{9}{4} I_{0,0}(0)\right]\right) .
\end{gathered}
$$

Substituting (4.2), (4.3) and (4.5) into the expressions in table 2 and appendix A yields the complete solution for the gas density, mean tangential and normal velocities, and the temperature to $O\left(\theta^{-1}\right)$; these are summarized in table 5. The stress tensor components are given in table 6 .

\subsection{Discussion of the thermal creep flow}

The flow is localized to the wall and is driven by the leading-order wall temperature $\tau_{b}^{(0)}$; see (4.2). To ensure zero mass flux across the wall, the leading-order $(n=0)$ and first-order $(n=1)$ reflected gas particle densities at the wall are modified; see (4.4). Thus, rapid oscillations in the wall temperature at leading order induce perturbations to both the temperature and density of the gas away from the wall, which we discuss in $\S 4.1 .1$. In $\$ 4.1 .2$, we examine the mean wall-normal flows at leading and first order in $\theta^{-1}$, before studying the thermal creep flow in $\S 4.1 .3$, and the shear and normal stresses in the gas in $\$ \S 4.1 .5$ and 4.1.6, respectively. For clarity, all leading-order $(n=0)$ solutions will be plotted with solid lines, whereas the first-order results $(n=1)$ will be represented by dashed lines.

\subsubsection{Temperature and density fields}

Plots of the leading-order and first-order temperature, $\tau_{C}^{(0)} / x$ and $\tau_{C}^{(1)} / x$, are given in figure 4; both real and imaginary parts are presented. Note that the true (physical) solutions of this time-varying flow are given by a weighted superposition between the real and imaginary parts. For example, if the temperature at the wall is $T_{\text {true }}=\operatorname{Re}\{T \exp (-\mathrm{i} \omega t)\}$, then all true flow/temperature variables are given by $X_{\text {true }}=\operatorname{Re}\{X \exp (-\mathrm{i} \omega t)\}=\operatorname{Re}\{X\} \cos (\omega t)-\operatorname{Im}\{X\} \sin (\omega t)$, for any transport variable $X$. Thus, the solution periodically alternates between the real and imaginary components, as time evolves.

First consider the real component of the temperature. We remind the reader that the scaled temperature of the wall at leading order is $\tau_{b}^{(0)} / x=1$. In contrast, the leadingorder $(n=0)$ gas temperature (evaluated at the wall) is $\tau_{C}^{(0)} / x=0.5$; see figure $4(a)$. 


$$
\begin{aligned}
& \frac{\sqrt{\pi} \sigma_{C}^{(0)}}{x} \quad J_{2}\left(-\mathrm{i} \eta_{C}\right)-J_{0}\left(-\mathrm{i} \eta_{C}\right) \\
& \frac{3}{2} \frac{\sqrt{\pi} \tau_{C}^{(0)}}{x} \quad J_{4}\left(-\mathrm{i} \eta_{C}\right)-\frac{3}{2} J_{2}\left(-\mathrm{i} \eta_{C}\right)+\frac{3}{2} J_{0}\left(-\mathrm{i} \eta_{C}\right) \\
& \begin{array}{ll}
\frac{\sqrt{\pi} \bar{v}_{C \mid y}^{(0)}}{x} & J_{3}\left(-\mathrm{i} \eta_{C}\right)-J_{1}\left(-\mathrm{i} \eta_{C}\right) \\
\bar{v}_{C \mid x}^{(0)} & 0
\end{array} \\
& 2\left\{\left[I_{3,0}\left(\eta_{C}\right)-I_{1,0}\left(\eta_{C}\right)\right]+\frac{1}{3}\left[I_{4,1}\left(\eta_{C}\right)-\frac{3}{2} I_{2,1}\left(\eta_{C}\right)+\frac{3}{2} I_{0,1}\left(\eta_{C}\right)\right.\right. \\
& \left.\left.-\frac{1}{2} I_{4,-1}\left(\eta_{C}\right)+\frac{9}{4} I_{2,-1}\left(\eta_{C}\right)-\frac{9}{4} I_{0,-1}\left(\eta_{C}\right)\right]\right\}-\frac{\eta_{C}}{\sqrt{\pi}}\left[J_{1}\left(-\mathrm{i} \eta_{C}\right)\right. \\
& \begin{array}{ll}
\sigma_{C}^{(1)} & \left.-J_{-1}\left(-\mathrm{i} \eta_{C}\right)\right]-4 J_{0}\left(-\mathrm{i} \eta_{C}\right)\left(I_{3,1}(0)-I_{1,1}(0)+\frac{1}{3}\left[I_{4,2}(0)-\frac{3}{2} I_{2,2}(0)\right.\right.
\end{array} \\
& \left.\left.+\frac{3}{2} I_{0,2}(0)-\frac{1}{2} I_{4,0}(0)+\frac{9}{4} I_{2,0}(0)-\frac{9}{4} I_{0,0}(0)\right]\right) \\
& 2\left\{\left[I_{3,2}\left(\eta_{C}\right)-I_{1,2}\left(\eta_{C}\right)-\frac{1}{2} I_{3,0}\left(\eta_{C}\right)+\frac{1}{2} I_{1,0}\left(\eta_{C}\right)\right]+\frac{1}{3}\left[I_{4,3}\left(\eta_{C}\right)\right.\right. \\
& -\frac{3}{2} I_{2,3}\left(\eta_{C}\right)+\frac{3}{2} I_{0,3}\left(\eta_{C}\right)-I_{4,1}\left(\eta_{C}\right)+3 I_{2,1}\left(\eta_{C}\right)-3 I_{0,1}\left(\eta_{C}\right)+\frac{5}{4} I_{4,-1}\left(\eta_{C}\right) \\
& \left.\left.\frac{3}{2} \frac{\tau_{C}^{(1)}}{x} \quad-\frac{21}{8} I_{2,-1}\left(\eta_{C}\right)+\frac{21}{8} I_{0,-1}\left(\eta_{C}\right)\right]\right\}-\frac{\eta_{C}}{\sqrt{\pi}}\left[J_{3}\left(-\mathrm{i} \eta_{C}\right)-\frac{3}{2} J_{1}\left(-\mathrm{i} \eta_{C}\right)\right. \\
& \left.+\frac{3}{2} J_{-1}\left(-\mathrm{i} \eta_{C}\right)\right]-4\left(J_{2}\left(-\mathrm{i} \eta_{C}\right)-\frac{1}{2} J_{0}\left(-\mathrm{i} \eta_{C}\right)\right)\left(I_{3,1}(0)-I_{1,1}(0)\right. \\
& \left.+\frac{1}{3}\left[I_{4,2}(0)-\frac{3}{2} I_{2,2}(0)+\frac{3}{2} I_{0,2}(0)-\frac{1}{2} I_{4,0}(0)+\frac{9}{4} I_{2,0}(0)-\frac{9}{4} I_{0,0}(0)\right]\right) \\
& 2\left\{\left[I_{3,1}\left(\eta_{C}\right)-I_{1,1}\left(\eta_{C}\right)\right]+\frac{1}{3}\left[I_{4,2}\left(\eta_{C}\right)-\frac{3}{2} I_{2,2}\left(\eta_{C}\right)+\frac{3}{2} I_{0,2}\left(\eta_{C}\right)\right.\right. \\
& \left.\left.-\frac{1}{2} I_{4,0}\left(\eta_{C}\right)+\frac{9}{4} I_{2,0}\left(\eta_{C}\right)-\frac{9}{4} I_{0,0}\left(\eta_{C}\right)\right]\right\}-\frac{\eta_{C}}{\sqrt{\pi}}\left[J_{2}\left(-\mathrm{i} \eta_{C}\right)\right. \\
& \left.-J_{0}\left(-\mathrm{i} \eta_{C}\right)\right]-4 J_{1}\left(-\mathrm{i} \eta_{C}\right)\left(I_{3,1}(0)-I_{1,1}(0)+\frac{1}{3}\left[I_{4,2}(0)-\frac{3}{2} I_{2,2}(0)\right.\right. \\
& \left.\left.+\frac{3}{2} I_{0,2}(0)-\frac{1}{2} I_{4,0}(0)+\frac{9}{4} I_{2,0}(0)-\frac{9}{4} I_{0,0}(0)\right]\right) \\
& \frac{\bar{v}_{C \mid x}^{(1)}}{k} \quad-\frac{\eta_{C}}{2 \sqrt{\pi}}\left(J_{1}\left(-\mathrm{i} \eta_{C}\right)+J_{-1}\left(-\mathrm{i} \eta_{C}\right)\right)
\end{aligned}
$$

TABLE 5. The hydrodynamic quantities, $\sigma, \tau$ and $\bar{v}_{i}$, at leading and first order in the inverse frequency ratio $\theta^{-1}$ i.e. $n=0$ and $n=1$, for the oscillatory thermal creep problem across an infinite plane wall.

This represents a temperature jump at the wall. Interestingly, the first-order $(n=1)$ gas temperature displays no jump at the wall, matching the first-order wall temperature, which is zero; see (4.2) and figure 4(a). 


$$
\begin{aligned}
& \epsilon_{C \mid z y}^{(0)}=\epsilon_{C \mid y z}^{(0)} \quad 0 \\
& \epsilon_{C \mid x z}^{(0)}=\epsilon_{C \mid z x}^{(0)} \quad 0 \\
& \epsilon_{C \mid x y}^{(0)}=\epsilon_{C \mid y x}^{(0)} \quad 0 \\
& \frac{\sqrt{\pi}}{2} \frac{\epsilon_{C \mid y y}^{(0)}}{x} \quad J_{4}\left(-\mathrm{i} \eta_{C}\right)-J_{2}\left(-\mathrm{i} \eta_{C}\right) \\
& \frac{\sqrt{\pi} \epsilon_{C \mid x x}^{(0)}}{x}=\frac{\sqrt{\pi} \epsilon_{C \mid z z}^{(0)}}{x} \quad J_{2}\left(-\mathrm{i} \eta_{C}\right) \\
& \epsilon_{C \mid y z}^{(1)}=\epsilon_{C \mid z y}^{(1)} \quad 0 \\
& \epsilon_{C \mid x z}^{(1)}=\epsilon_{C \mid x x}^{(1)} \quad 0 \\
& \frac{\epsilon_{C \mid x y}^{(1)}}{k}=\frac{\epsilon_{C \mid y x}^{(1)}}{k} \quad-\frac{\eta_{C}}{\sqrt{\pi}}\left[J_{2}\left(-\mathrm{i} \eta_{C}\right)+J_{0}\left(-\mathrm{i} \eta_{C}\right)\right] \\
& 2\left\{I_{3,2}\left(\eta_{C}\right)-I_{1,2}\left(\eta_{C}\right)+\frac{1}{3}\left[I_{4,3}\left(\eta_{C}\right)-\frac{3}{2} I_{2,3}\left(\eta_{C}\right)+\frac{3}{2} I_{0,3}\left(\eta_{C}\right)\right.\right. \\
& \left.\left.-\frac{1}{2} I_{4,1}\left(\eta_{C}\right)+\frac{9}{4} I_{2,1}\left(\eta_{C}\right)-\frac{9}{4} I_{0,1}\left(\eta_{C}\right)\right]\right\}-\frac{\eta_{C}}{\sqrt{\pi}}\left[J_{3}\left(-\mathrm{i} \eta_{C}\right)\right. \\
& \frac{1}{2} \frac{\epsilon_{C \mid y y}^{(1)}}{x} \\
& \left.-J_{1}\left(-\mathrm{i} \eta_{C}\right)\right]-4 J_{2}\left(-\mathrm{i} \eta_{C}\right)\left(I_{3,1}(0)-I_{1,1}(0)+\frac{1}{3}\left[I_{4,2}(0)-\frac{3}{2} I_{2,2}(0)\right.\right. \\
& \left.\left.+\frac{3}{2} I_{0,2}(0)-\frac{1}{2} I_{4,0}(0)+\frac{9}{4} I_{2,0}(0)-\frac{9}{4} I_{0,0}(0)\right]\right) \\
& 2\left\{I_{3,0}\left(\eta_{C}\right)-I_{1,0}\left(\eta_{C}\right)+\frac{1}{3}\left[I_{4,1}\left(\eta_{C}\right)-\frac{3}{2} I_{2,1}\left(\eta_{C}\right)+\frac{3}{2} I_{0,1}\left(\eta_{C}\right)\right.\right. \\
& \left.\left.+\frac{1}{2} I_{4,-1}\left(\eta_{C}\right)+\frac{3}{4} I_{2,-1}\left(\eta_{C}\right)-\frac{3}{4} I_{0,-1}\left(\eta_{C}\right)\right]\right\}-\frac{\eta_{C}}{\sqrt{\pi}} J_{1}\left(-\mathrm{i} \eta_{C}\right) \\
& -4 J_{0}\left(-\mathrm{i} \eta_{C}\right)\left(I_{3,1}(0)-I_{1,1}(0)+\frac{1}{3}\left[I_{4,2}(0)-\frac{3}{2} I_{2,2}(0)+\frac{3}{2} I_{0,2}(0)\right.\right. \\
& \left.\left.-\frac{1}{2} I_{4,0}(0)+\frac{9}{4} I_{2,0}(0)-\frac{9}{4} I_{0,0}(0)\right]\right)
\end{aligned}
$$

TABLE 6. The stress tensor components, $\epsilon_{i j}$, at leading and first order in the inverse frequency ratio $\theta^{-1}$ i.e. $n=0$ and $n=1$, for the oscillatory thermal creep problem across an infinite plane wall.

In contrast, the imaginary component of the leading-order $(n=0)$ gas temperature in figure $4(b)$ exhibits no jump, while the first-order $(n=1)$ correction is non-zero at the wall. Thus, the real component of the gas temperature at the wall is not altered at first order, while the imaginary component is modified - the real and imaginary components exhibit opposite behaviour. This establishes that as time evolves in a real flow, a temperature jump is exhibited at all time but alternates between a leading-order and first-order effect in $\theta^{-1}$.

With this knowledge in hand, we now probe the underlying physics driving the first-order temperature jump (in the imaginary component). To proceed, we consider the three physical components affecting this behaviour: free-molecular flow, collisional effects and advection parallel to the wall. Importantly, the advective component 
(a)

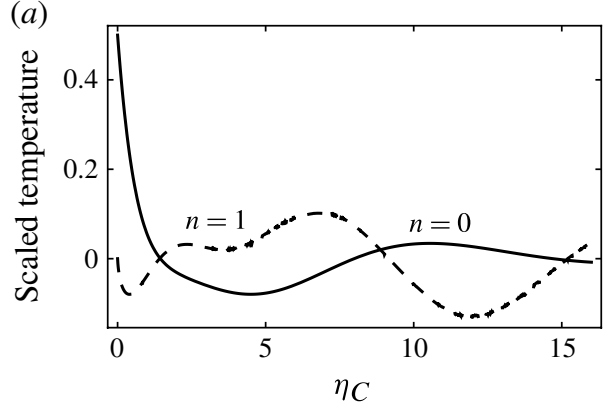

(b)

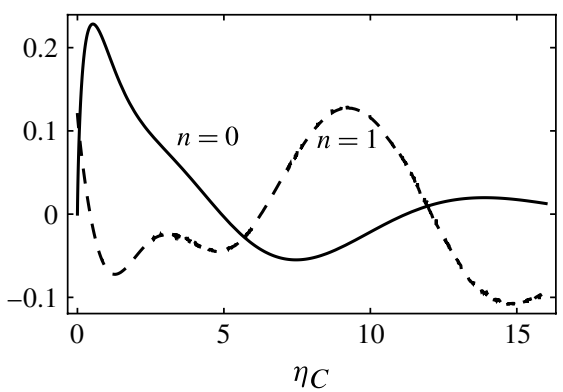

FIGURE 4. Scaled temperature, $\tau_{C}^{(0)} / x$ and $\tau_{C}^{(1)} / x$ : (a) real component; (b) imaginary component. Results given for the leading-order solution $(n=0$, solid) and first-order correction $(n=1$, dashed).

(parallel to the wall in the $x$-direction) of the Boltzmann-BGK equation is an odd function of the tangential particle velocity $v_{x}$ and therefore does not affect the gas temperature. This is evident from (3.23) and (3.27) $-K^{(1)}$ is an odd function of the tangential particle velocity $v_{x}$ for flow over a stationary and flat wall, leading to $\phi_{C, t a n}^{(1)}$ also being an odd function of $v_{x}$. In contrast, free-molecular and collisional contributions to the first-order mass distribution function are even functions of the tangential particle velocity, $v_{x}$, and thus provide non-zero contributions to the firstorder gas temperature field; see (3.25) and (3.26).

We now compare the relative contribution of these non-zero effects. The first-order $(n=1)$ temperature is formally expanded (due to linearity):

$$
\frac{\tau_{C}^{(1)}}{x}=\frac{\tau_{C, f m}^{(1)}}{x}+\frac{\tau_{C, c o l}^{(1)}}{x}
$$

where

$$
\begin{aligned}
\frac{\tau_{C, f m}^{(1)}}{x}= & -\frac{8}{3 \pi}\left(J_{2}\left(-\mathrm{i} \eta_{C}\right)-\frac{1}{2} J_{0}\left(-\mathrm{i} \eta_{C}\right)\right)\left(I_{3,1}(0)-I_{1,1}(0)\right. \\
& \left.+\frac{1}{3}\left[I_{4,2}(0)-\frac{3}{2} I_{2,2}(0)+\frac{3}{2} I_{0,2}(0)-\frac{1}{2} I_{4,0}(0)+\frac{9}{4} I_{2,0}(0)-\frac{9}{4} I_{0,0}(0)\right]\right), \\
\frac{\tau_{C, c o l}^{(1)}}{x}= & \frac{4}{3 \pi}\left[\left[I_{3,2}\left(\eta_{C}\right)-I_{1,2}\left(\eta_{C}\right)-\frac{1}{2} I_{3,0}\left(\eta_{C}\right)+\frac{1}{2} I_{1,0}\left(\eta_{C}\right)\right]+\frac{1}{3}\left[I_{4,3}\left(\eta_{C}\right)\right.\right. \\
& -\frac{3}{2} I_{2,3}\left(\eta_{C}\right)+\frac{3}{2} I_{0,3}\left(\eta_{C}\right)-I_{4,1}\left(\eta_{C}\right)+3 I_{2,1}\left(\eta_{C}\right)-3 I_{0,1}\left(\eta_{C}\right) \\
& \left.\left.+\frac{5}{4} I_{4,-1}\left(\eta_{C}\right)-\frac{21}{8} I_{2,-1}\left(\eta_{C}\right)+\frac{21}{8} I_{0,-1}\left(\eta_{C}\right)\right]\right]-\frac{2}{3} \frac{\eta_{C}}{\sqrt{\pi}}\left[J_{3}\left(-\mathrm{i} \eta_{C}\right)\right. \\
& \left.-\frac{3}{2} J_{1}\left(-\mathrm{i} \eta_{C}\right)+\frac{3}{2} J_{-1}\left(-\mathrm{i} \eta_{C}\right)\right] .
\end{aligned}
$$

Plots of the real and imaginary components of $\tau_{C, f m}^{(1)} / x$ and $\tau_{C, \text { col }}^{(1)} / x$ are given in figure 5. Note that the free-molecular contribution exhibits classical wave behaviour. Nonetheless, the collisional term $\tau_{C, \text { col }}^{(1)} / x$ is an order of magnitude larger than this free-molecular contribution $\tau_{C, f m}^{(1)} / x$, and thus wave behaviour has a minimal effect on 


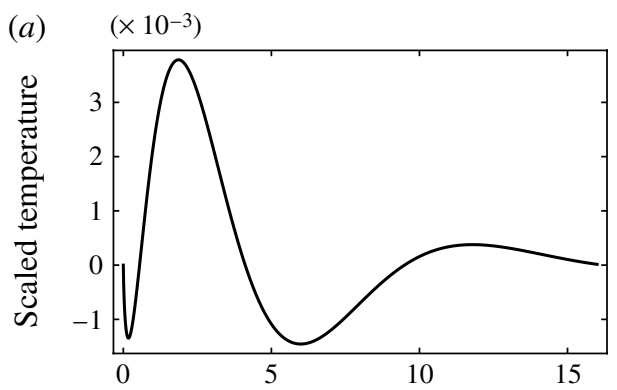

(b)
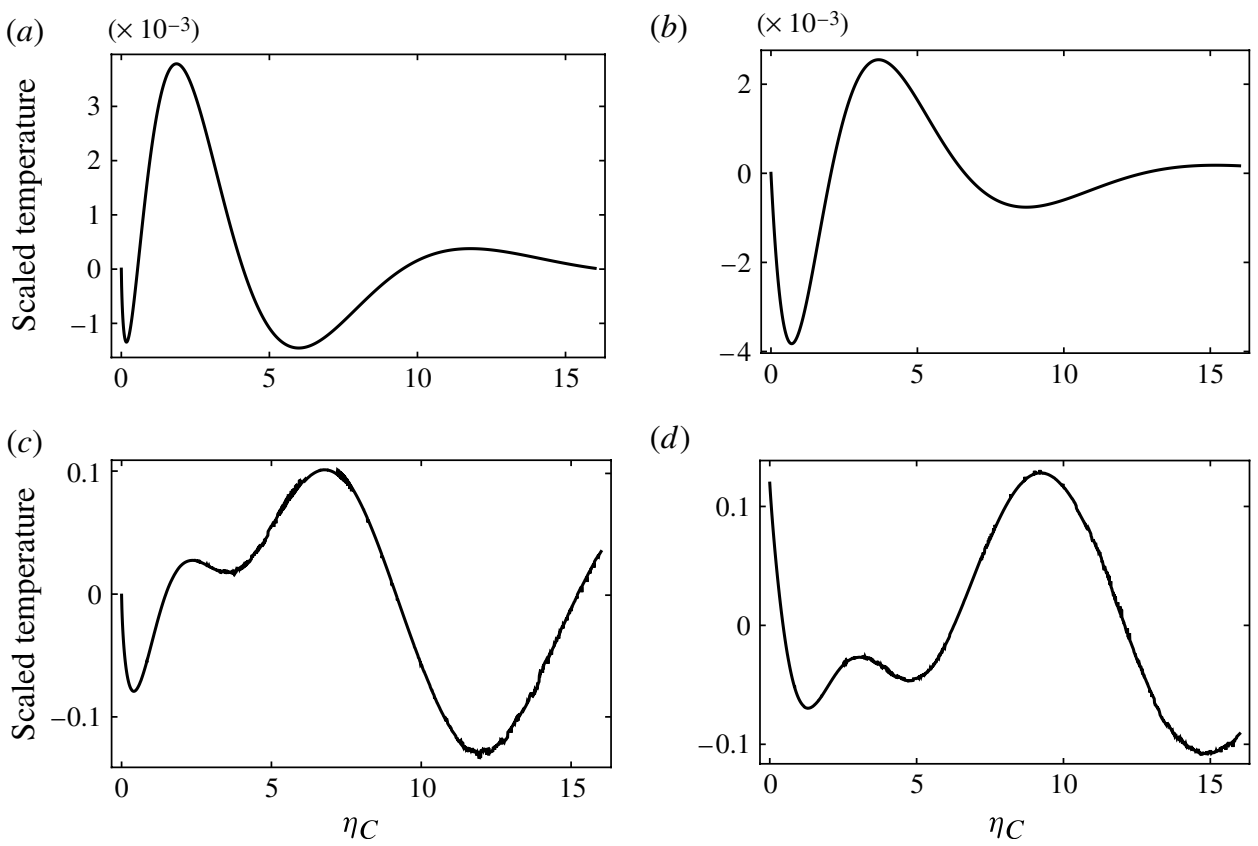

(d)

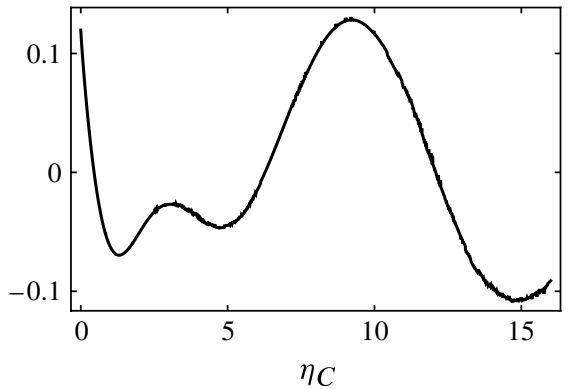

FIGURE 5. The free-molecular and collisional components of the first-order scaled temperature, $\tau_{C}^{(1)} / x$. Free-molecular component: $(a)$ real; $(b)$ imaginary. Collisional component: $(c)$ real; $(d)$ imaginary.

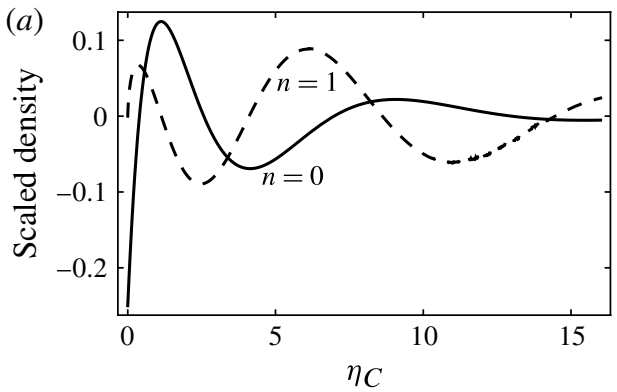

(b)

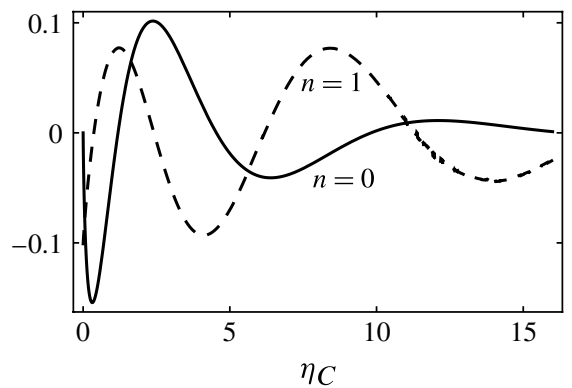

FigurE 6. Scaled density, $\sigma_{C}^{(0)} / x$ and $\sigma_{C}^{(1)} / x$ : $(a)$ real component; $(b)$ imaginary component. Results given for the leading-order solution $(n=0$, solid) and first-order correction $(n=1$, dashed).

the gas temperature. Furthermore, only the imaginary component of the collisional term exhibits a temperature jump. This establishes that collisional effects drive the first-order temperature jump which appears only in the imaginary component.

Plots of the scaled density at leading order and first order, $\sigma_{C}^{(0)} / x$ and $\sigma_{C}^{(1)} / x$, are given in figure 6. As for the temperature, the leading-order component of the density displays classical wave-like behaviour, as noted previously by Manela \& Hadjiconstantinou (2007), with collisional effects again dominating at first order; this can be easily seen by following a similar analysis to the above. 
(a)

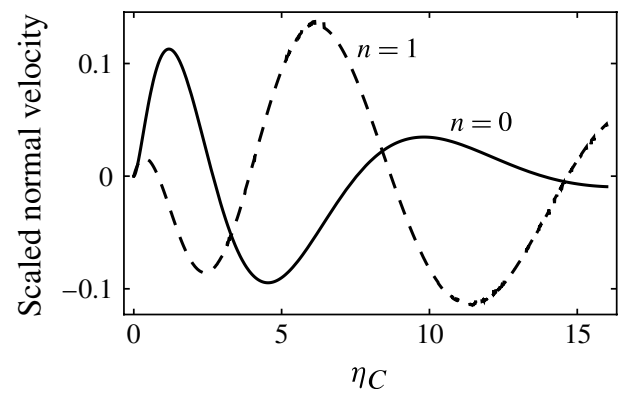

(b)

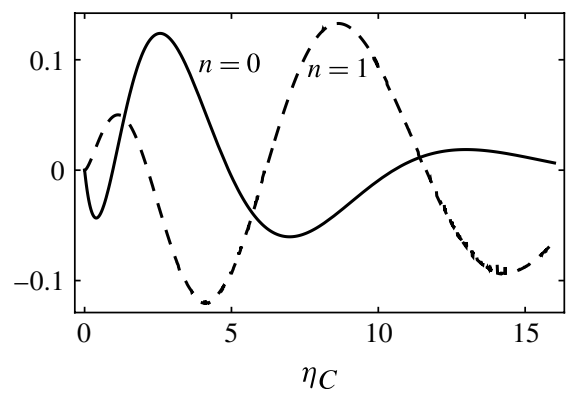

FIGURE 7. Scaled mean normal velocity, $\bar{v}_{C \mid y}^{(0)} / x$ and $\bar{v}_{C \mid y}^{(1)} / x$ : (a) real component; $(b)$ imaginary component. Results given for the leading-order solution ( $n=0$, solid) and firstorder correction $(n=1$, dashed).

\subsubsection{Mean normal velocity}

The scaled leading-order and first-order mean normal velocities, $\bar{v}_{C \mid y}^{(0)} / x$ and $\bar{v}_{C \mid y}^{(1)} / x$, are given in figure 7 . Since each component is proportional to the tangential coordinate $x$, these scaled mean velocities are independent of $x$. Note that the mean normal velocity is zero to $O\left(\theta^{-1}\right)$ for all positions away from the wall, $\eta_{C}$, at the reference point $x=0$.

The mean normal velocity is zero at the walls as required by no penetration. The leading-order flow is free-molecular and exhibits classical wave-like behaviour, as found for the temperature and density fields. To study the first-order component of the normal velocity, we follow a procedure identical to that in $\S 4.1 .1$. We thus express the mean normal velocity at this order as

$$
\frac{\bar{v}_{C \mid y}^{(1)}}{x}=\frac{\bar{v}_{C \mid y, f m}^{(1)}}{x}+\frac{\bar{v}_{C \mid y, c o l}^{(1)}}{x},
$$

where

$$
\begin{aligned}
\frac{\bar{v}_{C \mid y, f m}^{(1)}=-}{x}= & \frac{4}{\pi} J_{1}\left(-\mathrm{i} \eta_{C}\right)\left(I_{3,1}(0)-I_{1,1}(0)+\frac{1}{3}\left[I_{4,2}(0)-\frac{3}{2} I_{2,2}(0)+\frac{3}{2} I_{0,2}(0)\right.\right. \\
& \left.\left.-\frac{1}{2} I_{4,0}(0)+\frac{9}{4} I_{2,0}(0)-\frac{9}{4} I_{0,0}(0)\right]\right) \\
\frac{\bar{v}_{C \mid y, c o l}^{(1)}=}{x}= & \frac{2}{\pi}\left(\left[I_{3,1}\left(\eta_{C}\right)-I_{1,1}\left(\eta_{C}\right)\right]+\frac{1}{3}\left[I_{4,2}\left(\eta_{C}\right)-\frac{3}{2} I_{2,2}\left(\eta_{C}\right)+\frac{3}{2} I_{0,2}\left(\eta_{C}\right)\right.\right. \\
& \left.\left.-\frac{1}{2} I_{4,0}\left(\eta_{C}\right)+\frac{9}{4} I_{2,0}\left(\eta_{C}\right)-\frac{9}{4} I_{0,0}\left(\eta_{C}\right)\right]\right) \\
& -\frac{\eta_{C}}{\sqrt{\pi}}\left[J_{2}\left(-\mathrm{i} \eta_{C}\right)-J_{0}\left(-\mathrm{i} \eta_{C}\right)\right] .
\end{aligned}
$$

As proved for the temperature and density fields at this order, advection parallel to the wall does not affect the mean normal velocity because $\phi_{C, \tan }^{(1)}$ is an odd function of $v_{x}$.

Plots of the real and imaginary parts of $\bar{v}_{C \mid y, f m}^{(1)} / x$ and $\bar{v}_{C \mid y, c o l}^{(1)} / x$ are given in figure 8 . Figures $8(a)$ and $8(c)$ show that the real part of the collisional term is an order of magnitude larger than the free-molecular component. In contrast, the imaginary 


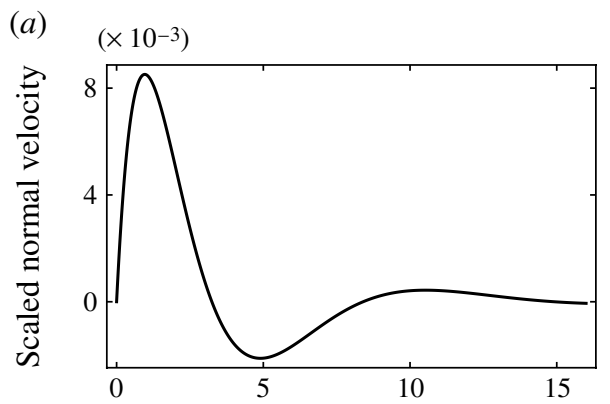

(b)

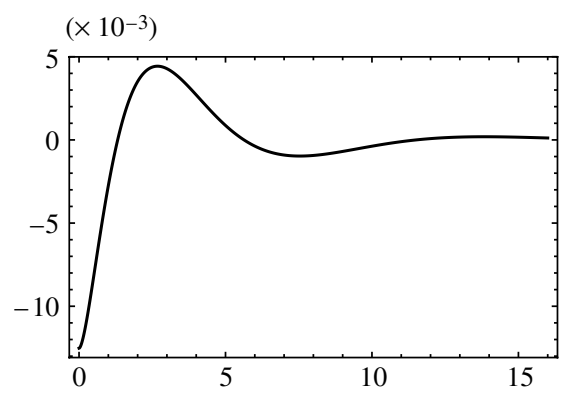

(c)

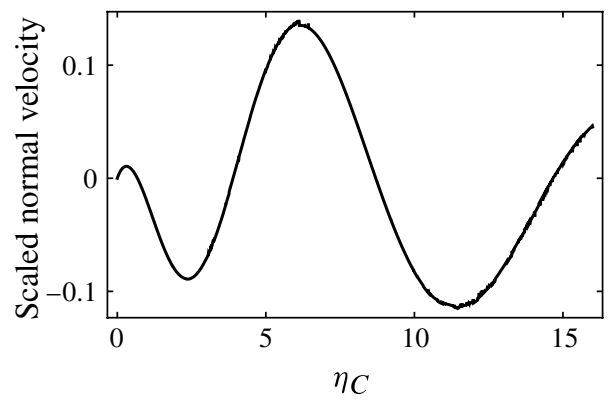

$(d)$

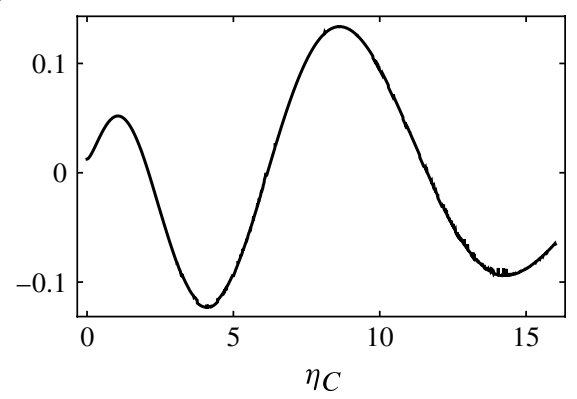

FIGURE 8. The free-molecular and collisional components of the first-order scaled mean normal velocity, $\bar{v}_{C \mid y}^{(1)} / x$. Free-molecular component: $(a)$ real; $(b)$ imaginary. Collisional component: $(c)$ real; $(d)$ imaginary.

parts are comparable in magnitude near the wall; at the wall, they are equivalent in magnitude and opposite in sign: see figures $8(b)$ and $8(d)$. Therefore, as time evolves, collisional and free-molecular effects periodically balance near the wall. This balance ensures that the impermeability condition is satisfied at first order for all time, and is distinct from our findings for the gas temperature and density.

\subsubsection{Mean tangential velocity}

Next, we examine thermal creep, i.e. the mean tangential velocity, induced by the high frequency oscillatory wall temperature gradients. In a slightly rarefied gas, $k \ll 1$, the leading-order $(n=0)$ mean tangential velocity in the $x$-direction $\bar{v}_{C \mid x}^{(0)}$ is zero; see table 5. In contrast, the scaled first-order mean tangential velocity $\bar{v}_{C \mid x}^{(1)} / k$ is non-zero; see table 5 and plots of the real and imaginary parts in figure 9 . Note that scalings for the normal and tangential velocities differ - the normal component is linearly dependent on $x$ whereas the tangential velocity is independent of $x$.

The first-order mean tangential velocity $\bar{v}_{C \mid x}^{(1)} / k$ is the leading-order thermal creep flow in a slightly rarefied gas at high oscillation frequency, i.e. $k \ll 1$ and $\theta \gg 1$. The real and imaginary parts exhibit wave-like behaviour in the normal coordinate $\eta_{C}$, with periodic zeros appearing for increasing $\eta_{C}$.

The physical mechanism driving the thermal creep flow is advection parallel to the wall. It can be shown using an analysis similar to $\$ 4.1 .1$ that collisional and free-molecular contributions are identically zero. This proof involves comparison of even and odd contributions in the particle velocity $v_{x}$. Interestingly, no slip is also observed between the mean gas velocity and the wall velocity, for both the real and imaginary components; see figure 9 . 


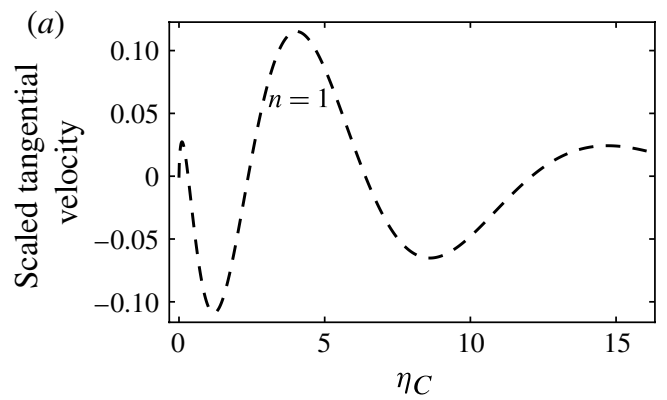

(b)

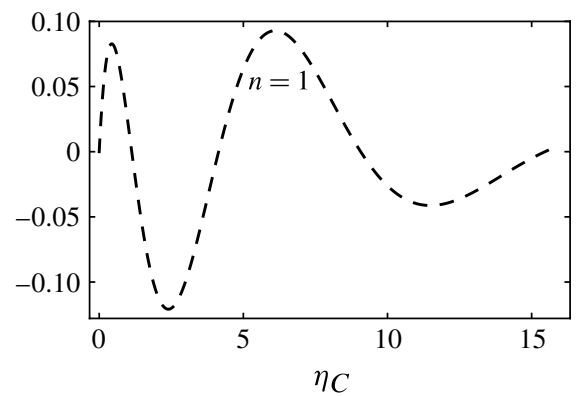

FIGURE 9. Scaled mean tangential velocity, $\bar{v}_{C \mid x}^{(1)} / k$ : (a) real component; $(b)$ imaginary component. Result given for the first-order correction $(n=1$, dashed). The leading-order solution $(n=0)$ is uniquely zero.
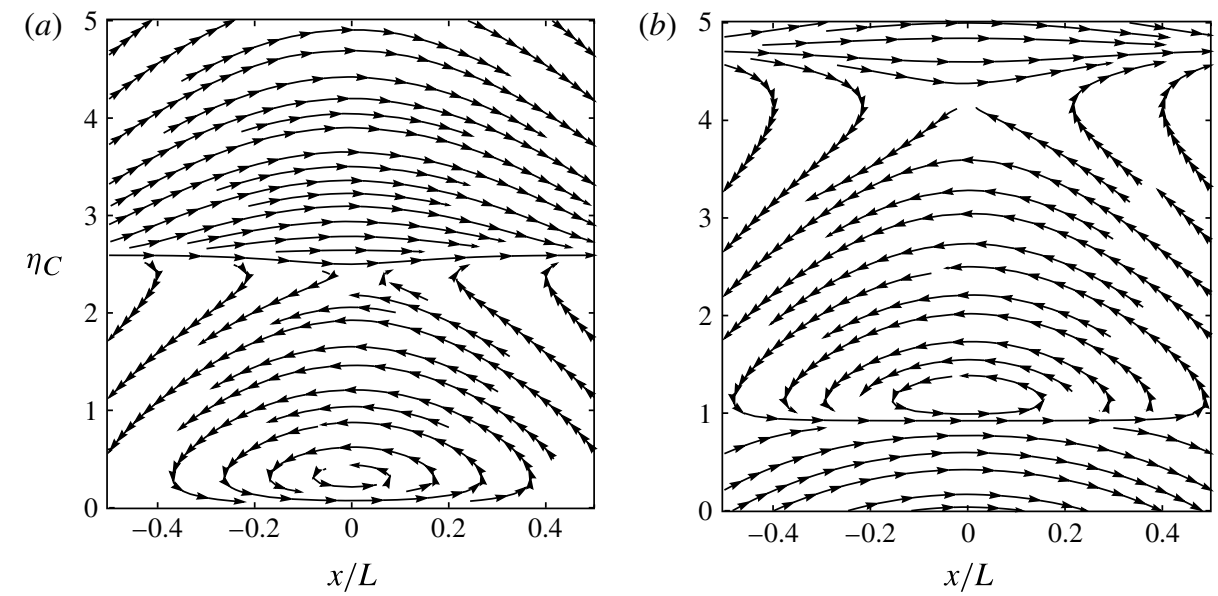

FIGURE 10. Streamlines for the flow at $\theta^{-1}=0.1$. The tangential velocity $\bar{v}_{C \mid x}$ has been scaled by $k$ to emphasize the critical points in the flow. Flow centred at $x=0:(a)$ real; $(b)$ imaginary.

\subsubsection{Streamlines}

The imposed temperature field at the walls generates a rich flow distribution in the gas. A series of critical points arise in the form of both centres and saddle points. These features are now illustrated and their origin explored.

From $\S \S 4.1 .2$ and 4.1.3, the mean normal velocity to $O\left(\theta^{-1}\right)$ is zero for all $\eta_{C}$, at the reference point $x=0$. In contrast, the mean tangential velocity exhibits a periodic array of zeros as $\eta_{C}$ increases, at $x=0$. This periodic array arises from the wave-like nature of this component; see $\S 4.1 .3$. This immediately generates a series of critical points along $x=0$, which coincide with the zeros of $\bar{v}_{C \mid x}^{(1)}$.

This phenomenon is clearly evident in figure 10, where the streamlines of this flow are presented. Note that these streamlines have been scaled by the Knudsen number to ensure they are universally valid for all (small) Knudsen number. This is achieved as follows. Because the mean tangential velocity is proportional to the scaled Knudsen number, $k$, it has been normalized by $k$, thus removing the Knudsen number dependence of this component. In contrast, the mean normal velocity is not scaled in this fashion. Taking these two components and plotting their streamlines gives the required universal solution. 


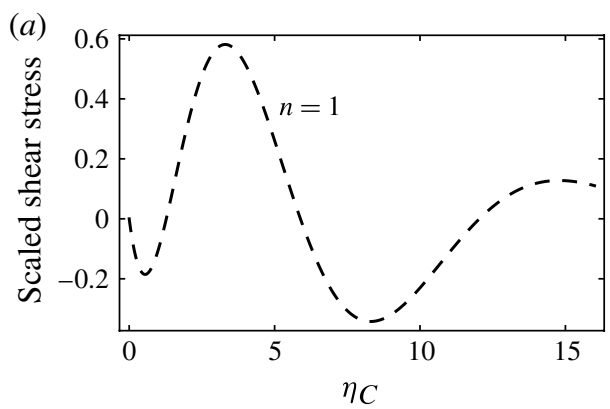

(b)

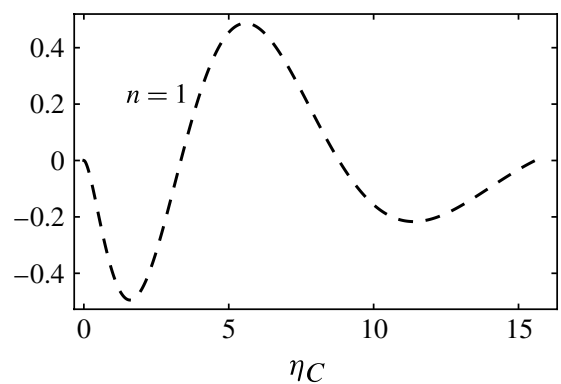

FIGURE 11. Scaled shear stress, $\epsilon_{C \mid x y}^{(1)} /(k)=\epsilon_{C \mid y x}^{(1)} /(k)$ : (a) real component; $(b)$ imaginary component. Result given for the first-order correction $(n=1$, dashed). The leading-order solution $(n=0)$ is uniquely zero.

\subsubsection{Shear stress}

We now examine the shear stress in the gas. To leading order $(n=0)$, all shear stress components are zero; see table 6. In contrast, the first-order $(n=1)$ scaled components $\epsilon_{C \mid x y}^{(1)} / k$ and $\epsilon_{C \mid y x}^{(1)} / k$ are non-zero, and are identical by symmetry. Plots of the real and imaginary parts of these components are given in figure 11. The shear stress is always zero at the wall, as required for a stationary wall; see table 4 .

The functions $\epsilon_{C \mid x y}^{(1)} / k$ and $\epsilon_{C \mid y x}^{(1)} / k$ are the leading-order shear stress components in the gas, and are of equivalent order in $\theta^{-1}$ to the thermal creep velocity field; see $\S 4.1 .3$. As for the velocity field, the shear stress components in the gas also have their genesis in advection of gas particles parallel to the wall - this can be proved using the methods outlined in $\S 4.1 .3$.

\subsubsection{Normal stresses}

In contrast to the shear stress, the normal stress components in the gas occur at both leading order $(n=0)$ and first order $(n=1)$. To study each component, we define $\hat{x}$ to be the unit vector in the $x$-direction, with similar interpretations for $\hat{y}$ and $\hat{z}$; see figure 3 .

The $\hat{x} \hat{x}$ and $\hat{z} \hat{z}$ normal stress components are identical. Plots of their real and imaginary parts, at leading $(n=0)$ and first order $(n=1)$ in $\theta^{-1}$, are given in figure $12(a, b)$. The $\hat{y} \hat{y}$ component differs from the other normal components, which is evident from figure $12(c, d)$.

Qualitative features of all normal stress components in figure 12, with respect to variations in position $\eta_{C}$ and time $t$, are similar to the gas temperature and density fields discussed in $\S 4.1 .1$.

\section{Conclusions}

We have investigated the high frequency oscillatory (time-varying) flow of a slightly rarefied gas, i.e. $\theta \gg 1$ and $k \ll 1$. This analysis complements previous theoretical investigations of (i) highly rarefied flows at high frequency by Sone (2007), i.e. $k \gg 1$ and $\theta \gg 1$, and (ii) slightly rarefied flows at low frequency by Nassios \& Sader (2012) and Takata \& Hattori (2012), i.e. $\theta \ll 1$ and $k \ll 1$. The geometry of any bounding wall was chosen to be arbitrary and smooth, with diffuse reflection of particles assumed.

A matched asymptotic expansion in the (small) inverse frequency ratio $\theta^{-1}$ was performed. In a slightly rarefied gas where $k \ll 1$, a local boundary layer flow near the 
(a)

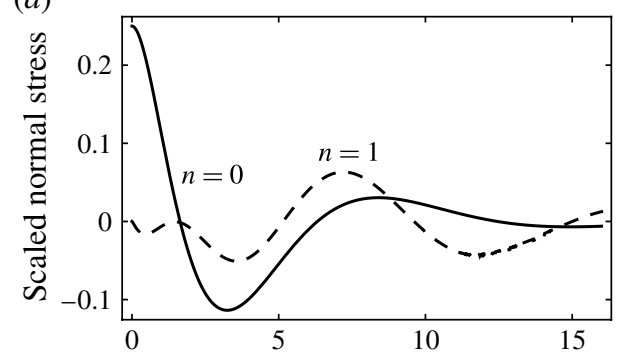

(c)

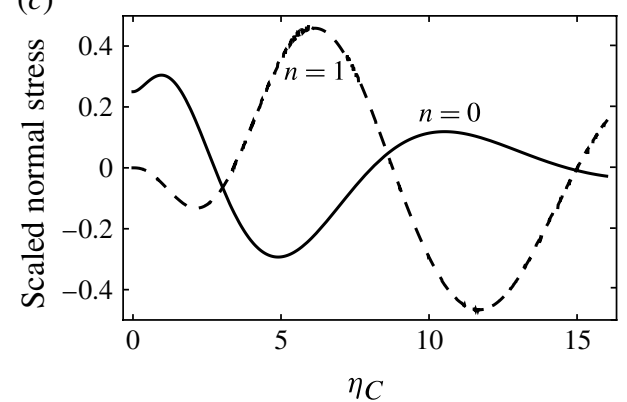

(b)

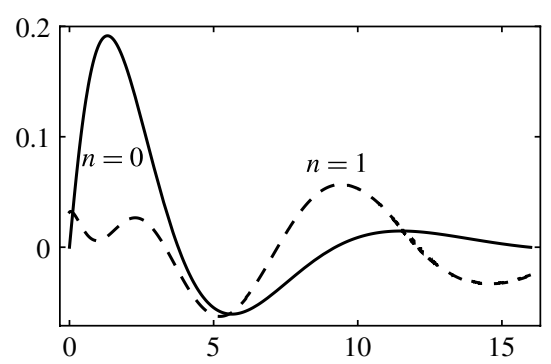

(d)

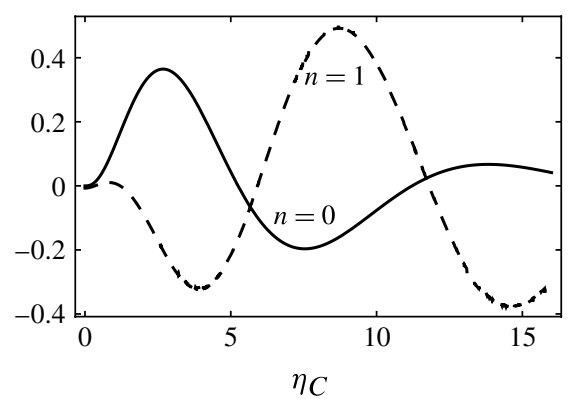

FIGURE 12. Scaled normal stresses, $\epsilon_{C \mid x x}^{(0)} / x$ and $\epsilon_{C \mid x x}^{(1)} / x$ : (a) real component; $(b)$ imaginary component. $\epsilon_{C \mid z z}^{(0)} / x$ and $\epsilon_{C \mid z z}^{(1)} / x$ are identical. $\epsilon_{C \mid y y}^{(0)} / x$ and $\epsilon_{C \mid y y}^{(1)} / x$ : (c) real component; $(d)$ imaginary component. Results given for the leading-order solutions $(n=0$, solid) and firstorder corrections ( $n=1$, dashed).

wall was identified. The thickness of this boundary layer scales with the most probable distance travelled by a particle over a single oscillation period, $L_{c}$. For high oscillation frequencies, $\theta \gg 1$, this distance is much smaller than the mean free path $\lambda$ - reflected gas particles from the wall do not collide over this length scale, to leading order. This leads to dramatically different flow behaviour in comparison to the low frequency limit.

The leading-order mass distribution function of the gas in the bulk flow region (away from any walls) is directly proportional to the applied oscillatory body force, $a_{i}$. This immediately leads to a linearized Euler equation in that region. Higher-order corrections to the mass distribution function in $\theta^{-1}$ are proportional to gradients in the applied body force. Zero body force therefore yields no bulk flow.

The boundary layer near the wall, away from the bulk flow and termed the collisionless layer, was studied in detail. Solutions in this region were derived up to first order in $\theta^{-1}$. Interestingly, the complete set of boundary layer and bulk flow equations are independent of any differential or integral operators. This property yielded explicit and general solutions in all regions, which is unique to highly oscillatory and slightly rarefied flows.

Application of this general theory was illustrated for oscillatory thermal creep between two infinite and parallel walls. The absence of a body force in this model flow yields two localized flows at each wall - the related single-wall problem was therefore considered. The leading-order thermal creep flow, i.e. the mean tangential velocity component, was found to occur at first order $(n=1)$ in $\theta^{-1}$. The corresponding temperature, density and stress fields were also studied, and streamlines for the flow presented. 


\section{Acknowledgements}

The authors acknowledge financial support from an Australian Postgraduate Award and the Australian Research Council Grants Scheme.

\section{Supplementary data}

Supplementary data are available at http://dx.doi.org/10.1017/jfm.2013.281.

\section{Appendix A. Forms of the first-order corrections in the collisionless layer}

This appendix contains formulae for the first-order $(n=1)$ corrections within the collisionless layer. Expressions are given for the gas density $\sigma_{C}^{(1)}$, mean velocity $\bar{v}_{C \mid i}^{(1)}$, temperature $\tau_{C}^{(1)}$, and all components of the stress tensor $\epsilon_{C \mid i j}^{(1)}$; see (A 2)-(A 12).

All equations are specified in terms of the Kronecker delta function $\delta_{j k}$, the Abramowitz functions $J_{n}$ from (3.16), and $c_{i}^{(0)}$ defined in (3.15). Also, we define the integrals

$$
I_{m, n}\left(\eta_{C}\right)=\frac{1}{\pi} \begin{cases}\int_{0}^{\infty} J_{m}\left(-i y_{0}\right) J_{n}\left(-\mathrm{i}\left|\eta_{C}-y_{0}\right|\right) \mathrm{d} y_{0}, & n=2 s+1, \\ \int_{0}^{\infty} \operatorname{sgn}\left(\eta_{C}-y_{0}\right) J_{m}\left(-i y_{0}\right) J_{n}\left(-\mathrm{i}\left|\eta_{C}-y_{0}\right|\right) \mathrm{d} y_{0}, & n=2 s,\end{cases}
$$

where the indices $m, s \in \mathbb{Z}$. A full discussion of these functions and their numerical treatment is provided in appendix $\mathrm{C}$.

$$
\begin{aligned}
\sigma_{C}^{(1)}= & \left\{P_{b}^{(0)}\left(I_{1,0}\left(\eta_{C}\right)+\frac{1}{3}\left[I_{2,1}\left(\eta_{C}\right)-\frac{1}{2} I_{0,1}\left(\eta_{C}\right)-\frac{1}{2} I_{2,-1}\left(\eta_{C}\right)+\frac{7}{4} I_{0,-1}\left(\eta_{C}\right)\right]\right)\right. \\
& +2 c_{i}^{(0)} n_{i}\left(I_{2,0}\left(\eta_{C}\right)+\frac{1}{3}\left[I_{3,1}\left(\eta_{C}\right)-\frac{1}{2} I_{1,1}\left(\eta_{C}\right)-\frac{1}{2} I_{3,-1}\left(\eta_{C}\right)+\frac{7}{4} I_{1,-1}\left(\eta_{C}\right)\right]\right) \\
& +\tau_{b}^{(0)}\left(I_{3,0}\left(\eta_{C}\right)-\frac{3}{2} I_{1,0}\left(\eta_{C}\right)+\frac{1}{3}\left[I_{4,1}\left(\eta_{C}\right)-2 I_{2,1}\left(\eta_{C}\right)+\frac{7}{4} I_{0,1}\left(\eta_{C}\right)\right.\right. \\
& \left.\left.\left.-\frac{1}{2} I_{4,-1}\left(\eta_{C}\right)+\frac{5}{2} I_{2,-1}\left(\eta_{C}\right)-\frac{25}{8} I_{0,-1}\left(\eta_{C}\right)\right]\right)\right\} \\
& -\frac{\eta_{C}}{\sqrt{\pi}}\left[P_{b}^{(0)} J_{-1}\left(-\mathrm{i} \eta_{C}\right)+2 c_{i}^{(0)} n_{i} J_{0}\left(-\mathrm{i} \eta_{C}\right)+\tau_{b}^{(0)}\left(J_{1}\left(-\mathrm{i} \eta_{C}\right)-\frac{3}{2} J_{-1}\left(-\mathrm{i} \eta_{C}\right)\right)\right] \\
& +\frac{1}{\sqrt{\pi}}\left\{P_{b}^{(1)} J_{0}\left(-\mathrm{i} \eta_{C}\right)+2 V_{i}^{(1)} n_{i} J_{1}\left(-\mathrm{i} \eta_{C}\right)+\tau_{b}^{(1)}\left(J_{2}\left(-\mathrm{i} \eta_{C}\right)-\frac{3}{2} J_{0}\left(-\mathrm{i} \eta_{C}\right)\right)\right. \\
& \left.-\left.2 k \frac{\partial a_{j}}{\partial x_{k}}\right|_{\eta_{C}=0}\left(n_{k} n_{j}\left[J_{2}\left(-\mathrm{i} \eta_{C}\right)-\frac{1}{2} J_{0}\left(-\mathrm{i} \eta_{C}\right)\right]+\frac{\delta_{k j}}{2} J_{0}\left(-\mathrm{i} \eta_{C}\right)\right)\right\} \\
& -\frac{k \eta_{C}}{\sqrt{\pi}} J_{-1}\left(-\mathrm{i} \eta_{C}\right)\left[c_{i}^{(0)}\left(g_{2} t_{i}^{1}-g_{1} t_{i}^{2}+2 \bar{\kappa} n_{i}\right)+t_{j}^{1} \frac{\partial}{\partial x_{j}}\left(c_{i}^{(0)} t_{i}^{1}\right)+t_{j}^{2} \frac{\partial}{\partial x_{j}}\left(c_{i}^{(0)} t_{i}^{2}\right)\right] \\
& +\frac{\mathrm{i} k \eta_{C}^{2}}{2 \sqrt{\pi}}\left[P_{b}^{(0)} J_{-3}\left(-\mathrm{i} \eta_{C}\right)+2 c_{i}^{(0)} n_{i} J_{-2}\left(-\mathrm{i} \eta_{C}\right)+\tau_{b}^{(0)}\left(J_{-1}\left(-\mathrm{i} \eta_{C}\right)\right.\right. \\
& \left.\left.-\frac{1}{2} J_{-3}\left(-\mathrm{i} \eta_{C}\right)\right)\right], \\
& +2 c_{i}^{(0)} n_{i}\left(I_{2,1}\left(\eta_{C}\right)+\frac{1}{3}\left[I_{3,2}\left(\eta_{C}\right)-\frac{1}{2} I_{1,2}\left(\eta_{C}\right)-\frac{1}{2} I_{3,0}\left(\eta_{C}\right)+\frac{7}{4} I_{1,0}\left(\eta_{C}\right)\right]\right) \\
\bar{v}_{C \mid i}^{(1)} n_{i}=2 & P_{b}^{(0)}\left(I_{1,1}\left(\eta_{C}\right)+\frac{1}{3}\left[I_{2,2}\left(\eta_{C}\right)-\frac{1}{2} I_{0,2}\left(\eta_{C}\right)-\frac{1}{2} I_{2,0}\left(\eta_{C}\right)+\frac{7}{4} I_{0,0}\left(\eta_{C}\right)\right]\right) \\
&
\end{aligned}
$$




$$
\begin{aligned}
& +\tau_{b}^{(0)}\left(I_{3,1}\left(\eta_{C}\right)-\frac{3}{2} I_{1,1}\left(\eta_{C}\right)+\frac{1}{3}\left[I_{4,2}\left(\eta_{C}\right)-2 I_{2,2}\left(\eta_{C}\right)+\frac{7}{4} I_{0,2}\left(\eta_{C}\right)\right.\right. \\
& \left.\left.\left.-\frac{1}{2} I_{4,0}\left(\eta_{C}\right)+\frac{5}{2} I_{2,0}\left(\eta_{C}\right)-\frac{25}{8} I_{0,0}\left(\eta_{C}\right)\right]\right)\right\} \\
& -\frac{\eta_{C}}{\sqrt{\pi}}\left[P_{b}^{(0)} J_{0}\left(-\mathrm{i} \eta_{C}\right)+2 c_{i}^{(0)} n_{i} J_{1}\left(-\mathrm{i} \eta_{C}\right)+\tau_{b}^{(0)}\left(J_{2}\left(-\mathrm{i} \eta_{C}\right)-\frac{3}{2} J_{0}\left(-\mathrm{i} \eta_{C}\right)\right)\right] \\
& +\frac{1}{\sqrt{\pi}}\left\{P_{b}^{(1)} J_{1}\left(-\mathrm{i} \eta_{C}\right)+2 V_{i}^{(1)} n_{i} J_{2}\left(-\mathrm{i} \eta_{C}\right)+\tau_{b}^{(1)}\left(J_{3}\left(-\mathrm{i} \eta_{C}\right)-\frac{3}{2} J_{1}\left(-\mathrm{i} \eta_{C}\right)\right)\right. \\
& \left.-\left.2 k \frac{\partial a_{j}}{\partial x_{k}}\right|_{\eta_{C}=0}\left(n_{k} n_{j}\left[J_{3}\left(-\mathrm{i} \eta_{C}\right)-\frac{1}{2} J_{1}\left(-\mathrm{i} \eta_{C}\right)\right]+\frac{\delta_{k j}}{2} J_{1}\left(-\mathrm{i} \eta_{C}\right)\right)\right\} \\
& -\frac{k \eta_{C}}{\sqrt{\pi}} J_{0}\left(-\mathrm{i} \eta_{C}\right)\left[c_{i}^{(0)}\left(g_{2} t_{i}^{1}-g_{1} t_{i}^{2}+2 \bar{\kappa} n_{i}\right)+t_{j}^{1} \frac{\partial}{\partial x_{j}}\left(c_{i}^{(0)} t_{i}^{1}\right)+t_{j}^{2} \frac{\partial}{\partial x_{j}}\left(c_{i}^{(0)} t_{i}^{2}\right)\right] \\
& +\frac{\mathrm{i} k \eta_{C}^{2}}{2 \sqrt{\pi}} \bar{\kappa}\left[P_{b}^{(0)} J_{-2}\left(-\mathrm{i} \eta_{C}\right)+2 c_{i}^{(0)} n_{i} J_{-1}\left(-\mathrm{i} \eta_{C}\right)+\tau_{b}^{(0)}\left(J_{0}\left(-\mathrm{i} \eta_{C}\right)\right.\right. \\
& \left.\left.-\frac{1}{2} J_{-2}\left(-\mathrm{i} \eta_{C}\right)\right)\right] \\
& \bar{v}_{C \mid i}^{(1)} t_{i}^{1}=c_{i}^{(0)} t_{i}^{1}\left[I_{0,-1}\left(\eta_{C}\right)-\frac{\eta_{C}}{\sqrt{\pi}} J_{-1}\left(-\mathrm{i} \eta_{C}\right)\right]+\frac{1}{\sqrt{\pi}}\left[V_{i}^{(1)} t_{i}^{1} J_{0}\left(-\mathrm{i} \eta_{C}\right)\right. \\
& \left.-\left.k \frac{\partial a_{j}}{\partial x_{k}}\right|_{\eta_{C}=0}\left(t_{j}^{1} n_{k}+n_{j} t_{k}^{1}\right) J_{1}\left(-\mathrm{i} \eta_{C}\right)\right]-\frac{k \eta_{C}}{2 \sqrt{\pi}}\left[t_{j}^{1} \frac{\partial P_{b}^{(0)}}{\partial x_{j}} J_{-1}\left(-\mathrm{i} \eta_{C}\right)\right. \\
& +t_{j}^{1} \frac{\partial \tau_{b}^{(0)}}{\partial x_{j}}\left(J_{1}\left(-\mathrm{i} \eta_{C}\right)+\frac{1}{2} J_{-1}\left(-\mathrm{i} \eta_{C}\right)\right)-2 c_{i}^{(0)} \kappa_{i j} t_{j}^{1} J_{0}\left(-\mathrm{i} \eta_{C}\right) \\
& \left.+J_{0}\left(-\mathrm{i} \eta_{C}\right) t_{j}^{1} \frac{\partial}{\partial x_{j}}\left(2 c_{i}^{(0)} n_{i}\right)\right]+\frac{k \eta_{C}^{2}}{2 \sqrt{\pi}} J_{-3}\left(-\mathrm{i} \eta_{C}\right) c_{i}^{(0)}\left(\bar{\kappa} t_{i}^{1}+\kappa_{i j} t_{j}^{1}\right), \\
& \bar{v}_{C \mid i}^{(1)} t_{i}^{2}=c_{i}^{(0)} t_{i}^{2}\left[I_{0,-1}\left(\eta_{C}\right)-\frac{\eta_{C}}{\sqrt{\pi}} J_{-1}\left(-\mathrm{i} \eta_{C}\right)\right]+\frac{1}{\sqrt{\pi}}\left[V_{i}^{(1)} t_{i}^{2} J_{0}\left(-\mathrm{i} \eta_{C}\right)\right. \\
& \left.-\left.k \frac{\partial a_{j}}{\partial x_{k}}\right|_{\eta_{C}=0}\left(t_{j}^{2} n_{k}+n_{j} t_{k}^{2}\right) J_{1}\left(-\mathrm{i} \eta_{C}\right)\right]-\frac{k \eta_{C}}{2 \sqrt{\pi}}\left[t_{j}^{2} \frac{\partial P_{b}^{(0)}}{\partial x_{j}} J_{-1}\left(-\mathrm{i} \eta_{C}\right)\right. \\
& +t_{j}^{2} \frac{\partial \tau_{b}^{(0)}}{\partial x_{j}}\left(J_{1}\left(-\mathrm{i} \eta_{C}\right)+\frac{1}{2} J_{-1}\left(-\mathrm{i} \eta_{C}\right)\right)-2 c_{i}^{(0)} \kappa_{i j} t_{j}^{2} J_{0}\left(-\mathrm{i} \eta_{C}\right) \\
& \left.+J_{0}\left(-\mathrm{i} \eta_{C}\right) t_{j}^{2} \frac{\partial}{\partial x_{j}}\left(2 c_{i}^{(0)} n_{i}\right)\right]+\frac{k \eta_{C}^{2}}{2 \sqrt{\pi}} J_{-3}\left(-\mathrm{i} \eta_{C}\right) c_{i}^{(0)}\left(\bar{\kappa} t_{i}^{2}+\kappa_{i j} t_{j}^{2}\right), \\
& \frac{3}{2} \tau_{C}^{(1)}=2\left\{P _ { b } ^ { ( 0 ) } \left(I_{1,2}\left(\eta_{C}\right)-\frac{1}{2} I_{1,0}\left(\eta_{C}\right)+\frac{1}{3}\left[I_{2,3}\left(\eta_{C}\right)-\frac{1}{2} I_{0,3}\left(\eta_{C}\right)-I_{2,1}\left(\eta_{C}\right)\right.\right.\right. \\
& \left.\left.+2 I_{0,1}\left(\eta_{C}\right)+\frac{5}{4} I_{2,-1}\left(\eta_{C}\right)-\frac{11}{8} I_{0,-1}\left(\eta_{C}\right)\right]\right)+2 c_{i}^{(0)} n_{i}\left(I_{2,2}\left(\eta_{C}\right)\right. \\
& -\frac{1}{2} I_{2,0}\left(\eta_{C}\right)+\frac{1}{3}\left[I_{3,3}\left(\eta_{C}\right)-\frac{1}{2} I_{1,3}\left(\eta_{C}\right)-I_{3,1}\left(\eta_{C}\right)+2 I_{1,1}\left(\eta_{C}\right)\right. \\
& \left.\left.+\frac{5}{4} I_{3,-1}\left(\eta_{C}\right)-\frac{11}{8} I_{1,-1}\left(\eta_{C}\right)\right]\right)+\tau_{b}^{(0)}\left(I_{3,2}\left(\eta_{C}\right)-\frac{3}{2} I_{1,2}\left(\eta_{C}\right)-\frac{1}{2} I_{3,0}\left(\eta_{C}\right)\right. \\
& +\frac{3}{4} I_{1,0}\left(\eta_{C}\right)+\frac{1}{3}\left[I_{4,3}\left(\eta_{C}\right)-2 I_{2,3}\left(\eta_{C}\right)+\frac{7}{4} I_{0,3}\left(\eta_{C}\right)-I_{4,1}\left(\eta_{C}\right)\right. \\
& \left.\left.\left.+\frac{7}{2} I_{2,1}\left(\eta_{C}\right)-4 I_{0,1}\left(\eta_{C}\right)+\frac{5}{4} I_{4,-1}\left(\eta_{C}\right)-\frac{13}{4} I_{2,-1}\left(\eta_{C}\right)+\frac{53}{16} I_{0,-1}\left(\eta_{C}\right)\right]\right)\right\}
\end{aligned}
$$




$$
\begin{aligned}
& -\frac{\eta_{C}}{\sqrt{\pi}}\left[P_{b}^{(0)}\left(J_{1}\left(-\mathrm{i} \eta_{C}\right)-\frac{1}{2} J_{-1}\left(-\mathrm{i} \eta_{C}\right)\right)+2 c_{i}^{(0)} n_{i}\left(J_{2}\left(-\mathrm{i} \eta_{C}\right)-\frac{1}{2} J_{0}\left(-\mathrm{i} \eta_{C}\right)\right)\right. \\
& \left.+\tau_{b}^{(0)}\left(J_{3}\left(-\mathrm{i} \eta_{C}\right)-2 J_{1}\left(-\mathrm{i} \eta_{C}\right)+\frac{7}{4} J_{-1}\left(-\mathrm{i} \eta_{C}\right)\right)\right]+\frac{1}{\sqrt{\pi}}\left[P _ { b } ^ { ( 1 ) } \left(J_{2}\left(-\mathrm{i} \eta_{C}\right)\right.\right. \\
& \left.-\frac{1}{2} J_{0}\left(-\mathrm{i} \eta_{C}\right)\right)+2 V_{i}^{(1)} n_{i}\left(J_{3}\left(-\mathrm{i} \eta_{C}\right)-\frac{1}{2} J_{1}\left(-\mathrm{i} \eta_{C}\right)\right)+\tau_{b}^{(1)}\left(J_{4}\left(-\mathrm{i} \eta_{C}\right)\right. \\
& \left.-2 J_{2}\left(-\mathrm{i} \eta_{C}\right)+\frac{7}{4} J_{0}\left(-\mathrm{i} \eta_{C}\right)\right)-\left.2 k \frac{\partial a_{j}}{\partial x_{k}}\right|_{\eta_{C}=0}\left(n _ { k } n _ { j } \left[J_{4}\left(-\mathrm{i} \eta_{C}\right)-J_{2}\left(-\mathrm{i} \eta_{C}\right)\right.\right. \\
& \left.\left.\left.-\frac{1}{4} J_{0}\left(-\mathrm{i} \eta_{C}\right)\right]+\frac{\delta_{k j}}{2}\left[J_{2}\left(-\mathrm{i} \eta_{C}\right)+\frac{1}{2} J_{0}\left(-\mathrm{i} \eta_{C}\right)\right]\right)\right]-\frac{k \eta_{C}}{\sqrt{\pi}}\left(J_{1}\left(-\mathrm{i} \eta_{C}\right)\right. \\
& \left.+\frac{1}{2} J_{-1}\left(-\mathrm{i} \eta_{C}\right)\right)\left[c_{i}^{(0)}\left(g_{2} t_{i}^{1}-g_{1} t_{i}^{2}+2 \bar{\kappa} n_{i}\right)+t_{j}^{1} \frac{\partial}{\partial x_{j}}\left(c_{i}^{(0)} t_{i}^{1}\right)\right. \\
& \left.+t_{j}^{2} \frac{\partial}{\partial x_{j}}\left(c_{i}^{(0)} t_{i}^{2}\right)\right]+\frac{\mathrm{i} k \eta_{C}^{2}}{2 \sqrt{\pi}} \bar{\kappa}\left[P_{b}^{(0)}\left(J_{-1}\left(-\mathrm{i} \eta_{C}\right)+\frac{1}{2} J_{-3}\left(-\mathrm{i} \eta_{C}\right)\right)\right. \\
& \left.+2 c_{i}^{(0)} n_{i}\left(J_{0}\left(-\mathrm{i} \eta_{C}\right)+\frac{1}{2} J_{-2}\left(-\mathrm{i} \eta_{C}\right)\right)+\tau_{b}^{(0)}\left(J_{1}\left(-\mathrm{i} \eta_{C}\right)+\frac{7}{4} J_{-3}\left(-\mathrm{i} \eta_{C}\right)\right)\right], \\
& \frac{1}{2} \epsilon_{C \mid i j}^{(1)} n_{i} n_{j}=2\left\{P_{b}^{(0)}\left(I_{1,2}\left(\eta_{C}\right)+\frac{1}{3}\left[I_{2,3}\left(\eta_{C}\right)-\frac{1}{2} I_{0,3}\left(\eta_{C}\right)-\frac{1}{2} I_{2,1}\left(\eta_{C}\right)+\frac{7}{4} I_{0,1}\left(\eta_{C}\right)\right]\right)\right. \\
& +2 c_{i}^{(0)} n_{i}\left(I_{2,2}\left(\eta_{C}\right)+\frac{1}{3}\left[I_{3,3}\left(\eta_{C}\right)-\frac{1}{2} I_{1,3}\left(\eta_{C}\right)-\frac{1}{2} I_{3,1}\left(\eta_{C}\right)\right.\right. \\
& \left.\left.+\frac{7}{4} I_{1,1}\left(\eta_{C}\right)\right]\right)+\tau_{b}^{(0)}\left(I_{3,2}\left(\eta_{C}\right)-\frac{3}{2} I_{1,2}\left(\eta_{C}\right)+\frac{1}{3}\left[I_{4,3}\left(\eta_{C}\right)-2 I_{2,3}\left(\eta_{C}\right)\right.\right. \\
& \left.\left.\left.+\frac{7}{4} I_{0,3}\left(\eta_{C}\right)-\frac{1}{2} I_{4,1}\left(\eta_{C}\right)+\frac{5}{2} I_{2,1}\left(\eta_{C}\right)-\frac{25}{8} I_{0,1}\left(\eta_{C}\right)\right]\right)\right\} \\
& -\frac{\eta_{C}}{\sqrt{\pi}}\left[P_{b}^{(0)} J_{1}\left(-\mathrm{i} \eta_{C}\right)+2 c_{i}^{(0)} n_{i} J_{2}\left(-\mathrm{i} \eta_{C}\right)+\tau_{b}^{(0)}\left(J_{3}\left(-\mathrm{i} \eta_{C}\right)\right.\right. \\
& \left.\left.-\frac{3}{2} J_{1}\left(-\mathrm{i} \eta_{C}\right)\right)\right]+\frac{1}{\sqrt{\pi}}\left[P_{b}^{(1)} J_{2}\left(-\mathrm{i} \eta_{C}\right)+2 V_{i}^{(1)} n_{i} J_{3}\left(-\mathrm{i} \eta_{C}\right)+\tau_{b}^{(1)}\left(J_{4}\left(-\mathrm{i} \eta_{C}\right)\right.\right. \\
& \left.-\frac{3}{2} J_{2}\left(-\mathrm{i} \eta_{C}\right)\right)-\left.2 k \frac{\partial a_{j}}{\partial x_{k}}\right|_{\eta_{C}=0}\left(n_{k} n_{j}\left[J_{4}\left(-\mathrm{i} \eta_{C}\right)-\frac{1}{2} J_{2}\left(-\mathrm{i} \eta_{C}\right)\right]\right. \\
& \left.\left.+\frac{\delta_{k j}}{2} J_{2}\left(-\mathrm{i} \eta_{C}\right)\right)\right]-\frac{k \eta_{C}}{\sqrt{\pi}} J_{1}\left(-\mathrm{i} \eta_{C}\right)\left[c_{i}^{(0)}\left(g_{2} t_{i}^{1}-g_{1} t_{i}^{2}+2 \bar{\kappa} n_{i}\right)\right. \\
& \left.+t_{j}^{1} \frac{\partial}{\partial x_{j}}\left(c_{i}^{(0)} t_{i}^{1}\right)+t_{j}^{2} \frac{\partial}{\partial x_{j}}\left(c_{i}^{(0)} t_{i}^{2}\right)\right]+\frac{\mathrm{i} k \eta_{C}^{2}}{2 \sqrt{\pi}} \bar{\kappa}\left[P_{b}^{(0)} J_{-1}\left(-\mathrm{i} \eta_{C}\right)\right. \\
& \left.+2 c_{i}^{(0)} n_{i} J_{0}\left(-\mathrm{i} \eta_{C}\right)+\tau_{b}^{(0)}\left(J_{1}\left(-\mathrm{i} \eta_{C}\right)-\frac{1}{2} J_{-1}\left(-\mathrm{i} \eta_{C}\right)\right)\right], \\
& \epsilon_{C \mid i j}^{(1)} t_{i}^{1} t_{j}^{1}=2\left\{P_{b}^{(0)}\left(I_{1,0}\left(\eta_{C}\right)+\frac{1}{3}\left[I_{2,1}\left(\eta_{C}\right)-\frac{1}{2} I_{0,1}\left(\eta_{C}\right)+\frac{1}{2} I_{2,-1}\left(\eta_{C}\right)+\frac{5}{4} I_{0,-1}\left(\eta_{C}\right)\right]\right)\right. \\
& +2 c_{i}^{(0)} n_{i}\left(I_{2,0}\left(\eta_{C}\right)+\frac{1}{3}\left[I_{3,1}\left(\eta_{C}\right)-\frac{1}{2} I_{1,1}\left(\eta_{C}\right)+\frac{1}{2} I_{3,-1}\left(\eta_{C}\right)\right.\right. \\
& \left.\left.+\frac{5}{4} I_{1,-1}\left(\eta_{C}\right)\right]\right)+\tau_{b}^{(0)}\left(I_{3,0}\left(\eta_{C}\right)-\frac{3}{2} I_{1,0}\left(\eta_{C}\right)+\frac{1}{3}\left[I_{4,1}\left(\eta_{C}\right)-2 I_{2,1}\left(\eta_{C}\right)\right.\right. \\
& \left.\left.\left.+\frac{7}{4} I_{0,1}\left(\eta_{C}\right)+\frac{1}{2} I_{4,-1}\left(\eta_{C}\right)+\frac{1}{2} I_{2,-1}\left(\eta_{C}\right)-\frac{11}{8} I_{0,-1}\left(\eta_{C}\right)\right]\right)\right\} \\
& -\frac{\eta_{C}}{\sqrt{\pi}}\left[P_{b}^{(0)} J_{-1}\left(-\mathrm{i} \eta_{C}\right)+2 c_{i}^{(0)} n_{i} J_{0}\left(-\mathrm{i} \eta_{C}\right)+\tau_{b}^{(0)}\left(J_{1}\left(-\mathrm{i} \eta_{C}\right)-\frac{1}{2} J_{-1}\left(-\mathrm{i} \eta_{C}\right)\right)\right]
\end{aligned}
$$




$$
\begin{aligned}
& +\frac{1}{\sqrt{\pi}}\left[P_{b}^{(1)} J_{0}\left(-\mathrm{i} \eta_{C}\right)+2 V_{i}^{(1)} n_{i} J_{1}\left(-\mathrm{i} \eta_{C}\right)+\tau_{b}^{(1)}\left(J_{2}\left(-\mathrm{i} \eta_{C}\right)\right.\right. \\
& \left.-\frac{1}{2} J_{0}\left(-\mathrm{i} \eta_{C}\right)\right)-\left.k \frac{\partial a_{j}}{\partial x_{k}}\right|_{\eta_{C}=0}\left(n_{k} n_{j}\left[J_{2}\left(-\mathrm{i} \eta_{C}\right)-\frac{1}{2} J_{0}\left(-\mathrm{i} \eta_{C}\right)\right]\right. \\
& \left.+\frac{\delta_{k j}}{2} J_{0}\left(-\mathrm{i} \eta_{C}\right)\right)-k\left(t_{k}^{1} \frac{\left.\partial\left(a_{j} t_{j}^{1}\right)\right|_{\eta_{C}=0}}{\partial x_{k}}+\left.\kappa_{1}\left(a_{j} n_{j}\right)\right|_{\eta_{C}=0}-\left.g_{1}\left(a_{j} t_{j}^{2}\right)\right|_{\eta_{C}=0}\right) \\
& \left.\times J_{0}\left(-\mathrm{i} \eta_{C}\right)\right]-\frac{k \eta_{C}}{\sqrt{\pi}} J_{-1}\left(-\mathrm{i} \eta_{C}\right)\left[c_{i}^{(0)}\left(g_{2} t_{i}^{1}-3 g_{1} t_{i}^{2}+\left[3 \kappa_{1}+\kappa_{2}\right] n_{i}\right)\right. \\
& \left.+3 t_{j}^{1} \frac{\partial}{\partial x_{j}}\left(c_{i}^{(0)} t_{i}^{1}\right)+t_{j}^{2} \frac{\partial}{\partial x_{j}}\left(c_{i}^{(0)} t_{i}^{2}\right)\right]+\frac{\mathrm{i} k \eta_{C}^{2}}{4 \sqrt{\pi}}\left(3 \kappa_{1}+\kappa_{2}\right)\left[P_{b}^{(0)} J_{-3}\left(-\mathrm{i} \eta_{C}\right)\right. \\
& \left.+2 c_{i}^{(0)} n_{i} J_{-2}\left(-\mathrm{i} \eta_{C}\right)+\tau_{b}^{(0)}\left(J_{-1}\left(-\mathrm{i} \eta_{C}\right)-\frac{1}{2} J_{-3}\left(-\mathrm{i} \eta_{C}\right)\right)\right], \\
& \epsilon_{C|| i j}^{(1)} t_{i}^{2} t_{j}^{2}=2\left\{P_{b}^{(0)}\left(I_{1,0}\left(\eta_{C}\right)+\frac{1}{3}\left[I_{2,1}\left(\eta_{C}\right)-\frac{1}{2} I_{0,1}\left(\eta_{C}\right)+\frac{1}{2} I_{2,-1}\left(\eta_{C}\right)+\frac{5}{4} I_{0,-1}\left(\eta_{C}\right)\right]\right)\right. \\
& +2 c_{i}^{(0)} n_{i}\left(I_{2,0}\left(\eta_{C}\right)+\frac{1}{3}\left[I_{3,1}\left(\eta_{C}\right)-\frac{1}{2} I_{1,1}\left(\eta_{C}\right)+\frac{1}{2} I_{3,-1}\left(\eta_{C}\right)\right.\right. \\
& \left.\left.+\frac{5}{4} I_{1,-1}\left(\eta_{C}\right)\right]\right)+\tau_{b}^{(0)}\left(I_{3,0}\left(\eta_{C}\right)-\frac{3}{2} I_{1,0}\left(\eta_{C}\right)+\frac{1}{3}\left[I_{4,1}\left(\eta_{C}\right)-2 I_{2,1}\left(\eta_{C}\right)\right.\right. \\
& \left.\left.\left.+\frac{7}{4} I_{0,1}\left(\eta_{C}\right)+\frac{1}{2} I_{4,-1}\left(\eta_{C}\right)+\frac{1}{2} I_{2,-1}\left(\eta_{C}\right)-\frac{11}{8} I_{0,-1}\left(\eta_{C}\right)\right]\right)\right\} \\
& -\frac{\eta_{C}}{\sqrt{\pi}}\left[P_{b}^{(0)} J_{-1}\left(-\mathrm{i} \eta_{C}\right)+2 c_{i}^{(0)} n_{i} J_{0}\left(-\mathrm{i} \eta_{C}\right)+\tau_{b}^{(0)}\left(J_{1}\left(-\mathrm{i} \eta_{C}\right)\right.\right. \\
& \left.\left.-\frac{1}{2} J_{-1}\left(-\mathrm{i} \eta_{C}\right)\right)\right]+\frac{1}{\sqrt{\pi}}\left[P_{b}^{(1)} J_{0}\left(-\mathrm{i} \eta_{C}\right)+2 V_{i}^{(1)} n_{i} J_{1}\left(-\mathrm{i} \eta_{C}\right)+\tau_{b}^{(1)}\left(J_{2}\left(-\mathrm{i} \eta_{C}\right)\right.\right. \\
& \left.-\frac{1}{2} J_{0}\left(-\mathrm{i} \eta_{C}\right)\right)-\left.k \frac{\partial a_{j}}{\partial x_{k}}\right|_{\eta_{C}=0}\left(n_{k} n_{j}\left[J_{2}\left(-\mathrm{i} \eta_{C}\right)-\frac{1}{2} J_{0}\left(-\mathrm{i} \eta_{C}\right)\right]\right. \\
& \left.+\frac{\delta_{k j}}{2} J_{0}\left(-\mathrm{i} \eta_{C}\right)\right)-k\left(t_{k}^{2} \frac{\left.\partial\left(a_{j} t_{j}^{2}\right)\right|_{\eta_{C}=0}}{\partial x_{k}}+\left.\kappa_{2}\left(a_{j} n_{j}\right)\right|_{\eta_{C}=0}+\left.g_{2}\left(a_{j} t_{j}^{1}\right)\right|_{\eta_{C}=0}\right) \\
& \left.\times J_{0}\left(-\mathrm{i} \eta_{C}\right)\right]-\frac{k \eta_{C}}{\sqrt{\pi}} J_{-1}\left(-\mathrm{i} \eta_{C}\right)\left[c_{i}^{(0)}\left(3 g_{2} t_{i}^{1}-g_{1} t_{i}^{2}+\left[\kappa_{1}+3 \kappa_{2}\right] n_{i}\right)\right. \\
& \left.+t_{j}^{1} \frac{\partial}{\partial x_{j}}\left(c_{i}^{(0)} t_{i}^{1}\right)+3 t_{j}^{2} \frac{\partial}{\partial x_{j}}\left(c_{i}^{(0)} t_{i}^{2}\right)\right] \\
& +\frac{\mathrm{i} k \eta_{C}^{2}}{4 \sqrt{\pi}}\left(\kappa_{1}+3 \kappa_{2}\right)\left[P_{b}^{(0)} J_{-3}\left(-\mathrm{i} \eta_{C}\right)+2 c_{i}^{(0)} n_{i} J_{-2}\left(-\mathrm{i} \eta_{C}\right)\right. \\
& \left.+\tau_{b}^{(0)}\left(J_{-1}\left(-\mathrm{i} \eta_{C}\right)-\frac{1}{2} J_{-3}\left(-\mathrm{i} \eta_{C}\right)\right)\right] \\
& \frac{1}{2} \epsilon_{C \mid i j}^{(1)} n_{i} t_{j}^{1}=c_{i}^{(0)} t_{i}^{1}\left[I_{0,0}\left(\eta_{C}\right)-\frac{\eta_{C}}{\sqrt{\pi}} J_{0}\left(-\mathrm{i} \eta_{C}\right)\right]+\frac{1}{\sqrt{\pi}}\left[V_{i}^{(1)} t_{i}^{1} J_{1}\left(-\mathrm{i} \eta_{C}\right)\right. \\
& \left.-\left.k \frac{\partial a_{j}}{\partial x_{k}}\right|_{\eta_{C}=0}\left(t_{j}^{1} n_{k}+n_{j} t_{k}^{1}\right) J_{2}\left(-\mathrm{i} \eta_{C}\right)\right]-\frac{k \eta_{C}}{2 \sqrt{\pi}}\left[t_{j}^{1} \frac{\partial P_{b}^{(0)}}{\partial x_{j}} J_{0}\left(-\mathrm{i} \eta_{C}\right)\right. \\
& +t_{j}^{1} \frac{\partial \tau_{b}^{(0)}}{\partial x_{j}}\left(J_{2}\left(-\mathrm{i} \eta_{C}\right)+\frac{1}{2} J_{0}\left(-\mathrm{i} \eta_{C}\right)\right)-2 c_{i}^{(0)} \kappa_{i j} t_{j}^{1} J_{1}\left(-\mathrm{i} \eta_{C}\right)
\end{aligned}
$$




$$
\begin{aligned}
& \left.+J_{1}\left(-\mathrm{i} \eta_{C}\right) t_{j}^{1} \frac{\partial}{\partial x_{j}}\left(2 c_{i}^{(0)} n_{i}\right)\right]+\frac{k \eta_{C}^{2}}{2 \sqrt{\pi}} J_{-2}\left(-\mathrm{i} \eta_{C}\right) c_{i}^{(0)}\left(\bar{\kappa} t_{i}^{1}+\kappa_{i j} t_{j}^{1}\right) \\
= & \frac{1}{2} \epsilon_{C \mid j i}^{(1)} n_{i} t_{j}^{1}, \\
\frac{1}{2} \epsilon_{C \mid j i j}^{(1)} n_{i} t_{j}^{2}= & t_{i}^{2} c_{i}^{(0)}\left[I_{0,0}\left(\eta_{C}\right)-\frac{\eta_{C}}{\sqrt{\pi}} J_{0}\left(-\mathrm{i} \eta_{C}\right)\right]+\frac{1}{\sqrt{\pi}}\left[V_{i}^{(1)} t_{i}^{2} J_{1}\left(-\mathrm{i} \eta_{C}\right)\right. \\
& \left.-\left.k \frac{\partial a_{j}}{\partial x_{k}}\right|_{\eta_{C}=0}\left(t_{j}^{2} n_{k}+n_{j} t_{k}^{2}\right) J_{2}\left(-\mathrm{i} \eta_{C}\right)\right]-\frac{k \eta_{C}}{2 \sqrt{\pi}}\left[t_{j}^{2} \frac{\partial P_{b}^{(0)}}{\partial x_{j}} J_{0}\left(-\mathrm{i} \eta_{C}\right)\right. \\
& +t_{j}^{2} \frac{\partial \tau_{b}^{(0)}}{\partial x_{j}}\left(J_{2}\left(-\mathrm{i} \eta_{C}\right)+\frac{1}{2} J_{0}\left(-\mathrm{i} \eta_{C}\right)\right)-2 c_{i}^{(0)} \kappa_{i j} t_{j}^{2} J_{1}\left(-\mathrm{i} \eta_{C}\right) \\
& \left.+J_{1}\left(-\mathrm{i} \eta_{C}\right) t_{j}^{2} \frac{\partial}{\partial x_{j}}\left(2 c_{i}^{(0)} n_{i}\right)\right]+\frac{k \eta_{C}^{2}}{2 \sqrt{\pi}} J_{-2}\left(-\mathrm{i} \eta_{C}\right) c_{i}^{(0)}\left(\bar{\kappa} t_{i}^{2}+\kappa_{i j} t_{j}^{2}\right) \\
= & \frac{1}{2} \epsilon_{C \mid j i}^{(1)} n_{i} t_{j}^{2}, \\
\epsilon_{C \mid i j}^{(1)} t_{i}^{1} t_{j}^{2}= & -\left.\frac{k}{2 \sqrt{\pi}} \frac{\partial a_{j}}{\partial x_{k}}\right|_{\eta_{C}=0}\left(t_{j}^{1} t_{k}^{2}+t_{k}^{1} t_{j}^{2}\right) J_{0}\left(-\mathrm{i} \eta_{C}\right)-\frac{k \eta_{C}}{\sqrt{\pi}} J_{-1}\left(-\mathrm{i} \eta_{C}\right) \\
& \times\left[c_{i}^{(0)}\left(g_{1} t_{i}^{1}-g_{2} t_{i}^{2}\right)+t_{i}^{2} \frac{\partial}{\partial x_{i}}\left(c_{j}^{(0)} t_{j}^{1}\right)+t_{i}^{1} \frac{\partial}{\partial x_{i}}\left(c_{j}^{(0)} t_{j}^{2}\right)\right] \\
= & \epsilon_{C \mid j i}^{(1)} t_{i}^{1} t_{j}^{2} .
\end{aligned}
$$

Appendix B. Proof by induction that $\phi_{K}^{(n)} \equiv 0$ for all $n \geqslant 0$

In this appendix, we provide a proof by induction of the following proposition.

PROPOSITION 1. The $n$ th-order components of the $\theta^{-1}$-expansion of the mass distribution function within the Knudsen layer, represented by $\phi_{K}^{(n)}$, are uniquely zero for all $n \geqslant 0$.

Proof. Let $P_{n}$ be the proposition that the mass distribution within the Knudsen layer $\phi_{K}^{(n)}$ at order $n$ is zero.

From (3.7), $P_{0}$ is true. Substituting this into the moment equations in (3.2), we find the corresponding density $\sigma_{K}^{(0)}$, mean velocity $\bar{v}_{K \mid i}^{(0)}$, temperature $\tau_{K}^{(0)}$ and stress $\epsilon_{K \mid i j}^{(0)}$ are also zero.

Setting $n=1$ in the second line of (3.6), the right-hand side reduces to zero. Thus, $P_{0}$ implies $P_{1}$.

Finally, we assume $P_{m}$ is true, i.e. $\phi_{K}^{(m)}=0$. All moments of $\phi_{K}^{(m)}$ in (3.2) are also zero. Setting $n=m+1$ in the second line of (3.6), it is evident that $P_{m}$ implies $P_{m+1}$, i.e. $\phi_{K}^{(m)}=0$ implies that $\phi_{K}^{(m+1)}=0$.

To conclude, $P_{0}$ is true. This implies $P_{1}$ is also true; assuming $P_{m}$ to be true for $m \geqslant 1$, we have shown $P_{m+1}$ to be true. Therefore, by induction we conclude that $P_{n}$ is true for all integer $n \geqslant 0$, and $\phi_{K}^{(n)}=0$.

\section{Appendix C. The Abramowitz functions}

We now discuss the Abramowitz functions defined in (3.16). For a pure real $\eta$, the Abramowitz functions can be expressed in terms of the in-built Meijer-G function 
in MATHEMATICA ${ }^{\circledR}$ (see (C 1) and Loyalka \& Tompson 2009):

$$
J_{n}(\eta)=\frac{\eta^{n+1}}{2^{n+2} \sqrt{\pi}} \text { MeijerG }\left(\{\{\},\{\}\},\left\{\left\{0,-\frac{n+1}{2},-\frac{n}{2}\right\},\{\}\right\}, \frac{\eta^{2}}{4}\right) .
$$

This representation is accurate for all $\eta \neq 0$; at $\eta=0$ the Abramowitz functions reduce to Gaussian moment integrals, which are easily evaluated analytically.

In this article, $\eta$ is purely complex - expressed in plane polar form, the argument of this complex number is $-\pi / 2$; see table 2 and appendix A. The correct expression for the Abramowitz functions in this case utilizes the in-built Conjugate function in MATHEMATICA $^{\circledR}$, and is

$$
\begin{aligned}
J_{n}(-\mathrm{i} \eta)= & \text { Conjugate }\left[\frac{(\mathrm{i} \eta)^{n+1}}{2^{n+2} \sqrt{\pi}}\right. \\
& \left.\times \text { MeijerG }\left(\{\{\},\{\}\},\left\{\left\{0,-\frac{n+1}{2},-\frac{n}{2}\right\},\{\}\right\}, \frac{(\mathrm{i} \eta)^{2}}{4}\right)\right] .
\end{aligned}
$$

All numerical results in this article utilize (C2) to evaluate the Abramowitz functions. Also, for large modulus $\eta$, the Abramowitz functions decay rapidly to zero.

As discussed in appendix A, the first-order corrections to the flow within the collisionless layer are defined in terms of the $I_{m, n}\left(\eta_{C}\right)$ integrals; see (A 1). These integrals take the variable $\eta_{C}$; in this article, $\eta_{C}$ is the scaled distance in the normal direction from a wall positioned at $\eta_{C}=0$. The length scale is $L_{c}$, which is the thickness of the collisionless layer; see figure 1. The integrand of $I_{m, n}\left(\eta_{C}\right)$ is composed of a product of Abramowitz functions of the general form given in (C2) - from before, the $J_{n}(-\mathrm{i} \eta)$ functions are highly oscillatory in $\eta$. Hence, the integrals $I_{m, n}\left(\eta_{C}\right)$ from (A 1) are computationally expensive to evaluate. To facilitate application of the theory in this article, the complete set of the $I_{m, n}\left(\eta_{C}\right)$ integrals necessary to study the flow using the formulae in appendix A were evaluated numerically. The supplementary material to this article available at http://dx.doi.org/10.1017/jfm.2013.281 includes data sets for $I_{m, n}\left(\eta_{C}\right)$, spanning all pairs of $(m, n)$ for the range $m=0-4$, and $n=-1$ to 3 .

We now discuss the numerical technique applied to construct the data sets. The integrals for $n=-1$ to 1 were evaluated by direct numerical integration of (A 1 ) using the in-built MATHEMATICA ${ }^{\circledR}$ function NIntegrate, over the domain $\eta_{C} \in[0,16]$ with a step size of 0.01 . This domain extends a distance of $16 L_{c}$ from the wall, and provides sufficient scope to study the flow within the collisionless layer near the wall, which is of thickness $L_{c}$; see figure 1. To evaluate the $I_{m, n}\left(\eta_{C}\right)$ integrals numerically, the integration region was truncated to $y_{0} \in[0,50]$. The upper limit $\left(y_{0}=50\right)$ yields a sufficiently small integrand, which for $y_{0} \geqslant 50$ is zero to at least eight decimal places for all $(m, n)$. Finally, the relative and absolute tolerances were each set to $10^{-5}$ using the AccuracyGoal and PrecisionGoal functions in MATHEMATICA ${ }^{\circledR}$.

The numerical precision of this approach was explored using convergence studies. At the wall, i.e. at $\eta_{C}=0$, an upper bound of $10^{-5}$ was established for the error magnitude of $I_{m, n}(0)$; this finding is independent of $m$ and $n$. In contrast, the maximum error magnitude of $I_{m, n}\left(\eta_{C}\right)$ is $10^{-3}$ for $\eta_{C}>0$; this is because the collisionless boundary layer solutions are localized at the wall and exhibit decay for increasing $\eta_{C}$, while the absolute and relative tolerances remain fixed. The numerical errors compound when the $I_{m, n}\left(\eta_{C}\right)$ numerical data is applied to study of the $O\left(\theta^{-1}\right)$ collisionless layer hydrodynamic quantities from appendix A. In non-dimensional units, this results in solutions that exhibit a maximum error magnitude of (i) $10^{-4}$ at the wall, and (ii) $10^{-2}$ away from the wall. 
To reduce the computational time required to evaluate the higher-order integrals with $n=2$ and $n=3$, we first substitute for $J_{n}\left(-\mathrm{i}\left|\eta_{C}-y_{0}\right|\right)$ in the integrand of (A 1) using the recurrence relation (Abramowitz \& Stegun 1965):

$$
J_{s+3}(x)=\frac{1}{2}\left(x J_{s}(x)+(s+2) J_{s+1}(x)\right),
$$

where the index $s \in \mathbb{Z}$. Equation (C3) can be derived using integration by parts. Substituting (C 3) with $s=-1$ into (A 1) for $n=2$ gives

$$
\begin{aligned}
I_{m, 2}\left(\eta_{C}\right)= & \frac{1}{\pi} \int_{0}^{\infty}\left(-\mathrm{i}\left|\eta_{C}-y_{0}\right|\right) \operatorname{sgn}\left(\eta_{C}-y_{0}\right) J_{m}\left(-i y_{0}\right) J_{-1}\left(-\mathrm{i}\left|\eta_{C}-y_{0}\right|\right) \mathrm{d} y_{0} \\
& +I_{m, 0}\left(\eta_{C}\right) .
\end{aligned}
$$

The integral $I_{m, 0}\left(\eta_{C}\right)$ is already required, and is easily evaluated using the approach outlined. Also, the remaining term on the right-hand side of (C4) is more amenable to numerical integration using NIntegrate, thus reducing computation time. Similar arguments for $s=0$ and $n=3$ yield the expression

$$
I_{m, 3}\left(\eta_{C}\right)=\frac{1}{\pi} \int_{0}^{\infty}\left(-\mathrm{i}\left|\eta_{C}-y_{0}\right|\right) J_{m}\left(-\mathrm{i} y_{0}\right) J_{0}\left(-\mathrm{i}\left|\eta_{C}-y_{0}\right|\right) \mathrm{d} y_{0}+2 I_{m, 1}\left(\eta_{C}\right),
$$

where once more, integration of the former of the two expressions using NIntegrate is more efficient than directly integrating $I_{m, 3}\left(\eta_{C}\right)$, and $I_{m, 1}\left(\eta_{C}\right)$ is already required.

The full numerical data set in the serialized package integralData.mx can be accessed by running the code in the supplementary MATHEMATICA ${ }^{\circledR}$ notebook integralDataImporter.nb. For further information regarding the syntax, see the notebook file.

\section{REFERENCES}

Abramowitz, M. \& Stegun, I. A. 1965 Handbook of Mathematical Functions. Dover.

Agarwal, R. K., Yun, K. Y. \& Balakrishnan, R. 2001 Beyond Navier-Stokes: Burnett equations for flows in the continuum-transition regime. Phys. Fluids 13 (10), 3061.

BAKer, L. L. \& Hadjiconstantinou, N. G. 2005 Variance reduction for Monte Carlo solutions of the Boltzmann equation. Phys. Fluids 17 (5), 051703.

Bargatin, I., Kozinsky, I. \& Roukes, M. L. 2007 Efficient electrothermal actuation of multiple modes of high-frequency nanoelectromechanical resonators. Appl. Phys. Lett. 90 (9), 093116.

Bhatnagar, P. L., Gross, E. P. \& Krook, M. 1954 A model for collision processes in gases. Part 1. Small amplitude processes in charged and neutral one-component systems. Phys. Rev. 94 (3), 511.

BIRD, G. A. 1963 Approach to translational equilibrium in a rigid sphere gas. Phys. Fluids 6, 1518-1519.

BIRD, G. A. 1998 Recent advances and current challenges for DSMC. Comput. Maths Applics. 35 (1-2), 1-14.

Bittencourt, J. A. 2004 Fundamentals of Plasma Physics. Springer.

Bouchut, F. \& Perthame, B. 1993 A BGK model for small Prandtl number in the Navier-Stokes approximation. J. Stat. Phys. 71 (1-2), 191-207.

BRUll, S. \& SCHNEIDER, J. 2008 A new approach for the ellipsoidal statistical model. Contin. Mech. Thermodyn. 20 (2), 63-74.

Camenzind, M. 2007 Compact Objects in Astrophysics: White Dwarfs, Neutron Stars, and Black Holes. Springer.

Cartan, H. 1977 Course de Calcul Différentiel. Hermann.

CERCIGNANI, C. 1962 Elementary solutions of the linearized gas-dynamics Boltzmann equation and their application to the slip-flow problem. Ann. Phys. 20 (2), 219-233. 
Cercignani, C. 1964 Higher order slip according to the linearized Boltzmann equation. Tech. Rep., California University Berkeley Institute of Engineering Research.

Cercignani, C. 1988 The Boltzmann Equation and its Applications. Springer.

Cercignani, C. 2000 Rarefied Gas Dynamics: From Basic Concepts to Actual Calculations. Cambridge University Press.

ChAPMAN, S. 1916 On the law of distribution of molecular velocities, and on the theory of viscosity and thermal conduction, in a non-uniform simple monatomic gas. Phil. Trans. R. Soc. Lond. A 216, 279-348.

Chen, H., Kandasamy, S., Orszag, S., Shock, R., Succi, S. \& Yakhot, V. 2003 Extended Boltzmann kinetic equation for turbulent flows. Science 301 (5633), 633-636.

Chun, J. \& Koch, D. L. 2005 A direct simulation Monte Carlo method for rarefied gas flows in the limit of small Mach number. Phys. Fluids 17 (10), 107107.

Clausing, P. 1971 The flow of highly rarefied gases through tubes of arbitrary length. J. Vac. Sci. Technol. 8 (5), 636-646.

Dodelson, S. 2003 Modern Cosmology. Academic.

Dubois, J.-M., Ouanounou, G. \& Rouzaire-Dubois, B. 2009 The Boltzmann equation in molecular biology. Prog. Biophys. Molecular Biol. 99 (2-3), 87-93.

Enskog, D. 1917 Kinetische Theorie der Vorgänge in mässig verdünnten Gasen. PhD thesis, Uppsala.

GALlis, M. A. \& TORCZYNSKI, J. R. 2011 Investigation of the ellipsoidal-statistical Bhatnagar-Gross-Krook kinetic model applied to gas-phase transport of heat and tangential momentum between parallel walls. Phys. Fluids 23 (3), 030601.

GrAD, H. 1958 Principles of the Kinetic Theory of Gases. Springer.

HADJiconstantinou, N. G. $2005 a$ Oscillatory shear-driven gas flows in the transition and free-molecular-flow regimes. Phys. Fluids 17 (10), 100611.

Hadjiconstantinou, N. G. $2005 b$ Validation of a second-order slip model for dilute gas flows. Microscale Therm. Engng 9 (2), 137-153.

Hadjiconstantinou, N. G., Garcia, A. L., Bazant, M. Z. \& He, G. 2003 Statistical error in particle simulations of hydrodynamic phenomena. J. Comput. Phys. 187 (1), 274-297.

Hadjiconstantinou, N. G., Radtke, G. A. \& Baker, L. L. 2010 On variance-reduced simulations of the Boltzmann transport equation for small-scale heat transfer applications. J. Heat Transfer 132 (11), 112401.

Hilbert, D. 1900 Mathematische Probleme. In Vortrag, Gehalten auf dem Internationalen Mathematiker, pp. 253-297. Vadenhöck and Ruprecht.

HILberT, D. 1912 Grundzüge einer Allgemeinen Theorie der Linearen Integralgleichungen. Teubner.

Holway, L. H. 1963 Approximation procedures for kinetic theory. PhD, Harvard.

Holway, L. H. 1966 New statistical models for kinetic theory: methods of construction. Phys. Fluids 9 (9), 1658.

Homolle, T. M. M. \& Hadjiconstantinou, N. G. 2007 A low-variance deviational simulation Monte Carlo for the Boltzmann equation. J. Comput. Phys. 226 (2), 2341-2358.

Juvé, V., Crut, A., Maioli, P., Pellarin, M., Broyer, M., Del Fatti, N. \& Vallée, F. 2010 Probing elasticity at the nanoscale: terahertz acoustic vibration of small metal nanoparticles. Nano Lett. 0-5.

VON KÁrmán, T. 1963 From Low-speed Aerodynamics to Astronautics. Pergamon.

KNUdSEN, M. 1909a Die Gesetze der molekular Strömung und der inneren Reibungströmung der Gase durch Röhren. Ann. Phys. 28, 75-130.

Knudsen, M. $1909 b$ Eine Revision der Gleichgewichtsbedingung der Gase: Thermische Molekularströmung. Ann. Phys. 336 (1), 205-229.

Knudsen, M. 1910 Thermischer Molekulardruck der Gase in Röhren und porösen Körpern. Ann. Phys. 336 (3), 633-640.

Lagubeau, G., Le Merrer, M., Clanet, C. \& Quéré, D. 2011 Leidenfrost on a ratchet. Nat. Phys. 7 (5), 395-398. 
Lebowitz, J. L., Frisch, H. L. \& Helfand, E. 1960 Nonequilibrium distribution functions in a fluid. Phys. Fluids 3, 325-338.

LoyAlKA, S. K. 1971 Kinetic theory of thermal transpiration and mechanocaloric effect. Part 1. J. Chem. Phys. 55 (9), 4497.

Loyalka, S. K., Petrellis, N. \& Storvick, T. S. 1979 Some exact numerical results for the BGK model: Couette, Poiseuille and thermal creep flow between parallel plates. Z. Angew. Math. Phys. 30 (3), 514-521.

LOYALKA, S. K. \& TOMPSON, R. V. 2009 The velocity slip problem: accurate solutions of the BGK model integral equation. Eur. J. Mech. B 28 (2), 211-213.

Manela, A. \& Hadjiconstantinou, N. G. 2007 On the motion induced in a gas confined in a small-scale gap due to instantaneous boundary heating. J. Fluid Mech. 593, 453-462.

MaXwell, J. C. 1879 On stresses in rarified gases arising from inequalities of temperature. Phil. Trans. R. Soc. Lond. 170, 231-256.

NAssios, J. \& SADER, J. E. 2012 Asymptotic analysis of the Boltzmann-BGK equation for oscillatory flows. J. Fluid Mech. 708 (1960), 197-249.

OHWADA, T. \& Sone, Y. 1992 Analysis of thermal stress slip flow and negative thermophoresis using the Boltzmann equation for hard-sphere molecules. Eur. J. Mech. B 11 (4), 389-414.

OHwadA, T., Sone, Y. \& AOKI, K. 1989 Numerical analysis of the shear and thermal creep flows of a rarefied gas over a plane wall on the basis of the linearized Boltzmann equation for hard-sphere molecules. Phys. Fluids A 1, 1588.

PARK, J. H., BAhukudumbi, P. \& BeskoK, A. 2004 Rarefaction effects on shear driven oscillatory gas flows: a direct simulation Monte Carlo study in the entire Knudsen regime. Phys. Fluids 16 (2), 317.

Pelton, M., Sader, J. E., Burgin, J., Liu, M., Guyot-Sionnest, P. \& Gosztola, D. 2009 Damping of acoustic vibrations in gold nanoparticles. Nat. Nanotechnology 4 (8), $492-495$.

Preziosi, L. 1992 Thermal creep problems by the discrete Boltzmann equation. Transp. Theory Stat. Phys. 21 (3), 183-209.

Radtke, G. A., Hadjiconstantinou, N. G. \& Wagner, W. 2011 Low-noise Monte Carlo simulation of the variable hard sphere gas. Phys. Fluids 23 (3), 030606.

Ramanathan, S. \& Koch, D. L. 2009 An efficient direct simulation Monte Carlo method for low Mach number noncontinuum gas flows based on the Bhatnagar-Gross-Krook model. Phys. Fluids 21 (3), 033103.

SHARIPOV, F. \& KALEMPA, D. 2007 Gas flow near a plate oscillating longitudinally with an arbitrary frequency. Phys. Fluids 19 (1), 017110.

ShARIPOV, F. \& KALEMPA, D. 2008 Oscillatory Couette flow at arbitrary oscillation frequency over the whole range of the Knudsen number. Microfluid Nanofluid 4 (5), 363-374.

ShI, Y. \& SADER, J. E. 2010 Lattice Boltzmann method for oscillatory Stokes flow with applications to micro- and nanodevices. Phys. Rev. E 81 (3), 1-14.

Sone, Y. 1966 Thermal creep in rarefied gas. J. Phys. Soc. Japan 21, 1836-1837.

SonE, Y. 1969 Asymptotic theory of flow of rarefied gas over a smooth boundary I. In Rarefied Gas Dynamics (ed. L. Trilling \& H. Y. Wachman), p. 243. Academic.

Sone, Y. 1972 A flow induced by thermal stress in rarefied gas. J. Phys. Soc. Japan 33, $232-236$.

SONE, Y. 1974 Asymptotic theory of flow of rarefied gas over a smooth boundary. Part 2. Trans. Japan. Soc. Aeronaut. Space Sci. 17, 113-122.

SONE, Y. 1984 Highly rarefied gas around a group of bodies with various temperature distributions. Part 1. Small temperature variation. J. Méc. Théor. Appl. 3 (2), 315-328.

Sone, Y. 1985 Boundary temperature effect in a highly rarefied gas. Phys. Fluids 28 (1), 419.

Sone, Y. 2000 Kinetic Theory and Fluid Dynamics. Birkhäuser.

Sone, Y. 2007 Molecular Gas Dynamics: Theory, Techniques, and Applications. Springer.

Sone, Y., OHWADA, T. \& AOKI, K. 1989 Temperature jump and Knudsen layer in a rarefied gas over a plane wall: numerical analysis of the linearized Boltzmann equation for hard-sphere molecules. Phys. Fluids A 1 (2), 363-370.

Struchtrup, H. 1997 The BGK-model with velocity-dependent collision frequency. Contin. Mech. Thermodyn. 9 (1), 23-31. 
Struchtrup, H. \& TORRILhon, M. 2003 Regularization of Grad's 13 moment equations: derivation and linear analysis. Phys. Fluids 15 (9), 2668.

TAheri, P., Rana, A. S., Torrilhon, M. \& Struchtrup, H. 2009 Macroscopic description of steady and unsteady rarefaction effects in boundary value problems of gas dynamics. Contin. Mech. Thermodyn. 21 (6), 423-443.

TAKATA, S. \& HATTORI, M. 2012 Asymptotic theory for the time-dependent behavior of a slightly rarefied gas over a smooth solid boundary. J. Stat. Phys. 147 (6), 1182-1215.

Tamada, K. \& Sone, Y. 1966 Some studies on rarefied gas flows. J. Phys. Soc. Japan 21 (7), $1439-1445$.

TAng, G., Gu, X.-J., BArber, R., Emerson, D. R. \& Zhang, Y. 2008 Lattice Boltzmann simulation of nonequilibrium effects in oscillatory gas flow. Phys. Rev. E 78 (2), 1-8.

TORRILHON, M. \& StRUChTRUP, H. 2008 Boundary conditions for regularized 13-momentequations for micro-channel-flows. J. Comput. Phys. 227 (3), 1982-2011.

ToscAni, G. 2009 Wealth redistribution in conservative linear kinetic models. Europhys. Lett. 88 (1), 10007.

Vargo, S. E., Muntz, E. P., Shiflett, G. R. \& TAng, W. C. 1999 Knudsen compressor as a micro- and macroscale vacuum pump without moving parts or fluids. J. Vac. Sci. Technol. A 17 (4), 2308.

Vincenti, W. G. \& Kruger, C. H. Jr 1965 Introduction to Physical Gas Dynamics, 8th edn. Krieger.

Wagner, W. 2008 Deviational particle Monte Carlo for the Boltzmann equation. Monte Carlo Meth. Applic. 14 (3), 191-268.

Welander, P. 1954 On the temperature jump in a rarefied gas. Ark. Fys. 7, 507-553.

WÜrger, A. 2011 Leidenfrost gas ratchets driven by thermal creep. Phys. Rev. Lett. 107 (16), 1-4.

YAP, Y. W. \& SADER, J. E. 2012 High accuracy numerical solutions of the Boltzmann Bhatnagar-Gross-Krook equation for steady and oscillatory Couette flows. Phys. Fluids 24 (3), 032004.

Yu, H., GiRimaji, S. \& LUO, L.-S. 2005 Lattice Boltzmann simulations of decaying homogeneous isotropic turbulence. Phys. Rev. E 71 (1), 1-5. 